\title{
Individual versus family cognitive behavioural therapy in children with anxiety disorders. A clinical and economic evaluation
}

Citation for published version (APA):

Bodden, D. H. M. (2006). Individual versus family cognitive behavioural therapy in children with anxiety disorders. A clinical and economic evaluation. [Doctoral Thesis, Maastricht University]. Datawyse / Universitaire Pers Maastricht. https://doi.org/10.26481/dis.20070216db

Document status and date:

Published: 01/01/2006

DOI:

10.26481/dis.20070216db

Document Version:

Publisher's PDF, also known as Version of record

Please check the document version of this publication:

- A submitted manuscript is the version of the article upon submission and before peer-review. There can be important differences between the submitted version and the official published version of record.

People interested in the research are advised to contact the author for the final version of the publication, or visit the DOI to the publisher's website.

- The final author version and the galley proof are versions of the publication after peer review.

- The final published version features the final layout of the paper including the volume, issue and page numbers.

Link to publication

\footnotetext{
General rights rights.

- You may freely distribute the URL identifying the publication in the public portal. please follow below link for the End User Agreement:

www.umlib.nl/taverne-license

Take down policy

If you believe that this document breaches copyright please contact us at:

repository@maastrichtuniversity.nl

providing details and we will investigate your claim.
}

Copyright and moral rights for the publications made accessible in the public portal are retained by the authors and/or other copyright owners and it is a condition of accessing publications that users recognise and abide by the legal requirements associated with these

- Users may download and print one copy of any publication from the public portal for the purpose of private study or research.

- You may not further distribute the material or use it for any profit-making activity or commercial gain

If the publication is distributed under the terms of Article $25 \mathrm{fa}$ of the Dutch Copyright Act, indicated by the "Taverne" license above, 


\section{Individual versus Family \\ Cognitive Behavioural Therapy in children with anxiety disorders}

A clinical and economic evaluation 
Cover design: Corien Bögels

Print: Datawyse

ISBN 978-90-5278-598-1

(c) D.H.M Bodden, Maastricht 2006 


\section{Individual versus Family Cognitive Behavioural Therapy in children with anxiety disorders}

\section{A clinical and economic evaluation}

\section{PROEFSCHRIFT}

ter verkriiging van de graad van doctor aan de Universiteit Maastricht, op gezag van Rector Magnificus, Prof. Mr. G.P.M.F. Mols, volgens het besluit van het College van decanen, in het openbaar te verdedigen op vrijdag 16 februari 2007 om 14.00 uur

door

Denise Hubertina Michiel Bodden

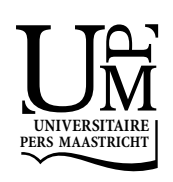


Promotor:

Prof. dr. S. M. Bögels, Universiteit van Amsterdam

Copromotor:

Dr. C. D. Dirksen

Beoordelingscommissie:

Prof. dr. A. Arntz (voorzitter)

Prof. dr. F. de Boer, Universiteit van Amsterdam

Prof. dr. C. Braet, Universiteit Gent

Prof. dr. A. Jansen

Prof. dr. H. Severens

This study was supported by grant 945-02-052 from ZONMW, the Netherlands. 
Angst door Beaudy (8 jaar)

alle mensen hebben weleens een angst. soms voor een spin of voor een hond. deze angsten komen ergens vandaan?

dit kan zijn door nare dingen bv: auto ongeluk of een televiesie progamma of net als bij mij dat ze mijn been opnieuw hebben moeten breken zonder verdoving en daar heb ik nu ook een angst

van over gehouden bv: angst dat ze mijn pijn gaan doen.

soms kunnen mensen of kinderen ook bang zijn als ze iets vreemds horen en dat geluid niet kennen.

ook hebben veel kinderen de angst dat hun vader of moeder dood of weggaan. in heel veel gevallen kun je hier voor naar een praatdokter gaan daar proberen ze met je te praten over jou angsten.

in de hoop dat je naar een paar gesprekjes anders naar die angst gaat kijken en denken.

bv: ik was in het begin ook heel bang voor het ziekenhuis en dokters.

maar nu naar bijna een half jaar verder en elke week naar de praatdokter ben geweest zijn mijn angsten bijna weg. 
This thesis is dedicated to my grandma, Jenny Magnéé 


\section{Table of Contents}

Chapter 1. Introduction 9

Chapter 2. Societal burden of clinically anxious youth referred

for treatment: a cost-of-illness study

Chapter 3. The diagnostic utility of the Screen for Child Anxiety Related Emotional Disorders-71 (SCARED-71)

Chapter 4. Negative automatic thinking in clinically anxious and control youth: The effects of gender, age and content-specificity

Chapter 5. Cognitive Behavioural Therapy in children with anxiety disorders; an overview

Chapter 6. Efficacy of Individual versus Family Cognitive

Behavioural Therapy in Clinically Anxious Youth

Chapter 7. Costs and cost-effectiveness of family CBT versus individual CBT in clinically anxious children

Chapter 8. Discussion

References

Samenvatting

Abstract

Dankwoord

Curriculum Vitae 

Chapter 1

\section{Introduction}


Children experience many fears over the course of their development. These fears are normal and will disappear spontaneously. However, in some children these fears become persistent and intervene with daily life, which causes normal fears to become pathological fears or anxiety disorders. Anxiety disorders are often associated with social problems, school problems, low selfesteem and an increased risk for other mental disorders such as depression and substance abuse (Kendall, Safford, Flannery-Schroeder, \& Webb, 2004; Strauss, Frame, \& Forehand, 1987; Strauss, Last, Hersen, \& Kazdin, 1988). Evenmore, anxiety disorders are one of the most prevalent mental disorders worldwide. International prevalence rates for anxiety disorders in children range from $1.8 \%$ (Anderson, Williams, McGee, \& Silva, 1987) to 25\% (Kessler et al., 1994). However, when interference with daily functioning is taken into account these prevalence rates reduce to 5 to $10 \%$ of the children (Klein \& Pine, 2002).

Not only are anxiety disorders an enormous problem with respect to magnitude but also with respect to the costs associated with anxiety disorders and their consequences. Costs of anxiety disorders were estimated to be 63.1 billion dollars in the USA in 1998 by extrapolating the costs of 1990 (Greenberg et al., 1999). In the Netherlands, health care costs of anxiety disorders across all ages were 180 million euro in 1999, accounting for $0.5 \%$ of the health care budget. More specifically, health care costs due to anxiety disorders in children and young adults aged 1 to 24 amounted to 21.6 million euros using a top-down method from existing national registrations (Polder, Takken, Meerding, Kommer, \& Stokx, 2002). However, bottom-up or patient reported costs of anxiety disorders in children have not yet been examined. In chapter 2 of this thesis, the societal costs of clinically anxious children and adolescents referred for treatment will be investigated.

There is a need for an effective treatment that could decrease the costs associated with anxiety disorders. In order to determine the effectiveness of a certain therapy, reliable and valid methods are necessary to measure the change due to treatment. Besides diagnostic interviews, questionnaires can be used to measure treatment effects. For instance anxiety questionnaires such as the Screen for Child Anxiety Related Emotional Disorders Revised (SCARED-R; Muris, Merckelbach, Schmidt, \& Mayer, 1999) can be used to detect differences in anxiety symptoms and to calculate the magnitude of change, the so-called effect sizes. The SCARED-R differentiates from other child anxiety questionnaires because it taps into all DSM-IV (APA, 1994) childhood anxiety disorders and consists of a child and parent version. Cognitions questionnaires like the Children's Automatic Thought Scale (CATS; Schniering \& Rapee, 2002) can be used to determine if a certain treatment is effective in changing cognitions of anxious children. The CATS has the advantage of being a developmentally sensitive cognition questionnaire for children measuring negative automatic thoughts across both internalizing and externalizing problems in children and adolescents. However, the SCARED was altered into a 71-version by adding 5 social phobia items because the 66-item SCARED-R did 
not sufficiently cover social phobia. The CATS was translated into a Dutch version. However, since the SCARED and CATS questionnaire were adapted, no psychometric properties were available. Therefore, a study was conducted to differentiate children with anxiety disorders from children from the general population with respect to their anxiety symptoms (SCARED-71) and their anxious cognitions (CATS). The psychometric properties of the SCARED-71 and the CATS will be examined in respectively chapter 3 and 4 of this thesis.

Anxiety disorders are likely to continue into adulthood, however the type of anxiety disorder and the severity of the disorder vary over time (Craske, 1997). If untreated the prognosis of anxiety disorders is quite poor (Keller et al., 1992). Several therapies have been applied to children with anxiety disorders e.g. pharmacotherapy, psychodynamic therapy, Eye movement Desensitisation and Reprocessing (EMDR) and relaxation therapy. However, Cognitive Behavioural Therapy (CBT) is the only evidence-based effective treatment in children with anxiety disorders. Kendall was the first to develop a CBT program for children with anxiety disorders namely Coping cat (Kendall, Kane, Howard, \& Siqueland, 1990). It was shown that $64 \%$ of the children was free of their primary diagnosis after treatment (Kendall, 1994). Since then a lot of variations of this CBT program, for instance group CBT, have been developed.

Whether or not to involve parents in CBT is another issue that has been raised during the last years. Barrett, Dadds, and Rapee (1996) found that individual CBT with family management was more effective than individual CBT alone at post treatment and 1-year follow-up (Barrett et al., 1996). This was especially the case, if parents were anxious themselves (Cobham et al. 1998). However, four recent studies and a meta-analysis did not show an additive value of parental involvement (In-Albon \& Schneider, in press; Nauta, Scholing, Emmelkamp, \& Minderaa, 2001 and 2003; Siqueland, Rynn, \& Diamond, 2005; Wood, Piacentini, Southam-Gerow, Chu, \& Sigman, 2006). Chapter 5 of this thesis will provide an overview of CBT during the last quarter of this century.

Recently a new family based CBT was developed, which includes both parents and siblings of anxious children (Bögels \& Siqueland, 2006). In this 12session family CBT, parents, siblings and the anxious child are seen simultaneously. Child and parents are taught to reduce their own anxiety using CBT skills. Furthermore, parents learn to model, guide and reward the exposure of the child and dysfunctional thoughts and problematic family interaction are being modified. A pilot study of 17 families showed that $59 \%$ of the children were free of their primary anxiety disorder at 3-month follow-up (Bögels, \& Siqueland, 2006). This pilot study was extended into a randomized control trial. In chapter 6 , the effectiveness of this family CBT in comparison to an individual or child based CBT in 128 clinically anxious children and their families will be examined.

In order to investigate whether family CBT or individual CBT is more cost-effective, a cost-effectiveness study was conducted. A cost-effectiveness study evaluates and compares the difference in costs related to the difference in 
effectiveness between two treatments with the purpose of improving resource allocation decisions by policy makers and insurers, and consequently, to improve individual, societal and economical wellbeing (Knapp, 1997). This costeffectiveness study evaluated and compared the difference in costs and outcomes of individual and family CBT. To date, no studies have addressed the costs associated with CBT in children with anxiety disorders and subsequently, its cost-effectiveness. The results of the cost-effectiveness analyses will be presented in chapter 7 . In the last chapter, a short overview and the clinical implications of these five studies are discussed. Future research suggestions and concluding remarks will be given. 


\section{Chapter}

\section{Societal burden of clinically anxious youth referred for treatment: a cost-of-illness study}

Denise H.M. Bodden, Carmen D. Dirksen, \& Susan M. Bögels 


\begin{abstract}
Objective: To investigate cost-of-illness in clinically anxious youth aged 8-18 in the Netherlands and the discriminative and convergent validity of the cost diary used.

Method: A prevalence-based cost-of-illness study using a societal perspective was conducted. Discriminant validity was obtained by comparing costs of families with an anxious child $(n=118)$ to costs of families from the general population $(n=41)$. Bottom-up costs were derived from cost diaries, while topdown costs were obtained from national registrations.

Results: Costs using a bottom-up approach amounted to $€ 2748$ per family of a clinically referred anxious child a year, which is more than 20 million euros a year for the Dutch population. Costs of families with clinically anxious children were almost 21 times as high compared to families from the general population. With respect to convergent validity, costs using the bottom-up approach from clinically anxious children were quite comparable compared to those of topdown data of anxious children.

Conclusion: Clinical anxiety disorders in childhood cost the Dutch society more than 20 million euros a year. Based on results of discriminate and convergent validity, the cost diary seems a valid method in establishing cost-of-illness in childhood anxiety disorders.
\end{abstract}

Keywords; anxiety disorders, cost-of-illness, children, cost diary 


\section{Introduction}

Anxiety disorders are the most common childhood mental disorders. Prevalence rates for children with anxiety disorders vary considerably in the international literature from 1.8 (Anderson, Williams, McGee, \& Silva, 1987) to $25 \%$ (Kessler et al., 1994). When impairment is taken into consideration, Klein and Pine (2000) estimated the prevalence rate to be between 5 to $10 \%$. The yearly prevalence rate of children with a mental disorder seeking treatment in mental health care ranges from 1.6\% (Sytema \& Koopmans, 1998) to 8.1\% (Leaf et al., 1996).

In the Netherlands, a six-month prevalence rate of $23.5 \%$ for anxiety disorders was found in 13- to 18-year-old adolescents. However, when impairment in daily functioning was taken into account the prevalence rate dropped to $9.7 \%$ (Verhulst, Van der Ende, Ferdinand, \& Kasius, 1997) Moreover, only $3.5 \%$ of the Dutch 4 - to 18-year-old children with a mental disorder had been referred yearly to mental health care for treatment (Verhulst \& Van der Ende, 1997).

With respect to costs, the total costs for anxiety disorders were highest of all mental illnesses, namely 46.6 billion dollars, accounting for $31.5 \%$ of total expenditures for mental health in 1990 in the USA (Dupont et al., 1996). Greenberg and colleagues (1999) estimated the annual cost of anxiety disorders to be 63.1 billion dollars in the USA in 1998 by extrapolating the costs of 1990 . Besides direct costs of treating anxiety disorders, also indirect costs due to impaired social functioning, such as financial dependence and unemployment are found in adults with anxiety disorders (Leon, Portera, \& Weissman, 1995). In the Netherlands, health care costs of anxiety disorders across all ages were 180 million euro in 1999 , accounting for $0.5 \%$ of the health care budget. More specifically, health care costs due to anxiety disorders in 1- to 24-year-old children and young adults amounted to 21.6 million euros (Polder et al., 2002).

An analysis to measure the magnitude of the costs related to illness is called a cost-of-illness study. A cost-of-illness study involves an estimation of the total economic burden, which an illness poses on society (Polder et al., 2002; Rice \& Miller, 1995). Essentially there are two approaches to establish the costof-illness, namely the bottom-up and the top-down approach.

The bottom-up approach acquires data on patient level by means of registrations or self-report measures. Self-report measures contain retrospective cost questionnaires, retrospective cost interviews or prospective cost diaries (Bruijnzeels et al., 1998a; Sleed, Eccleston, Beecham, Knapp, \& Jordan, 2005). The bottom-up approach enables one to capture resource use that is likely to vary from patient to patient and may not be easily extracted from existing data sources. Multiplying the costs per patient by the national prevalence rate results in the total cost-of-illness for a particular patient group. An advantage of the bottom-up approach is that detailed data can be obtained including non-health related costs, such as costs due to productivity losses and out-of-pocket costs. A 
disadvantage of this method may be that the sample of patients used for the cost-of-illness calculations is not representative for the patient group, for example due to strict inclusion and exclusion criteria (Oostenbrink, Koopmanschap, \& Rutten, 2005; Polder et al., 2002).

The top-down approach gathers data on patient group level from existing (national) registrations. The total costs per health care sector, for example hospital care, are considered to be the basis from which costs can be deducted per patient group. An advantage of this approach is that there are no problems with co-morbidity, since only the primary diagnosis is taken into account. A disadvantage is that registrations may be incomplete and contain only global data, which makes it difficult to provide a detailed specification of the costs per patient group. Furthermore, when using a top-down approach it is difficult if not impossible to obtain costs outside the health care sector (Oostenbrink et al., 2005; Polder et al., 2002).

Another important distinction in cost-of-illness studies is that costs can be determined from a prevalence-based or incidence-based perspective. In a prevalence-based approach, the costs of a disorder are determined for a fixed period of time, as a result of the prevalence of the disorder (number of cases of a disorder at a specific time). This period is called base period and most often studies investigate the annual economic burden, thus based on a year (Ament \& Evers, 1993; Rice \& Miller, 1995). A disadvantage of the prevalence-based approach is that it may be difficult to isolate potential differences in costs by stage or duration of the disorder (Greenberg et al., 1999). The incidence based approach estimates the lifetime costs of an illness from its onset, thus costs are assigned to the year in which the illness sets off (Ament \& Evers, 1993; Rice \& Miller, 1995). Incidence based studies can isolate the differences in costs by stage or duration of the disease. However, a disadvantage of using this approach is that follow up research is required.

In this article, a prevalence-based cost-of-illness analysis was performed in 8- to 18-year-old children and adolescents with anxiety disorders, who were referred to treatment. The primary aim of this study was to establish the societal costs of illness in families with a clinically anxious child, using the bottom-up approach by means of a prospective cost diary. A secondary aim of this study was to investigate the discriminative validity of the prospective cost diary by comparing the costs related to anxiety, psychological, physical and other problems in families with a clinically anxious child to the same costs in families from the general population. The third aim of this study was to establish convergent validity by comparing bottom-up acquired health care costs obtained with the cost diaries of the clinically anxious children with topdown acquired health care costs of children with a primary diagnosis of anxiety disorder. 


\section{Method}

\section{Participants}

For the cost-of-illness study and the discriminative validity study, participants were clinically anxious children and children from the general population, respectively. The clinically anxious group consisted of 118 families with a child referred for community mental health treatment because of anxiety, and participated in a multi-centre randomized clinical trial comparing family CBT with individual CBT (Bodden et al., submitted). This study was conducted from March 2002 to November 2005, inclusion ended in March 2004. Inclusion criteria were (1) age 8-18, (2) a primary anxiety disorder (except for obsessivecompulsive and post-traumatic stress disorder), (3) IQ $\geq 80$, (4) at least one parent willing to participate. Children were excluded if suffering from (1) substance abuse, (2) current suicide attempts, (2) psychoses, (3) autismspectrum disorders, and (4) untreated Attention Deficit Hyperactivity Disorder (ADHD). Children using medication for their anxiety were only included if dosage was kept constant during treatment and follow-up.

There were 74 girls $(63 \%)$ and 44 boys $(37 \%)$, range 8 -17 years $(M=12.4$, $S D=2.6)$. Most were Caucasian $(n=117,99 \%)$ and $53(45 \%)$ attended primary education. The remaining $65(55 \%)$ attended secondary education. The mean number of diagnosis per child was $2.9(S D=1.7)$, whereas the mean severity level of the primary diagnosis was $7.1(S D=1.0)$. According to the compound diagnosis on the Anxiety Disorder Interview Schedule (ADIS; Silverman \& Nelles, 1988) their primary anxiety diagnosis was social phobia $(n=37,31 \%)$, separation anxiety $(n=32,27 \%)$, generalized anxiety disorder $(n=21,18 \%)$, specific phobia $(n=20,17 \%)$, and panic disorder with or without agoraphobia $(n=8,7 \%)$. Of the 118 children, $94(80 \%)$ had one or more co morbid anxiety disorder(s); separation anxiety disorder $(n=20,17 \%)$, social phobia $(n=43,36 \%)$, specific phobia $(n=51,43 \%)$, panic disorder $(n=20,17 \%)$, generalized anxiety disorder $(n=42,36 \%)$, obsessive-compulsive disorder $(n=5,4 \%)$, and posttraumatic stress disorder $(n=7,6 \%)$. Moreover, $29(25 \%)$ were comorbid with dysthymic disorder or depression, 9 (8\%) with ADHD, 2 (2\%) with conduct disorder, and $1(1 \%)$ with oppositional defiant disorder.

The sample from the general population consisted of a group of 41 families who were recruited via advertisements. Children of these families were comparable to children from the clinically anxious group based on age, gender and educational level. There were 27 girls (66\%) and 14 boys (34\%), range 8-17 years $(M=12.5, S D=2.5)$. Most were Caucasian $(n=40,98 \%)$ and $16(39 \%)$ attended primary school. The remaining $25(61 \%)$ attended secondary education. According to the compound diagnosis on the ADIS, the prevalence of anxiety disorders in this sample was $12 \%(n=5)$ and $2.4 \%(n=1)$ for ADHD, closely resembling Dutch prevalence of anxiety disorders and ADHD, respectively $9.7 \%$ and $2.3 \%$ (Verhulst et al., 1997). The primary anxiety 
diagnoses consisted of social phobia $(n=1,20 \%)$ and specific phobia $(n=4,80 \%)$ with a mean severity level of $4.8(S D=0.8)$. The overrepresentation of simple phobia and the relatively low severity rates of the anxiety disorders in the control group also support the "normality" of the control children.

All children and parents received and signed an informed consent. The ADIS child and parent version were administered to obtain diagnostic status. Clinically anxious children, their siblings and parents were assessed before treatment and families from the general population were only measured once. Furthermore, all family members had to fill in a battery of questionnaires, among which a prospective cost diary.

\section{Prospective cost diary}

A cost diary was used to determine the resources used (Freer, 1980; Goossens, Rutten-van Mölken, Vlaeyen, \& Van der Linden, 2000) in both the cost-of-illness study as in the discriminative validity study. Cost dairies were completed prospectively by one of the parents and covered a period of two weeks. Families of clinically anxious children received the cost diary 2 weeks before treatment and the sample of families from the general population received the same cost diary during their one time assessment. Each cost diary was presented in a booklet form with instructions and an example diary. Parents recorded the following resource use: visits to psychologist or psychotherapist, visits to the general practitioner, outpatient consultations at the hospital, medication, day treatment, costs of paid house help, children's day care, after school care, informal care, productivity losses due to absence of work by the parents, loss of household activities or voluntary work, loss of leisure time, absence from school of the child, distance from health care facilities and out-ofpocket expenses, such as transportation costs and monetary contributions. Also, parents recorded the name of the family member for whom the resource use was applicable (child, sibling, mother or father), as well as the reason for the resource use by that family member. For analysis, the reported reasons were grouped into (1) due to anxiety, (2) due to psychological problems, (3) due to physical problems, and (4) due to other reasons.

\section{Cost prices}

Costs were calculated by multiplying the resources used, obtained with the prospective cost diaries, by the cost price of each resource. Costs were calculated at 2003 euros $\left(1 €=0.087 \$\right.$, July $\left.30^{\text {th }} 2003\right)$. Almost all cost prices were obtained by using published Dutch guideline prices (Oostenbrink et al., 2005). However, medication prices were obtained from the Medication and aid Information Project database on the Internet (Board of Health Insurances, 2005) and were based on the Daily Defined Dosage (DDD), which indicates the mean medication usage per person a day with claw back and VAT (20\%). An average cost price was calculated for medication for anxiety problems and medication for psychological problems. 
As actual cost prices for day treatment and institutionalization were not available, the cost prices were derived from the average of actual reimbursements (Board Tariff rates Health care and Care authority, 2005). The cost price of day care was estimated by using a shadow price based on the actual day care costs for children under the age of 4 , derived from the Internet (Baby fun, 2005). To determine the costs associated with absence from school, actual annual tuition (National Institute of Budget information, 2005) was divided by the total annual hours at school, resulting in a cost price per hour of school absence. Productivity costs of the parents were calculated by means of the friction cost method, based on a mean added value of the Dutch working population (Oostenbrink et al., 2005). This method only takes into account production losses confined to the period needed (usually 90 days) to replace a sick employee.

\section{Cost-of-illness}

The primary aim of this study was to calculate the societal burden of illness of families of clinically anxious youth in The Netherlands. The cost-of-illness study was performed from a societal perspective and included direct health care costs, direct non-health care costs, indirect costs and out-of-pocket costs.

The costs per family over a period of two weeks were extrapolated to a period of 1 year (i.e. multiplied with 26) under the assumption that data obtained with the cost diaries were representative for that period (Goossens et al., 2000). Subsequently, the annual costs per family were multiplied with the prevalence figure of $9.7 \%$ (Verhulst et al., 1997) and the percentage of referred children of $3.5 \%$ (Verhulst et al., 1997), which results in a multiplication factor of $0.34 \%$, to calculate the total annual societal cost-of-illness of families of clinically anxious children referred for treatment. It was assumed that the percentage of referred children with a mental disorder $(3.5 \%)$ could be attributed to anxiety disorders as well. Furthermore, a low and high estimation of the cost-of-illness was calculated using the reported range of prevalence rates for children with anxiety disorders considering impairment and the reported range of percentages of children seeking treatment. As a result, a low multiplication factor of $0.08 \%$ (i.e. $1.6 \%$ of $5 \%$ ) and a high multiplication factor of $0.81 \%$ (i.e. $8.1 \%$ of $10 \%$ ) were used to calculate a low and high estimate of the societal cost-of-illness.

\section{Discriminative validity}

To determine the discriminative validity of the prospective cost diary, the societal costs of the 118 families of anxious children were compared to the societal costs of 41 families from the general population. For this purpose, all costs related to the child, irrespective of reason, were taken into account (i.e. psychological, physical or other). For both groups, the costs per family over a period of two weeks were extrapolated to a period of 1 year to obtain the annual costs per child for both groups. It was hypothesized that costs for 
anxiety problems were higher in the clinically anxious group compared to the general population but comparable between the two groups for psychological, physical and other problems.

\section{Convergent validity}

To establish convergent validity, health care costs per child per year obtained with the prospective cost diaries of 118 clinically anxious children were compared with the health care costs of children with a primary diagnosis of anxiety disorder, according to top-down registrations. For this comparison, only health-care costs due to anxiety of the child were considered, because the costs obtained with the top-down registration applied to children with a primary diagnosis of anxiety disorder and only consisted of costs related to the health care sector.

The top-down data were obtained from a generic cost study based on national registrations (Polder et al., 2002). In this study, the total costs of health care in 1999 were assigned to diagnosis groups based on actual delivered care, according to distribution ratios. This was done for each of the 21 health care sectors, among which mental health care. Diagnosis groups were based on the International Statistical Classification of Diseases, injuries and causes of death (ICD-9; World Health Organization, 1977). The ICD-9 is divided into 17 chapters, including chapter $\mathrm{V}$, mental and behavioural disorders. Furthermore, a more intricate classification is used, by itemizing the chapters into 96 specific diagnosis groups, including anxiety disorders. All anxiety disorders are taken into account (ICD-9 300.0-3 and 300.5-9) (World Health Organization, 1977).

The top-down data could not be compared directly to the group of 8 to 18 year old children included in the bottom-up cost-of-illness analysis, because costs were presented for the age groups 5- to 9, 10- to 14 and 15- to 19-year-olds. Therefore, the assumption was made that costs were equally distributed within the age groups, so the corresponding ages (8-18) could be filtered out by averaging the costs and multiplying them by each age year (Polder et al., 2002). Since top-down data were based on costs in 1999, for comparison with the bottom-up cost-of-illness approach, costs were actualized to 2003 euros by using the Consumer Price Index (CPI; Central office of statistics, 2005).

\section{Statistical analysis}

Statistical analysis could only be performed on the cost-of-illness study and on the discriminative validity study, since top-down data were not available on child level. Missing items in the cost diaries were handled with the Missing Value Analysis of SPSS based on the regression models using available data of the group mean, the available data of the individual and the pattern of change of the group. To investigate whether data were normally distributed, a Kolmogorov-Smirnov test was performed.

Due to highly skewed cost distributions, bootstrap simulations were conducted in order to get insight in significant differences on subtotal and total 
costs between families of clinically anxious children and families from the general population and the uncertainty surrounding these costs. The bootstrap method estimates the sampling distribution of a statistic through 1000 simulations, based on sampling with replacement from the original data (Briggs, Wonderling, \& Mooney, 1997). Bootstrap methods are increasingly being used for analysis of cost data, as traditional parametric and nonparametric statistical methods to analyse the difference in mean costs between groups, may be inappropriate when data are skewed (Barber \& Thompson, 2000; Desgagné, Castilloux, Angers, \& Lelorier, 1998).

\section{Results}

\section{Cost-of-illness}

All resources used and costs made related to the anxiety of the child by families with a clinically anxious child were summed and averaged for 2 weeks and one year (Table 1). The mean costs per family over 2 weeks were extrapolated to a year and amounted to $€ 2748$ per family with a clinically anxious child. Costs were not normally distributed, Kolmogorov-Smirnov $Z=4.5, p<0.01$, and skewed to the left, indicating that a minority of families reported high costs related to the anxiety of the child. The most prominent were costs related to institutionalized treatment ( $26 \%$ of total costs), day treatment $(23 \%$ of total costs), productivity loss of the parents ( $23 \%$ of total costs) and school absence (17\% of total costs).

The total number of Dutch children aged 8 to 18 in 2003 was computed using data of the Central Office of Statistics (2005), and resulted in 2,175,382 children. Multiplying this figure by $0.34 \%$ resulted in 7,385 Dutch children with an anxiety disorder that intervenes with daily functioning and who are referred to treatment. Hence, the annual societal cost-of-illness accumulated to $€ 2,748 \mathrm{x}$ $7,385=€ 20,293,958$ for families of clinically anxious children referred for treatment in the Netherlands. The same calculation was performed using the low and high multiplication factors. This resulted in a low estimate of the costof-illness of $€ 4,783,839$ and a high estimate of $€ 48,436,370$.

\section{Discriminative validity}

The mean total annual societal costs related to anxiety, psychological, physical and other reasons for families with a clinically anxious child and families from the general population are presented in Table 2 . In the clinically anxious group, costs due to anxiety reasons were highest ( $89 \%$ of total costs), followed by costs due to physical reasons ( $6 \%$ of total costs), costs due to other reasons (3\% of total costs) and psychological reasons ( $3 \%$ of total costs). The total annual costs of families with clinically anxious children due to anxiety problems, psychological problems, physical problems and other amounted to a mean of $€ 3,084(S D=8945)$ per child. 
Chapter 2

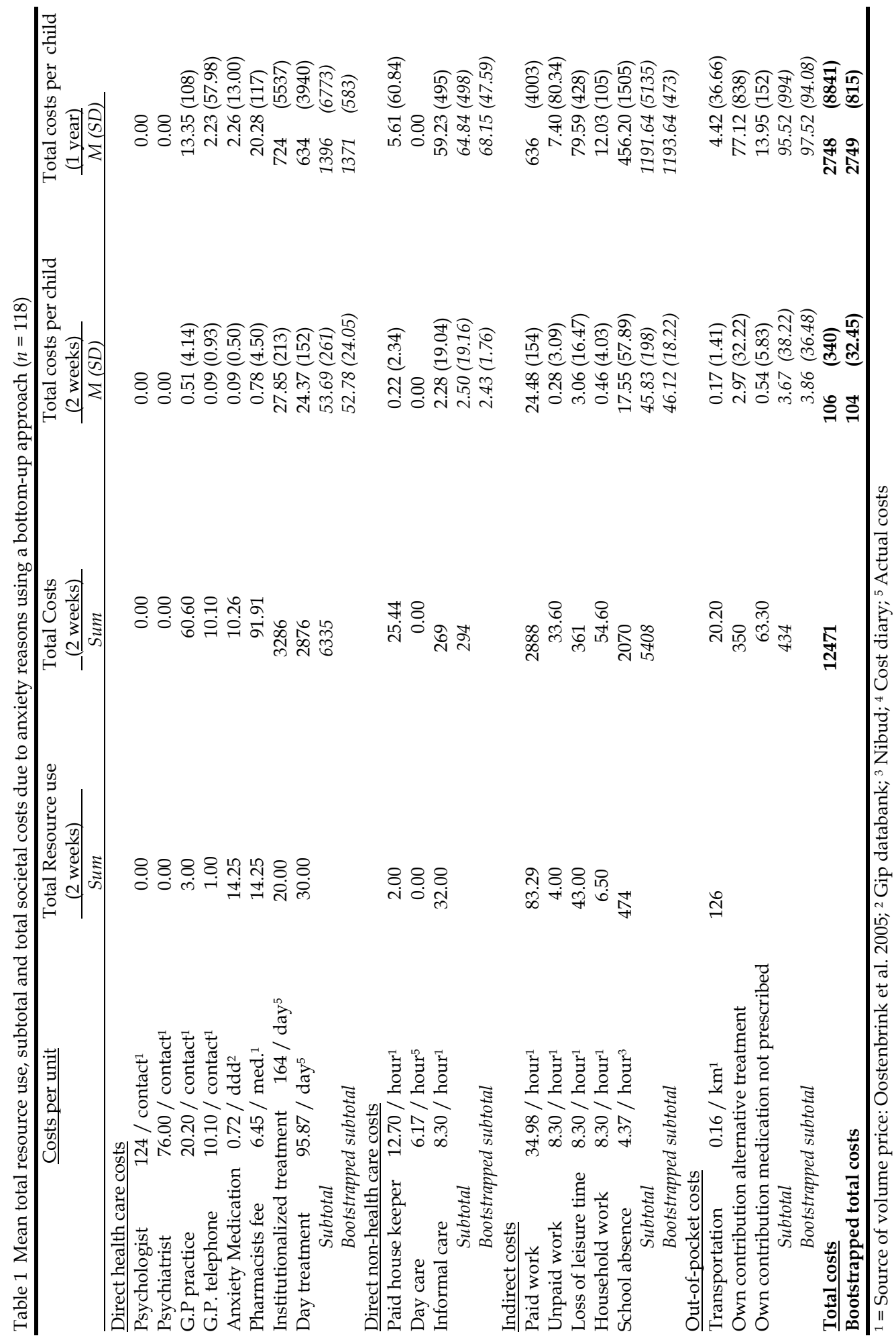


Societal burden of clinically anxious youth

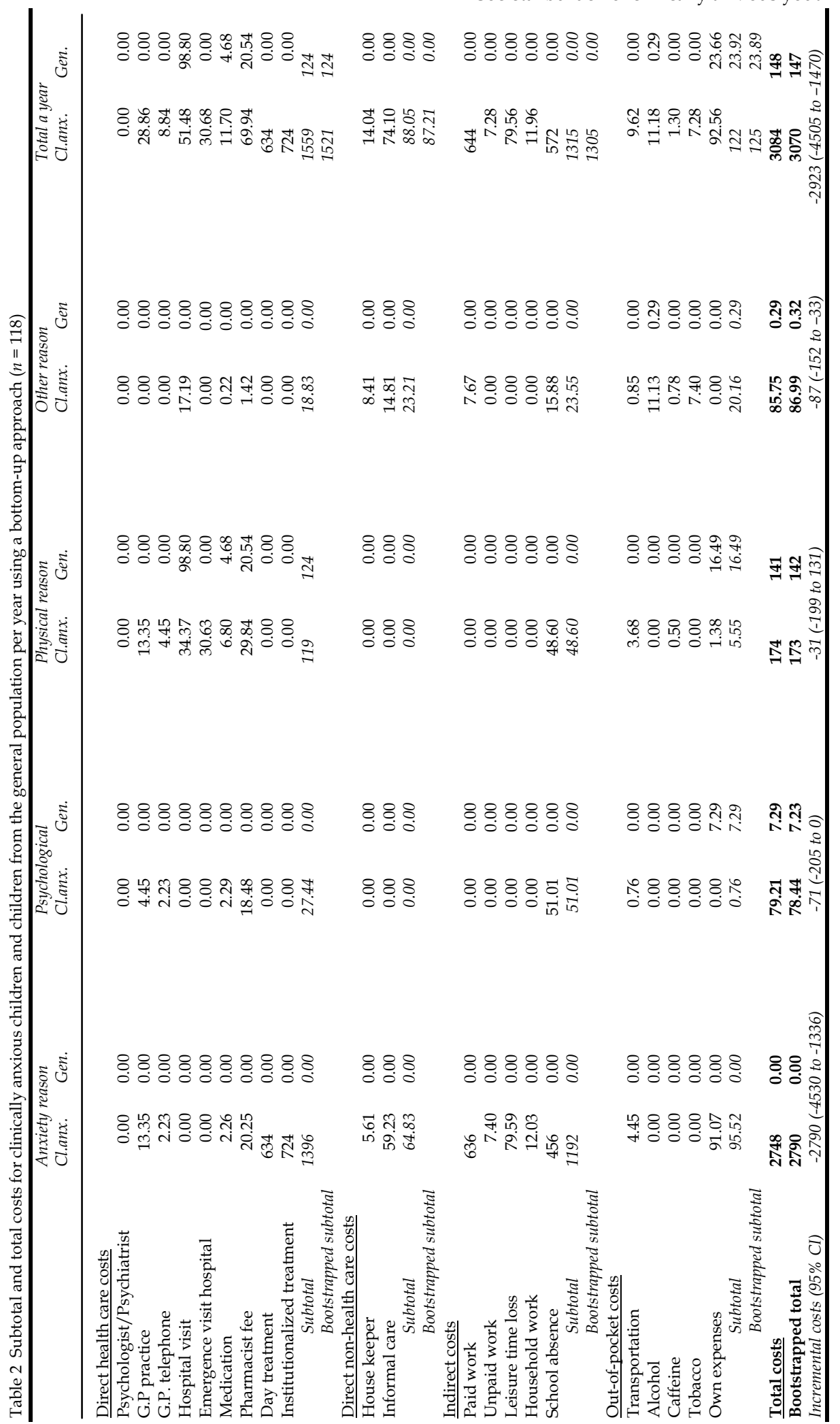


Costs of families from the general population were highest for physical reasons (95\% of total costs). A small proportion of costs was assigned to psychological reasons ( $5 \%$ of total costs) and other reasons ( $0.2 \%$ of total costs). Despite the fact that 5 children in the control group met criteria for an anxiety disorder, no costs were made concerning anxiety reasons. The total annual costs obtained from families of the general population amounted to a mean of $€ 148$ $(S D=523)$ per family.

Costs in both groups were not normally distributed (KolmogorovSmirnov $Z>1.6, p<0.05$ ). The bootstrapped results indicated that total societal costs were significantly higher in families with clinically anxious children compared to families from the general population. Subdividing the costs into reason showed significant higher costs related to anxiety and other reasons in families with a clinically anxious child compared to families from the general population. Costs related to psychological reasons were borderline significantly higher for the families with a clinically anxious child (see Table 2).

\section{Convergent validity}

The total health care costs of 8- to 18-year-old children with a primary diagnosis of anxiety disorder amounted to $€ 8,018,000$ in 1999 using the top-down approach, which augmented to $€ 9,075,613$ in 2003 when corrected with CPI. The total number of anxious 8- to 18-year-old children referred for treatment in 2003 was 7,385 (i.e. $2,175,382 \times 0.34 \%$ ). Dividing the total health care due to anxiety in children costs by this number resulted in $€ 1,229$ per anxious child that was referred (see Table 3).

Table 3 Direct health care costs, anxiety based per child a year based on the Bottom-up and top-down approach

\begin{tabular}{lcc} 
A day of hospitalization & 0.00 & 203 \\
Day treatment & 0.00 & 1.84 \\
Outpatient clinic & 0.00 & 42.51 \\
Categorical hospital & 0.00 & 32.71 \\
Remaining & 0.00 & 7.60 \\
$\quad$ Subtotal Hospital care & 0.00 & 287 \\
General practitioner & 15.58 & 58.27 \\
Paramedical care & 0.00 & 1.23 \\
$\quad$ Subtotal Primary care & 92.70 & 59.50 \\
Pharmaceutical help & 36.49 & 67.50 \\
Mental health care & 1358 & 785 \\
Management and care insurances & 0.00 & 29.92 \\
\multicolumn{1}{c}{ Total costs } & $\mathbf{1 4 1 0}$ & $\mathbf{1 2 2 9}$
\end{tabular}


Direct health care costs derived from the bottom-up method were used to compare with the top-down approach and amounted to $€ 1,410$ per referred anxious child (see Table 3 ). Total health care costs for clinically anxious children resulted in $€ 10,412,187$ with a low estimate of $€ 2,453,541$ and a high estimate of $€ 24,842,036$. Although the overall difference in total health care costs between the bottom-up and top-down approach per anxious child per year were about $€ 180$ (top-down 13\% lower than bottom-up), the differences in costs per health care sector were considerably higher. For instance, costs of medical hospital care were $€ 287$ higher using the top-down approach (23\% of total costs) compared to costs obtained with the bottom-up approach ( $0 \%$ of total costs). On the other hand, mental health care costs were $€ 573$ higher (top-down $42 \%$ lower than bottom-up) using the bottom-up approach ( $96 \%$ of total costs) compared to the costs obtained with the top-down approach (64\% of total costs).

\section{Discussion}

To the authors' knowledge, this is the first cost-of-illness study in clinically anxious children referred for treatment. The aim of this study was threefold. The first aim was to measure the societal costs of illness in clinically anxious children and their families, using a bottom-up approach by means of a prospective cost diary. The second aim was to investigate the discriminative validity of the prospective cost diary by comparing the costs of families with a clinically anxious child to the costs of families from the general population. The third aim of the study was to establish convergent validity by comparing bottom-up acquired health care costs of the clinically anxious children with topdown acquired health care costs of children with a primary diagnosis of anxiety.

With respect to results regarding cost-of-illness, bottom-up acquired total societal costs for families of clinically anxious children referred for treatment amounted to more than $€ 20$ million per year in the Netherlands with a low estimation of 4.8 and a high estimation of 48 million euros, which is a quite broad range. Costs amounted to $€ 2,748$ per family with a clinically anxious child per year. These costs seem relatively low compared to costs of other childhood psychopathology. Knapp, Scott and Davies (1999) conducted a pilot study on 10 children aged 4 to 10 with conduct disorders. Cost amounted to $€ 22,272$ per family per year. In another study on 11 children with autism spectrum disorders, societal costs were $€ 51,844$ per child per year (Järbrink, Fombonne, \& Knapp, 2003). However, it should be noticed that as these studies have low sample sizes, results should be considered with caution. Furthermore, the prevalence rate for anxiety is almost 4 times as high as conduct disorder (2,6\%, Verhulst et al., 1997) and 39 to 44 times higher as the prevalence rate for autism spectrum disorders $(0,22 \%$ to $0,25 \%$, van der Gaag, Robbroeckx, Smid, \& Verhulst, 1996). 
In line with Knapp et al. (1999) and Järbrink et al. (2003) a large part of the costs in our study can be attributed to the parents' productivity loss due to anxiety disorder of the child (23\% of total costs). Knapp et al. (1999) found that per family with a child with conduct disorder the annual costs due to lost employment were $€ 5,683$ (30\% of the total costs). Järbrink et al. (2003) showed that more than $50 \%$ of the total costs of children with an autism spectrum disorder were attributable to parents and costs related to income loss were $€ 17,405$ (34\% of total costs). Our study also found high costs due to school absenteeism (17\% of total costs). School absence can have long-term economic consequences such as decreased job opportunities or even unemployment. Taken together, both productivity costs of parents and school absence costs of the child seem very important and should be considered in future costing studies.

With respect to the results regarding discriminative validity, total costs of families with clinically anxious children using a bottom-up approach were almost 21 times as high compared to costs of families from the general population. In line with the hypothesis, the difference in costs between families of clinically anxious children and families from the general population can largely be subscribed to the significant difference in anxiety related costs, showing good discriminative validity of the cost diary on anxiety related costs. However, significant differences were also found on costs due to other reasons. In the prospective cost diary, the reasons for resource use were recorded based on subjective judgments by the parents. However, since anxiety is an internalizing disorder, parents may not always be able to interpret children's symptoms correctly. Therefore, they may have attributed costs mistakenly to non-anxiety reasons, e.g. a baby-sitter for a child old enough to stay on its own, if not anxious.

With respect to convergent validity, total direct health care costs related to anxiety were $13 \%$ lower using a top-down approach than using a bottom-up approach, which seems quite comparable. However, subdividing the costs into several cost categories showed that costs were distributed differently among the cost categories. For example, bottom-up acquired mental health care costs were $58 \%$ higher than top-down acquired mental health care costs. Furthermore, 23\% of the total costs using a top-down approach were attributed to hospital care, whereas no hospital care costs were found using the bottom-up approach. Hence, convergent validity on total direct health care costs were quite comparable, while the specific cost categories differed between bottom-up and the top-down approach. These findings suggest that children, who are not yet referred for mental health care, might consume more medical hospital care. Therefore, from a cost-of-illness perspective, it would also be interesting to investigate costs of families with children with anxiety disorders, who are not referred for mental health treatment, using the cost diary developed for this study. It might well be that these families make more medical health care costs, as the comparison with top-down acquired data suggests. 
This study has some limitations, which should be addressed. The first limitation is that the low and high multiplication factor used in this study caused a broad range of the societal costs of illness. This can be explained by the broad range of internationally reported prevalence rates and percentages of children seeking treatment due to differences in measurements, populations, etcetera. As a Dutch population was investigated, the most appropriate prevalence rate is $9.7 \%$ (Verhulst et al., 1997), which was obtained through the use of a reliable structured diagnostic interview. The same is true for the 3.5\% of children with mental disorders seeking treatment (Verhulst et al., 1997). Therefore, the prevalence and referral rates used in this study seem reliable.

The second limitation of the current study is that it can be argued that two-week cost diaries filled in by parents are not representative to reliably assess one-year bottom-up costs due to the limited time period. However, Goossens et al. (2000) have found that keeping a diary in a limited period namely one week every month, two weeks every two months or an entire year resulted in similar outcomes in fibromyalgia and pain patients. Another study of Bruijnzeels et al. (1998b) found that there was a substantial agreement between a 3-week cost diary and medical records concerning general practitioner visits of children, filled in by parents. Moreover, the inclusion period of this study lasted 2 years in which the cost diaries were filled out, therefore possible seasonal influences are ruled out.

The third limitation of this study is that although total health care costs obtained with the top-down and bottom-up method seemed quite comparable, the sample of children with a primary diagnosis of anxiety disorder used in the top-down study by Polder et al. (2002) might not be fully comparable to the clinically anxious children from our bottom-up approach in at least three respects. First, the children in the bottom-up sample were clinically anxious children with severe anxiety disorders. The mean number of anxiety disorders was 3 and the mean severity level 7, indicating severe interference with daily functioning. The children with a primary diagnosis of anxiety disorder in the top-down sample might suffer from different levels of anxiety disorders; some may have minor anxiety problems, while others may have severe anxiety problems. This kind of information was not available using the top-down data. Second, while the clinically anxious children in the bottom-up sample were awaiting CBT in a community mental health care setting, the anxious children in the top-down sample might be in different types and/or phases of treatment. Again, this kind of information was not available using the top-down data. Third, the clinically anxious children in the bottom-up sample were diagnosed using a semi-structured interview, the ADIS based on the DSM-IV (American Psychiatric Association, 1994). In the top-down sample, children were diagnosed by the doctor in attendance, who attributed the anxiety diagnosis to the patient based on the ICD-9 code, without a structured interview. Due to the classification method, the top-down estimation is possibly less reliable than the bottom-up cost estimation. 
In conclusion, this cost-of-illness study shows that the societal costs of families with a clinically anxious child who seek treatment amount to more than 20 million euros a year in the Dutch population, and were 21 times higher than in families of the general population. An effective treatment could decrease these costs on the long term. A recent cost-effectiveness study on family CBT versus individual CBT showed that societal costs in families with a clinically anxious child increased during treatment (due to total costs of 12 sessions CBT a €1612) but decreased directly after treatment to baseline level. Even more, during the 1-year follow-up societal costs dropped below the costs at baseline (Bodden et al., submitted). Since anxiety disorders tend to last into adulthood (Newman et al., 1996), societal costs are likely to be higher at the long term. This even results in anxiety disorders accounting for the highest costs of all mental illnesses in adulthood in the USA (Dupont et al., 1996). Hence, societal costs in adults with anxiety disorders can be saved by providing effective treatments to children with clinical anxiety disorders. 


\section{Chapter}

The diagnostic utility of the Screen for Child Anxiety Related Emotional Disorders-71 (SCARED-71)

Denise H.M. Bodden, Susan M. Bögels, \& Peter Muris 


\begin{abstract}
Objective: This study investigated the diagnostic utility of the Screen for Child Anxiety and Related Emotional Disorders (SCARED-71), as a screening tool for identifying anxiety disorders in youth.

Method: The SCARED-71 was administered to clinically referred anxious children $(n=138)$ and control children $(n=38)$ as well as their parents.

Results: Results showed that the SCARED-71, child and parent report, differentiated clinically anxious from control children on total score and on all subscales. Reliable cut-off scores were established for both child and parent report. The parent version however had highest sensitivity and specificity and therefore seems the most reliable way to screen referred anxious children. Girls reported more anxiety symptoms than boys. Children aged 15-18 reported more anxiety symptoms compared to 8-14 year-olds. At subscale level, more symptoms of panic, generalised anxiety and social phobia were reported by the 15-18 year-olds, while situational phobia symptoms were less reported. Parents reported a decrease on separation anxiety, animal and situational phobia symptoms as children were older. Furthermore, evidence for the discriminant validity across anxiety disorders was found.

Conclusion: The SCARED-71 can be used as a screening tool to detect clinically anxious children and to discriminate specific anxiety disorders.
\end{abstract}

Keywords; Anxiety disorders, Assessment, Children 


\section{Introduction}

Are self-report questionnaires actually able to reliably detect childhood anxiety disorders? The Spence Children's Anxiety Scale (SCAS; Spence, 1998), Revised Child Anxiety and Depression Scale (RCADS; Chorpita et al., 2000) and Screen for Child Anxiety and Related Emotional Disorders (SCARED; Birmaher et al., 1997) seem to have potential for detecting anxiety problems in youths. An advantage of the SCARED-R(evised) (Muris et al., 1999a) is that it taps into all DSM-IV childhood anxiety disorders. Still, interviews such as the Anxiety Disorder Interview Schedule (ADIS; Silverman \& Nelles, 1988), the Diagnostic Interview Schedule (DISC; NIMH, 1992) and the Child version of the Structural Clinical Interview of DSM (KSCID; Hien et al., 1998) are necessary to establish an anxiety disorder classification. However, interviews are time-consuming, costly, and require trained professionals. With reliable cut-off scores, questionnaires like the SCARED-R might be helpful to screen for anxiety disorders and to differentiate nonclinical from clinically anxious children.

Evidence for the discriminant validity of the 66-item SCARED-R was obtained by differentiating between children with anxiety disorders, disruptive behaviour disorders, and mood disorders (Muris et al., 2003; Muris \& Steerneman, 2001). Furthermore, the chance of having an anxiety disorders was 9 times higher for children scoring high on the SCARED as compared to children with low scores (Muris et al., 2001). Using the original 38-item SCARED, Birmaher and colleagues (1997) found that anxiety-disordered youth also displayed significantly higher anxiety scores than children with other psychiatric disorders.

In order to differentiate children with severe and subclinical anxiety problems, reliable cut-off scores have to be obtained. Cut-off scores are useful in prevention research to identify children at high risk for anxiety problems. Furthermore, in therapy outcome studies, cut-off scores can be employed to determine whether treatment was successful in such way that a child has reached a subclinical level of anxiety. Muris et al. (2000) determined a cut-off point of 33 for the SCARED-R by means of discriminant analysis between subclinical and low anxious youth. However, it remains unclear whether this cut-off score holds for clinically anxious youth.

Two demographic factors are important when investigating anxiety in children, namely gender and age. Several studies have shown that girls report higher levels of anxiety than boys (e.g., Castellanos \& Hunter, 1999; Craske, 1997). Girls might report more anxiety symptoms because of gender stereotyping. That is, it is culturally more accepted that girls display anxiety symptoms. In addition, parental rearing styles may contribute to the fact that girls are allowed to display more anxiety. Moreover, girls' coping behaviour is more characterised by rumination and worry, whereas in boys active, problemoriented coping is more often used which is usually associated with less anxiety (Craske, 1997). Girls reported higher levels of anxiety symptoms on the 
SCARED-R as compared to boys in both a nonclinical as a clinical sample, and a similar trend was found on the parent version (Muris et al., 2003; Muris et al., 1999a; Muris et al., 1999b).

It is generally assumed that anxiety decreases as children become older (Bernstein et al., 1996; Castellanos \& Hunter, 1999). Initially, as children reach a certain level of cognitive development, their ability to perceive situations as potentially dangerous increases. With increasing age, however, the child gets a better understanding of these situations and learns to control them, which reduces anxiety (Ollendick et al., 1991). Studies using the SCARED-R with nonclinical children generally reveal no substantial age effects (e.g., Muris et al., 1998). In clinically anxious youths, symptoms of panic disorder and generalised anxiety increased with age, whereas symptoms of specific phobias and separation anxiety decreased, as children were older (Muris et al., 2003).

Three studies have examined whether the SCARED is able to reliably detect specific anxiety disorders in youths. Muris and colleagues (2000) found that children with subclinical specific phobia, generalised anxiety and separation anxiety symptoms on the DISC had significantly higher scores on the corresponding SCARED-66 subscale than children without such symptoms. Due to a small sample size, only the aforementioned subscales could be analysed. In another study by Muris and colleagues (2001), generalised anxiety disorder and separation anxiety disorder as classified by means of the KSCID were best predicted by high scores on the corresponding SCARED-R subscales in a non-clinical sample. Sensitivity and specificity scores were found to be modest to satisfactory. The social phobia subscale, however, had little predictive value, and other anxiety disorders were not investigated. In a study with clinically anxious children (Muris et al., 2003), further support for the predictive value of the SCARED-R was obtained.

This is the first study to investigate the psychometric properties of the SCARED-71 in a clinical population using both child and parent report. The SCARED-R did not sufficiently cover social phobia, therefore this subscale was enlarged by adding 5 extra items. The first goal was to examine the internal consistency of the SCARED-71. Special attention will be devoted to the altered social phobia subscale to investigate whether its reliability has improved. Second, the discriminant validity was investigated by comparing scores of clinical and nonclinical children and cut off scores were determined. Third, since several studies have shown that age and gender influence anxiety, effects of these demographic variables were also explored. Finally, the discriminant validity for detecting specific anxiety disorders will be investigated.

\section{Method}

\section{Participants}

Participants were clinically anxious $(n=138)$ and control children $(n=38)$. The clinically anxious children were referred for treatment and participated in a 
multi-centre randomized clinical trial comparing family with individual CBT (Bodden et al., submitted). Children were included, if their age was 8 to 18 $(M=12.5, S D=2.7)$, suffered from a primary anxiety diagnosis except for obsessive-compulsive disorder (OCD) and posttraumatic stress disorder (PTSD), and had an IQ $\geq 80$. Children were excluded if they suffered from substance abuse, suicide attempts, psychoses, autism spectrum disorders, and untreated attention deficit hyperactivity disorder (ADHD). There were 83 (60\%) girls and $55(40 \%)$ boys. Almost half $(44 \%)$ of the children attended primary school, the remaining 77 (56\%) attended secondary education. Of the parents, 136 mothers and 124 fathers participated.

The control sample consisted of children from the general population who were recruited via advertisements and were comparable to the clinically anxious group based on age, $F(1,174)=0.0, p>.1$ and gender, $x^{2}=0.1, p>.1$. Non-clinical children ranged in age from 8-18 $(M=12.4, S D=2.6)$. There were 24 $(63 \%)$ girls and $14(37 \%)$ boys. Primary education was attended by $15(40 \%)$ children and $23(60 \%)$ attended secondary education. All mothers $(n=38)$ and fathers $(n=38)$ participated.

The ADIS child $(\mathrm{C})$ and parent $(\mathrm{P})$ version were conducted to obtain diagnostic status. Primary and comorbid compound diagnosis of the clinically anxious children are shown in Table 1 . In the control group, $5 \%(n=2)$ had an anxiety disorder and $3 \%(n=1)$ ADHD.

Table 1 Primary and co-morbid diagnoses of the clinically anxious children on the ADIS $(n=138)$

\begin{tabular}{|c|c|c|c|c|c|c|c|c|}
\hline & \multicolumn{4}{|c|}{ Primary diagnosis } & \multicolumn{4}{|c|}{ Comorbid diagnosis } \\
\hline & \multicolumn{2}{|c|}{ ADIS-C } & \multicolumn{2}{|c|}{ ADIS-P } & \multicolumn{2}{|c|}{ ADIS-C } & \multicolumn{2}{|c|}{ ADIS-P } \\
\hline & $\bar{N}$ & $\%$ & $\bar{N}$ & $\%$ & $\bar{N}$ & $\%$ & $\bar{N}$ & $\%$ \\
\hline \multicolumn{9}{|l|}{ Diagnosis } \\
\hline No anxiety disorder & 6 & $4 \%$ & 0 & $0 \%$ & 0 & $0 \%$ & 0 & $0 \%$ \\
\hline Social phobia & 37 & $27 \%$ & 43 & $31 \%$ & 36 & $26 \%$ & 41 & $30 \%$ \\
\hline Separation anxiety disorder & 26 & $19 \%$ & 32 & $23 \%$ & 20 & $14 \%$ & 19 & $14 \%$ \\
\hline Specific phobia & 34 & $25 \%$ & 27 & $20 \%$ & 38 & $28 \%$ & 53 & $38 \%$ \\
\hline Generalised anxiety disorder & 25 & $18 \%$ & 25 & $18 \%$ & 22 & $16 \%$ & 40 & $29 \%$ \\
\hline Panic disorder & 10 & $7 \%$ & 11 & $8 \%$ & 13 & $9 \%$ & 11 & $8 \%$ \\
\hline \multicolumn{5}{|l|}{ Obsessive-compulsive disorder } & 5 & $4 \%$ & 5 & $4 \%$ \\
\hline \multicolumn{5}{|l|}{ Posttraumatic stress disorder } & 4 & $3 \%$ & 6 & $4 \%$ \\
\hline \multicolumn{5}{|c|}{ Depressive and/or dysthymic disorder } & 15 & $11 \%$ & 26 & $19 \%$ \\
\hline \multicolumn{5}{|c|}{ Attention-deficit hyperactivity disorder } & 3 & $2 \%$ & 9 & $7 \%$ \\
\hline \multicolumn{5}{|l|}{ Conduct disorder } & 0 & $0 \%$ & 2 & $1 \%$ \\
\hline \multicolumn{5}{|c|}{ Pervasive developmental disorder } & 0 & $0 \%$ & 1 & $1 \%$ \\
\hline \multicolumn{5}{|l|}{ Oppositional Defiant Disorder } & 0 & $0 \%$ & 2 & $1 \%$ \\
\hline
\end{tabular}




\section{Assessments}

\section{SCARED-71}

In this study, the SCARED-71 was used. The original 38-item SCARED measures separation anxiety, generalised anxiety disorder, panic disorder, social phobia and school phobia symptoms (Birmaher et al., 1997). Muris and colleagues (1999a) added items for measuring symptoms of specific phobia, OCD and PTSD. These alterations resulted in a 66-item questionnaire, the SCARED-R, which taps symptoms of all anxiety disorders as listed in the DSMIV (APA, 1994).

The SCARED-R is a reliable and valid questionnaire. The internal consistency of the total score is good in a nonclinical population (ranges from $\mathrm{a}=.91$ to $\mathrm{a}=.95$ ) but slightly lower in a clinical sample (Muris, et al., 1999b, Muris et al., 2003, Muris \& Steerneman, 2001). The test-retest validity is satisfactory (Muris, et al, 1999b) and the concurrent validity is good (Muris et al., 1998). Finally, the treatment sensitivity of the SCARED-R has proven to be adequate (Muris et al., 1999c).

Despite the good psychometric properties of the SCARED-66, the social phobia subscale of the SCARED-R only consists of four items, which can be regarded as rather insufficient compared to the other subscales. Further, these four items all refer to fear of interaction with unfamiliar people. However, socially phobic children often also fear encounters with familiar people and social performance situations (Bögels \& van Melick, 2004). To overcome this shortcoming, 5 social phobia items were added, based on items of the SCAS and the Social Phobia and Anxiety Inventory for Children (SPAIC; Beidel et al., 1995). On the SCARED-71, children and parents have to indicate how frequently the child experiences each anxiety symptom using a 3-point Likert scale indicating almost never (0), sometimes (1) and often (2). ADIS

In both samples, a semi-structured diagnostic interview was conducted, namely the ADIS. This interview is specifically designed to measure anxiety disorders in 7-17 year-olds. For each diagnosis a severity score can be obtained that ranges between 0 and 8 , with 4 or higher indicating a clinical diagnosis. The ADIS-C/P possesses good inter-rater (Silverman \& Nelles, 1998) and high testretest reliability (Silverman \& Eisen, 1992). The research assistants who performed the ADIS interviews in our study videotaped 4 of their interviews, which were then re-rated by 2 trained psychology students. The mean interrater agreement (kappa) was 0.89 for the ADIS-C and 0.83 for the ADIS-P.

\section{Procedure}

All children and parents signed an informed consent. Clinically anxious children were assessed before and after treatment and at follow-up. Families from the general population were measured once and received a financial reward afterwards. All family members filled out a battery of questionnaires, 
including the SCARED-71. Children were assisted if necessary by a research assistant.

\section{Analysis}

Missing data were extrapolated using the missing value analysis of SPSS based on regression models using available data of the group mean, the available data of the individual and the pattern of change of the group. The SCARED-71 scores of father and mother were averaged. Group differences on total SCARED scores were analyzed by means of the Analyses of (Co)Variance (AN(C)OVA). To evaluate differences on the subscales, a Multivariate ANCOVA (MANCOVA) was carried out. A discriminant analysis was performed to predict the diagnoses of the specific anxiety disorders. In addition, cut-off scores were established using ROC curve analysis.

\section{Results}

\section{Internal consistency}

Firstly, internal consistency coefficients were computed separately for the child, mother and father version using the total sample. Cronbach's alpha coefficients were high, indicating a high degree of homogeneity. The internal consistencies of the subscales were moderate to high, with Cronbach's alpha values ranging from .64 (situational-environmental phobia; child version) to .93 (animal phobia; father version). The internal consistency coefficients of the extended social phobia subscale were all high: .85 for child, .91 for mother and .89 for father report (see Table 2).

Table 2 Cronbach's alpha values on the SCARED-71 total and subscales scores for the child $(n=$ 176), mother $(n=174)$ and father version $(n=155)$

\begin{tabular}{llll}
\hline & Child & Mother & Father \\
\hline Subscale & & & \\
Panic disorder & .88 & .88 & .86 \\
Generalised anxiety Disorder & .87 & .87 & .87 \\
Social phobia & .85 & .91 & .89 \\
Separation anxiety disorder & .81 & .86 & .84 \\
Obsessive-compulsive disorder & .74 & .77 & .70 \\
Posttraumatic stress Disorder & .82 & .84 & .80 \\
Animal phobia & .87 & .92 & .93 \\
Blood-injection-Injury phobia & .74 & .80 & .83 \\
Situational-environmental phobia & .64 & .66 & .67 \\
Total score & .95 & .96 & .95 \\
\hline
\end{tabular}




\section{Discriminant Validity}

The discriminant validity of the SCARED-71 was examined by comparing total scores of clinically anxious youths with those of control youths by means of an ANCOVA with gender and age as covariates. Results indicated that clinically anxious children reported significantly more anxiety symptoms than control children, $F(1,172)=58.8, p<.01$, pooled effectsize $=1.6$. A MANCOVA with the subscales as dependent variables revealed a main effect for group as well, $F_{\text {Hotelling }}(9,164)=6.9, p<.01$. Follow-up ANOVAs showed that anxious children exhibited significantly higher levels of anxiety symptoms on all subscales as compared to control children, all $F(1,172)>9.8, p<.01$ (see Table 3).

In line with these results, the parents of clinically anxious children reported higher levels of anxiety symptoms in their children than the parents of control youth, $F(1,172)=123.8, p<.01$, pooled effectsize $=2.4$. Again, this pattern was found across individual subscales, $F_{\text {Hotelling }}(9,164)=17.5, p<.01$, indicating that according to their parents, clinically anxious children exhibited higher levels of all anxiety symptoms as compared to control children, as all $F(1,172)>11.4, p<.01$ (see Table 3 ).

Table 3 Mean SCARED-71 scores and standard deviations in the clinically anxious sample (children $n=138$, mothers $n=136$ and fathers $n=124$ ) and the control sample (children $n=38$, mothers $n=38$ and fathers $n=38$ )

\begin{tabular}{|c|c|c|c|c|c|}
\hline & \multicolumn{2}{|c|}{ Clinically anxious } & \multicolumn{2}{|c|}{ Control } & \multirow[b]{2}{*}{$F$} \\
\hline & $M$ & $S D$ & $M$ & $S D$ & \\
\hline \multicolumn{6}{|l|}{ Child version } \\
\hline Panic disorder & 7.4 & 5.7 & 2.1 & 2.7 & 31.1 \\
\hline Generalised anxiety disorder & 7.8 & 4.5 & 3.7 & 3.2 & 26.9 \\
\hline Social phobia & 8.2 & 4.5 & 4.3 & 3.2 & 24.6 \\
\hline Separation anxiety disorder & 8.2 & 5.1 & 3.2 & 2.4 & 34.0 \\
\hline Obsessive compulsive disorder & 6.2 & 3.6 & 2.9 & 2.0 & 29.4 \\
\hline Posttraumatic stress disorder & 2.3 & 2.4 & 1.0 & 1.3 & 9.7 \\
\hline Animal phobia & 1.6 & 2.0 & 0.3 & 0.7 & 14.2 \\
\hline Blood-injection-Injury phobia & 4.4 & 3.3 & 2.6 & 2.3 & 10.2 \\
\hline Situational-environmental phobia & 3.5 & 2.5 & 1.6 & 1.6 & 20.9 \\
\hline Total score & 49.6 & 22.3 & 21.7 & 12.2 & 54.7 \\
\hline \multicolumn{6}{|l|}{ Parent version Combined } \\
\hline Panic disorder & 6.5 & 4.8 & 0.6 & 0.9 & 55.9 \\
\hline Generalised anxiety disorder & 8.6 & 3.7 & 2.6 & 2.0 & 92.8 \\
\hline Social phobia & 8.6 & 4.4 & 2.8 & 2.3 & 62.5 \\
\hline Separation anxiety disorder & 9.4 & 4.8 & 1.8 & 1.5 & 93.5 \\
\hline Obsessive compulsive disorder & 4.8 & 2.9 & 1.0 & 0.9 & 62.5 \\
\hline Posttraumatic stress disorder & 2.4 & 2.1 & 0.5 & 0.7 & 31.1 \\
\hline Animal phobia & 1.3 & 1.8 & 0.3 & 0.8 & 11.1 \\
\hline Blood-injection-Injury phobia & 4.2 & 3.3 & 1.9 & 2.4 & 16.6 \\
\hline Situational-environmental phobia & 3.2 & 2.2 & 1.0 & 1.1 & 32.6 \\
\hline Total score & 49.0 & 19.7 & 12.6 & 7.7 & 123.8 \\
\hline
\end{tabular}

Note: all $p \mathrm{~s}<0.01$ 


\section{Gender}

To test gender effects on the total SCARED-71, an ANCOVA was carried out with group and age as covariates. Girls reported more anxiety symptoms $(M=$ $46.3, S D=24.4)$ compared to boys $(M=39.2, S D=21.5), F(1,172)=4.7, p<.05$, pooled effectsize $=0.3$. No interaction effects with group, $F(1,168)=0.6$ or age, $F(1,168)=0.0$ were found. Across subscales, no significant differences between boys and girls were found, $F_{\text {Hotelling }}(9,164)=1.6, p>$. 1. Furthermore, no significant group by gender and age by gender interaction effects were revealed, respectively $F_{\text {Hotelling }}(9,160)=0.6, p>.1$ and $F_{\text {Hotelling }}(9,160)=0.7, p>.1$. Parents reported no differences between boys and girls on either the total score, $F(1,172)=1.0, p>.1$ or the subscales, $F_{\text {Hotelling }}(9,164)=1.3, p>.1$.

Age

In order to examine age effects corrected for gender and group, an ANCOVA was carried out in which age was categorised into 3 groups, namely 8-11, 12-14, and 15-18 year-olds. A significant age effect was found, $F(1,172)=3.8, p=.05$. Further comparisons between the three age groups showed that in particular $15-18$ year-olds differed from 8-11 year-olds, $F(1,113)=5.7, p<.05$ and $12-14$ year-olds, $F(1,103)=11.8, p<.01$. The $8-11$ year-olds and $12-14$ year-olds did not differ significantly, $F(1,130)=1.4, p>.05$. No interaction effect with group, $F(1,168)=0.0$ was found.

A MANCOVA performed on the subscales also showed a significant age effect, $F_{\text {Hotelling }}(9,164)=11.6, p<.01$. Follow-up ANOVAs showed age differences on the subscales panic, $F(1,172)=22.4, p<.01$, generalised anxiety, $F(1,172)=26.7, p<.01$, social phobia, $F(1,172)=21.1, p<.01$ and situational phobia, $F(1,172)=11.9, p<.01$. For all anxiety disorders except for situational phobia, a similar pattern was found: that is 8-11 and 12-14 year-olds did not differ significantly, all $F(1,130)<1.5, p>.05)$, whereas the highest scores were found for 15-18-year-olds compared to 8-11 year-olds, all $F(1,113)>21.7, p<.01$, and compared to 12-14 year-olds, all $F(1,103)>12.4, p<.01$. Scores on situational phobia appeared to decrease with age and differed significantly between $8-11$ and 15-18 years-old, $F(1,113)>9.1, p<.01$. No interaction effect of age by group was found, $F_{\text {Hotelling }}(9,160)=0.3$.

No age differences were found for the total parent scores, $F(1,172)=1.3$, $p>$.1. Also, no interaction effect with group was found, $F(1,168)=0.0$. However, for the subscales, differences between age groups did emerge, $F_{\text {Hotelling }}(9,164)=6.1, p<.01$. Follow-up ANOVAs showed that with increasing age, parents reported lower scores on their children's symptoms of separation anxiety, $F(1,172)=7.2, p<.01$, animal phobia, $F(1,172)=4.9, p<.05$, and situational phobia, $F(1,172)=27.6, p<.01$. Again, no interaction effect with group was found, $F_{\text {Hotelling }}(9,160)=0.2$. 


\section{Cut-off scores}

In order to differentiate between clinically anxious and control children by means of the SCARED-71 total score, cut-off scores were established. This cutoff score was obtained by determining the maximum sensitivity and specificity score of the SCARED-71 for predicting any ADIS anxiety diagnosis by means of ROC curve analysis. The optimal cut-off score for the total SCARED-71 child version was 30 . The sensitivity was 0.78 , which indicates that $78 \%$ of the children who score above the cut-off part on the scale were classified as having an anxiety disorder, and really suffered from significant anxiety problems. There were $22 \%$ false negatives: these children were not detected by the SCARED-71 as potentially having an anxiety disorder but actually displayed serious anxiety complaints. The specificity of the SCARED-71 child version was 0.76 , which means that $76 \%$ of the children who scored below the cut-off part were not classified as having an anxiety disorder and indeed did not have anxiety problems. There were $24 \%$ false positives, which refer to youths classified by the SCARED-71 child version as possibly having an anxiety disorder, but actually do not display serious anxiety complaints. For the parent version, the optimal cut-off score was 21 . Sensitivity and specificity were both 0.92 . The parent and child version combined revealed a cut-off score of 27 , with sensitivity of 0.89 and specificity of 0.90 . In Table 4 , cut off scores for various gender and informant groups are shown.

Table 4 Total SCARED-71 cut-off scores based on the clinically anxious sample (children $n=138$, mothers $n=136$ and fathers $n=124$ ) and the control sample (children $n=38$, mothers $n=38$ and fathers $n=38$ )

\begin{tabular}{lccc}
\hline & Cut-off score & Sensitivity & Specificity \\
\hline Child version & 30 & 0.78 & 0.76 \\
Child version-boys & 27 & 0.71 & 0.69 \\
Child version-girls & 31 & 0.82 & 0.80 \\
Parent version combined & 21 & 0.92 & 0.92 \\
Parent version-mother & 25 & 0.85 & 0.87 \\
Parent version-father & 22 & 0.88 & 0.87 \\
Combined child and parent & 27 & 0.89 & 0.90 \\
\hline
\end{tabular}

\section{Discriminant analysis}

In order to investigate whether specific anxiety diagnoses were best predicted by their corresponding SCARED-71 subscale, a discriminant classification analysis was carried out only using data of clinically referred youth. Children with a primary diagnosis of OCD and PTSD were not included, therefore these disorders were not taken into account. The results demonstrated that panic disorder, social phobia, separation anxiety disorder and specific phobia as measured with the ADIS-C were best predicted by their corresponding subscale of the SCARED-71 child version, all $p$ s $<.05$. The corresponding subscale of the 
SCARED-71 had the highest loading factor on the standardised canonical discriminant function. The subscale generalised anxiety was found to be a borderline significant predictor for the resembling ADIS-C diagnosis. Sensitivity and specificity scores were moderate to high for all subscales, indicating satisfactory to good classification.

Anxiety disorders as obtained with the ADIS-P were best predicted by their corresponding subscale on the SCARED-71 parent version, all $p s<.05$. The highest standardised canonical discriminant function coefficient of each subscale resembled the concerned anxiety diagnosis. The sensitivity and specificity scores of the subscales were moderate to high (see Table 5).

Table 5 Discriminant classification of the SCARED-71 in clinically anxious children self-report $(n=$ 138) and parent-report $(n=138)$

\begin{tabular}{|c|c|c|c|c|c|c|}
\hline & $\begin{array}{l}+ \text { Dis } \\
M(S D)\end{array}$ & $\begin{array}{l}\text { - Dis } \\
M(S D)\end{array}$ & $\begin{array}{l}\text { Wilks' } \\
\text { Lambda }\end{array}$ & SCDF & Sens & Spec \\
\hline \multicolumn{7}{|l|}{ Child version } \\
\hline Panic & $11.8(7.0)$ & $6.5(5.0)$ & $.84^{*}$ & 1.04 & 0.70 & 0.79 \\
\hline Generalised anxiety & $9.3(4.6)$ & $7.0(4.3)$ & $.88^{* *}$ & 0.87 & 0.64 & 0.69 \\
\hline Social phobia & $10.0(4.2)$ & $7.1(4.4)$ & $.70^{*}$ & 1.12 & 0.75 & 0.75 \\
\hline Separation anxiety & $12.3(4.8)$ & $6.1(3.9)$ & $.57^{*}$ & 0.78 & 0.80 & 0.85 \\
\hline \multicolumn{7}{|c|}{ Specific Phobia } \\
\hline Animal & $2.0(2.2)$ & $1.2(1.8)$ & $.83^{*}$ & 0.33 & 0.67 & 0.71 \\
\hline Medical & $5.4(3.4)$ & $3.4(2.9)$ & $.83^{*}$ & 0.40 & 0.67 & 0.71 \\
\hline Situational & $4.3(2.6)$ & $2.7(2.2)$ & $.83^{*}$ & 0.43 & 0.67 & 0.71 \\
\hline \multicolumn{7}{|l|}{ Parent version } \\
\hline Panic & $10.5(5.5)$ & $5.7(4.3)$ & $.74^{*}$ & 0.98 & 0.68 & 0.78 \\
\hline Generalised anxiety & 10.5 (3.2) & $6.9(3.2)$ & $.70^{*}$ & 1.04 & 0.78 & 0.81 \\
\hline Social phobia & $10.7(3.6)$ & $5.5(3.6)$ & $.60^{*}$ & 0.86 & 0.81 & 0.81 \\
\hline Separation anxiety & $12.8(4.5)$ & $7.4(3.7)$ & $.58^{*}$ & 0.96 & 0.78 & 0.84 \\
\hline \multicolumn{7}{|l|}{ Specific Phobia } \\
\hline Animal & $1.7(2.2)$ & $1.3(1.8)$ & $.66^{*}$ & 0.15 & 0.74 & 0.83 \\
\hline Medical & $5.4(3.4)$ & $4.2(3.3)$ & $.66^{*}$ & 0.41 & 0.74 & 0.83 \\
\hline Situational & $4.1(2.6)$ & $3.2(2.2)$ & $.66^{*}$ & 0.70 & 0.74 & 0.83 \\
\hline
\end{tabular}

Note: + Dis $=$ with specific anxiety disorder; - Dis $=$ without specific anxiety disorder; SCDF $=$ Standardised Canonical Discriminant Function Coefficient; Sens. = Sensitivity, and Spec. = Specificity, ${ }^{*} p<0.05$

\section{Discussion}

The current study examined the diagnostic utility of the SCARED-71 in both clinically anxious and control youths and their parents. The main results can be summarised as follows. First, the SCARED-71 possessed good internal consistency. Second, in line with the hypothesis, the child and parent version of the SCARED-71 differentiated clinically anxious from normal children on the total score and on all subscales. Third, girls reported more anxiety symptoms 
than boys, but only on self-report. Fourth, children aged 15-18 reported a higher degree of anxious symptoms as compared to 8-14 year-olds. At subscale level, children reported more panic, generalised anxiety disorder and social phobia symptoms with older age groups, while situational phobia symptoms were lower in the older age groups. Parents reported more anxiety symptoms relating to separation anxiety, animal phobia and situational phobia, as children were older. Fifth, reliable cut-off scores were obtained. Finally, the SCARED-71 discriminated across various anxiety disorders.

The internal consistencies of the SCARED-71 child and parent version were, in line with research of Muris and colleagues (e.g. Muris et al, 2003), high except for situational-environmental phobia. The latter subscale consists of a wide variety of stimuli like height, thunder and darkness, which is likely to decrease homogeneity. The Cronbach's alpha scores on the subscale social phobia were slightly higher, compared to SCARED-R studies (Muris et al, 1999b; Muris et al., 2003), therefore the altered social phobia subscale seems more reliable.

The child and parent version of the SCARED-71 differentiated clinically anxious from non-clinical children on the total score and on all subscales. This finding is in line with earlier studies using different SCARED versions (Muris et al., 2003; Muris et al., 2001). More over, previous research has shown that the DSM-based scale can differentiate between children with anxiety disorders and children with psychiatric disorders (Birmaher et al., 1997), disruptive disorders (Muris \& Steerneman, 2001) and mood disorders (Muris et al., 2003). Hence, the majority of the data support the notion that the SCARED and its revisions have good discriminant validity.

In both clinical and control sample, girls reported higher levels of anxiety symptoms on the total SCARED-71 than boys. This finding is in line with previous studies showing that girls report more anxiety than boys (e.g., Castellanos \& Hunter, 1999; Craske, 1997). Girls might report more anxiety symptoms because of gender stereotyping, cultural acceptance, parenting styles which promote anxiety in girls more and the more internalising coping style of girls which results in more anxiety (Craske, 1997). A study of Pierce and Kirkpatrick (1992) showed that when male students are given instructions that their truthfulness will be measured by means of changes in heart rate, their fears on a fear schedule increased while no such increase was found in female students. This implies that males as do boys may fill in fear and anxiety questionnaires in a cultural desirable way.

In line with other studies using different questionnaires, no gender differences were observed when using the parent version of the SCARED-71 in both the clinical and the control group (Straus \& Last, 1993; Last et al., 1992). The lack of gender difference in parent report in the clinical group can be explained by the fact that parents perceive the anxiety symptoms of girls as normal and therefore are not inclined to search treatment unless the anxiety is very severe. If boys display anxiety symptoms parents might be more prone to 
seek help. Therefore, the difference in gender might be less pronounced than in a normal sample (Craske, 1997).

With respect to age, the finding that self-reported anxiety is higher in 15-18 year-old clinically anxious children compared to 8-11 and 12-14 year-olds, is remarkable. From a clinical practice point of view, this finding can be explained by the fact that under the age of 12, parents are usually the ones who bring their child to a mental health care facility. The impact of the child's anxiety will be better noticed by the parents than by the child itself. When the child researches puberty, he or she becomes more autonomous and will react against its parents and institutions like mental health care centres. Also the child might be afraid of reactions of peers and of stigmatising, which increases the threshold value for an older child to go to a mental health care centre. Therefore, only the teenagers with self-experienced severe anxiety symptoms may agree with referral for mental health care. The same age pattern was found for the control children, 15 to 18-year olds reported the highest anxiety symptoms. This could be explained by the fact that these adolescents are in a period of their lives in which they are approaching important life changes like independent living and work, which is found to increase anxiety (e.g. Caspi, Elder, \& Bem, 1988). Perhaps, adolescents aged 15 to 18 might also fill in questionnaires less social desirable. A larger sample and a longitudinal design are necessary to further investigate this age effect.

At subscale level, more symptoms of panic, generalised anxiety and social phobia symptoms were reported by the 15-18 year old children, while situational phobia symptoms were less reported. Parents reported more anxiety symptoms relating to separation anxiety, animal phobia and situational phobia, in the 8-11 year olds. This is in line with the reported age of onset of anxiety disorders with the earliest mean age of onset for separation anxiety and specific phobia, while the age of onset for social phobia is approximately 10 years and the onset of generalised anxiety disorder is found to range between 10.8 to 13.4 years. Panic disorder and agoraphobia are often only found in adolescence (Castellanos \& Hunter, 1999; Craske, 1997). In line, a study examining physical and social evaluative fears separately showed that physical fears decreased with age, whereas social-evaluative fears increased with age (Westenberg et al., 2004).

The difference in age effect between child and parent report for different anxiety disorders can be explained by the fact that parents can observe anxious behaviour related to separation anxiety and phobia more clearly than that of more internalising anxiety disorders as generalised anxiety disorder. Besides this, separation anxiety is also likely to intervene with parents' functioning, while social phobia and generalised anxiety mostly hampers the child's functioning.

The cut-off scores of the SCARED-71, obtained in this study, can be useful for research and clinical practice to detect clinically anxious children and to evaluate high end state functioning in treatment studies. Sensitivity and 
specificity scores were reasonably high, reflecting a reliable identification of children possibly having an anxiety disorder. The highest sensitivity and specificity scores in our study were found for the parent version. With a cut-off score of 21 , the parent version seems to be the most reliable way to screen clinically referred anxious children, because the percentage of false positives and false negatives is the smallest compared to the child version or the combined version. This finding might be in line with aforementioned point that parents are usually the ones who bring the child to mental health care while the child might not want treatment or think its' anxiety problems are not severe enough.

In the clinical sample, anxiety disorders were best predicted by the corresponding SCARED-71 subscale. This indicates that in general, subscales scores are a reliable indicator of the persistent clinical diagnoses. Only generalised anxiety symptoms reported by children were a borderline significant predictor of actual diagnosis. This can be explained by the fact that symptoms of generalised anxiety are less specific and show the same features as other anxiety disorders symptoms, which causes overlap with other anxiety disorders.

This study had some limitations. First, children with OCD and PTSD as a primary disorder were not included, therefore discriminant classification could not be obtained for these disorders. Second, age effects were measured cross sectional, while a longitudinal study is preferred to measure such effects. Third, most children were Caucasian, therefore ethnicity could not be taken into account and results can only be generalised to a Caucasian population. Fourth, although this study shows that the SCARED-71 is a good instrument to screen for anxiety disorders in children, no conclusions can be drawn related to other anxiety questionnaires. Hence, future research should compare the SCARED-71 to other anxiety questionnaires like the SCAS and RCADS to investigate which questionnaire is most reliable and valid to detect children with clinically anxious problems.

This study has shown that the SCARED-71 can be used as a screening tool in research and clinical practice. The SCARED-71 total score cannot only discriminate between control and clinically anxious children, there is also evidence for the discriminate validity of the SCARED-71 subscales across anxiety disorders. 


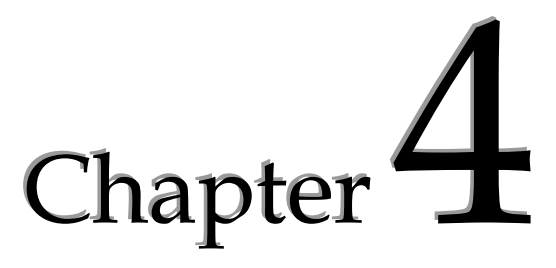

Negative automatic thinking in clinically anxious and control youth: The effects of gender, age and contentspecificity

Denise H.M. Bodden \& Susan M. Bögels 


\begin{abstract}
This study examined the frequency of negative automatic thoughts concerning physical threat, social threat, personal failure and hostile intent in clinically anxious $(n=139)$ and control children $(n=61)$ by means of the Children's Automatic Thoughts Scale (CATS; Schniering \& Rapee, 2002). In line with the hypothesis, it was found that clinically anxious children reported more negative automatic thoughts compared to control children. On subscale level, clinically anxious children reported more negative automatic thoughts relating to physical threat, social threat and personal failure than control children, whereas no difference on the report of negative thoughts concerning hostile intent was found. Girls reported more negative thoughts on the subscale social threat, whereas boys reported more thoughts on hostile intent. In 15-18 year-olds, the highest frequency of negative automatic thoughts was found, compared to 8-11 and 12-14 year-olds. The two latter did not differ significantly on frequency of negative thoughts. Some evidence for content specificity of automatic thought patterns was found, that is separation anxiety was best predicted by the physical threat subscale, social phobia was best predicted by social threat and depression was best predicted by the personal failure subscale of the CATS.
\end{abstract}

Keywords; Anxiety disorders, Children, Cognitive assessment 


\section{Introduction}

The vast majority of effectiveness studies on Cognitive Behavioural Therapy (CBT) in children with anxiety disorders use anxiety questionnaires or interviews to measure change in anxiety symptoms, but do not measure possible changes in anxious children's cognitions. As a result, it is unknown whether the demonstrated effects on anxiety are related to change in cognitions. To investigate whether cognition is an important mediator for change, reliable and valid assessment instruments are essential.

There are several self-report measures designed to assess dysfunctional thinking in children with anxiety and depression problems, e.g. the Negative Affect Self Statement Questionnaire (NASSQ; Ronan, Kendall \& Rowe, 1994) and the Children's Thoughts Questionnaire (CTQ; Marien \& Bell, 2004). However, these questionnaires do not measure thoughts across internalizing and externalizing problems. Schniering and Rapee (2002) have developed the Children's Automatic Thought Scale (CATS), which is a developmentally sensitive self-report measure designed to assess a wide range of negative automatic thoughts across internalizing and externalizing problems in children and adolescents, aged 7-16. The CATS consists of four distinct but related subscales namely physical threat, social threat, personal failure and hostile intent, which load onto one higher order factor.

Some studies have addressed the issue if anxious children can be characterised by having more negative thoughts compared to children from the general population. Most of them found that high anxious children report more negative cognitions than low anxious children (e.g. Ronan, Rowe \& Kendall, 1988; Zatz \& Chassin, 1983). In line, results of Schniering and Rapee (2002) showed that anxious, depressed and behavioural disordered children reported significantly more negative automatic thoughts on the CATS than children from a community sample. On the CATS subscale level, anxious children had higher scores for physical threat, social threat and personal failure compared to the community children, whereas no difference was found on hostile intent. In another line of cognition research namely free recall, anxious children expressed more negative than neutral and positive thoughts related to an ambiguous situation, whereas control children expressed equal numbers of negative, neutral and positive thoughts (Bögels \& Zigterman, 2000). Kendall (1984) has called this phenomenon, the power of nonnegative thinking, which implies that a lower frequency of negative cognitions instead of the presence of positive cognitions differentiates normal children from maladaptive children. Hence the amount of negative cognitions seems to be larger in children with anxiety disorders than in children without anxiety disorders.

Two factors are important when investigating cognition in children, namely gender and age. In general, anxiety and mood disorders are more frequent in girls, whereas disruptive behaviours are more frequent in boys (Verhulst, Van der Ende, Ferdinand, \& Kasius, 1997). However, Schniering and 
Rapee $\left(2002 ; 2004^{\mathrm{a}} ; 2004^{\mathrm{b}}\right.$ ) did not find a gender effect on the CATS total score in a community sample. Although, on subscale level, boys reported more hostile intent thoughts, while girls reported significantly more automatic thoughts on personal failure and social threat (Schniering \& Rapee, 2002). The latter finding is in line with Campbell and Rapee (2004) who found that social threat cognitions were more frequent in girls.

It remains unclear whether negative thinking is related to age. Weems, Berman, Silverman, and Saavedra (2001) found a stronger association of cognitive distortions and anxiety in 12-17 year-olds compared to 6-11 year-olds. Also, results of several studies show that older children tend to have more catastrophising and personal cognitions (Prins, 2002). In contrast, no age difference was revealed on the CATS in a community sample (Schniering \& Rapee, 2002; 2004a; 2004b). Similar results were shown in a study on loss and threat cognitions (Ambrose \& Rholes, 1993). These inconsistent results warrant further investigation of the effect of age on cognitions in anxious children.

The content of the CATS subscales is based on Beck's cognitive specificity model of anxiety. This model implies that thoughts are organised into distinct cognitive contents. These contents are specifically related to the expression of certain psychopathology. Thoughts on failure, loss and deprivation are associated with depression, whereas threat, danger and unpredictability are associated with anxiety and thoughts of being wronged are associated with anger (Beck, Brown, Steer, Eidelson, \& Riskind, 1987; Beck \& Clark, 1988).

Schniering and Rapee (2002; 2004a) found some evidence for the cognitive content-specificity of the CATS. Anxiety disorders were associated with social threat, depression associated with personal failure and behavioural disorders with hostile intent. However, in one study, physical threat was associated with anxiety disorders (Schniering \& Rapee, 2002), but not in another study (Schniering \& Rapee, 2004a). Furthermore, depressed children reported more automatic thoughts on personal failure than did anxious, normal and disruptive children (Schniering \& Rapee, 2004a). The finding that anxiety is related to threat cognitions and depression is associated with personal failure cognitions is in line with other studies (Ambrose \& Rholes, 1993; Jolly 1993; Jolly \& Dykman, 1994; Marien \& Bell, 2004). However, some studies did not find evidence for the cognitive specificity hypothesis (Epkins, 1996; Ronan \& Kendall, 1997).

Also within anxiety disorders there is some evidence for content specificity. A study by Bögels, Snieder and Kindt (2003) showed that separation anxious children interpreted ambiguous stories in line with their fear compared to social phobic children and generalised anxious children. Social phobic children overestimated the danger of rejection compared to separation anxious children but not when compared to generalised anxious children. No support for the content-specificity hypothesis was found for generalised anxiety. Results of a study on social phobia showed that children with social phobia had a 
higher level of negative self-talk regarding social evaluative situations compared to non-anxious children (Spence, Donovan \& Brechman-Toussaint, 1999). Further research seems necessary to further explore the content specificity hypothesis within anxiety disorders in order to enhance effectiveness of cognitive behaviour therapy by targeting specific thoughts.

The main purpose of this study was to investigate the utility of the CATS to measure negative automatic thoughts in a clinically anxious and control sample. Firstly, consistent with the results of Schniering and Rapee (2002) it was predicted that clinically anxious children have more negative automatic thoughts on the total score, and on the subscales physical threat, social threat, and personal failure in comparison to the control sample. Secondly, it was hypothesised that girls have more negative thoughts on social threat and personal failure, while boys report more thoughts on hostile intent. Thirdly, gender and age differences are investigated. Fourthly, we investigated whether children with different types of anxiety disorders can be characterised by different types of automatic thoughts. As separation anxiety is being described as "worry that something might happen to them or to a significant other" (DSM-IV, APA, 1994), we hypothesised that separation anxiety in children would be predicted by the physical threat subscale. Social phobia is predicted to be related to the social threat subscale of the CATS. It is further hypothesised that panic disorder and specific phobia are best predicted by high scores on physical threat. Generalised anxiety disorder is predicted to be related to physical threat, social threat and personal failure, since these areas are all associated with worry. Fifthly, according to the cognitive specificity model, it was predicted that anxious children with comorbid depression would have higher scores on personal failure than anxious children without comorbid depression.

\section{Method}

\section{Participants}

Participants were clinically anxious children $(n=139)$ and control $(n=61)$ children. The clinical sample consisted of referred children and adolescents who took part of a multi-centre randomized clinical trial comparing family CBT with individual CBT (Bodden et al., submitted). Children were included if they were 8-18 years old, suffered from a primary anxiety diagnosis (other than obsessive compulsive disorder and post-traumatic stress disorder), and had an intelligence coefficient of $\geq 80$. Children were excluded if they suffered from substance abuse, current suicide attempts, psychoses, autism spectrum disorders and untreated attention deficit hyperactivity disorder. There were 82 $(59 \%)$ girls and $57(41 \%)$ boys, with a mean age of $12.5(S D=2.7)$. Sixty children $(43 \%)$ attended primary education. The remaining $79(57 \%)$ attended secondary education. 
The control sample consisted of children from the general population who were recruited via advertisements in newspapers and local stores and received a financial reward of $€ 50$ for their participation. Control children were comparable to the clinically anxious group based on age, $F(1,198)=.67, p>.1$ and gender, $x^{2}=2.9, p>.1$. The age of the control children ranged from 8-18 years $(M=12.2, S D=2.6)$. There were $28(46 \%)$ girls and $33(54 \%)$ boys. Primary education was attended by $24(39 \%)$ children and $37(61 \%)$ received secondary education.

To obtain diagnostic status, both child and parent version of the Anxiety Disorder Interview Schedule (ADIS c/p; Silverman \& Nelles, 1988) were assessed. Primary and comorbid compound diagnosis of the clinical anxious children are outlined in Table 1 . In control children, the prevalence of anxiety disorders was 3\% (2 out of 61) and 2\% (1 out of 61) for Attention Deficit Hyperactivity Disorder.

Table 1 Primary and comorbid diagnosis of the clinical sample on the compound ADIS $(n=139)$

\begin{tabular}{|c|c|c|c|c|}
\hline & \multicolumn{2}{|c|}{ Primary diagnosis } & \multicolumn{2}{|c|}{ Comorbid diagnosis } \\
\hline & $N$ & $\%$ & $N$ & $\%$ \\
\hline \multicolumn{5}{|l|}{ Diagnosis } \\
\hline Social phobia & 46 & $33 \%$ & 48 & $35 \%$ \\
\hline Separation anxiety disorder & 34 & $25 \%$ & 24 & $17 \%$ \\
\hline Specific phobia & 24 & $17 \%$ & 58 & $42 \%$ \\
\hline Generalised anxiety disorder & 24 & $17 \%$ & 46 & $33 \%$ \\
\hline Panic disorder & 11 & $8 \%$ & 24 & $17 \%$ \\
\hline Obsessive-compulsive disorder & & & 6 & $4 \%$ \\
\hline Posttraumatic stress disorder & & & 6 & $4 \%$ \\
\hline Depressive and/or dysthymic disorder & & & 26 & $19 \%$ \\
\hline Attention Deficit Hyperactivity disorde & & & 11 & $8 \%$ \\
\hline Conduct Disorder & & & 1 & $1 \%$ \\
\hline Oppositional Defiant Disorder & & & 1 & $1 \%$ \\
\hline Pervasive Developmental Disorder & & & 1 & $1 \%$ \\
\hline
\end{tabular}

Measures

\section{CATS}

All participants completed a translation of the Children's Automatic Thought Scale (CATS; Schniering \& Rapee, 2002). Children and adolescents have to indicate on a 5-point scale, the frequency with which they have experienced each thought over the past week. The scale ranges from not at all (0) to all the time (4). The 40 items are summed to provide a total score, with high scores reflecting a greater frequency of negative automatic thoughts. The CATS consists of 4 subscales: physical threat (e.g. "I'm going to get hurt"), social threat (e.g. "I look like an idiot"), personal failure (e.g. "I am worthless") and hostile intent (e.g. "Most people are against me"). The CATS has a good internal consistency and test-retest reliability (Schniering \& Rapee, 2002, 2004). 
ADIS

In both samples, a semi structured diagnostic interview was administered, namely the ADIS child and parent version. This interview is specifically designed to measure anxiety disorders, depression and externalising disorders in 7-17 year-olds. Each diagnosis has a severity score that ranges between 0 and 8 , with a score of 4 or higher indicating a clinical diagnosis. The child and parent version were combined into a compound summary. The ADIS-C/P possesses good inter-rater (Silverman \& Nelles, 1998) and high test-retest reliability (Silverman \& Eisen, 1992). Research assistants in our study had to videotape 4 own interviews, which were re-rated by 2 trained students. The mean inter-rater agreement for diagnoses (kappa) was 0.89 for the ADIS-C and 0.83 for the ADIS-P.

\section{Procedure}

All children and parents received and signed an informed consent. Clinically anxious children were assessed before treatment and families from the general population were measured once. The ADIS interviews with parents and child were conducted by a research assistant. Furthermore, all family members filled out a battery of questionnaires, including the CATS. All children were assisted by a research assistant if necessary.

\section{Analysis}

Missing data were predicted using the missing value analysis of SPSS based on regression models using available data of the group mean, the available data of the individual and the pattern of change of the group. Differences on the total CATS score were calculated by ANCOVA. To measure differences on the subscales, a MANCOVA was carried out. Discriminant analyses were carried out to investigate content specificity within anxiety disorders.

\section{Results}

\section{Internal consistency}

Firstly, the internal consistency was calculated using the total sample of 200 children and adolescents. The Cronbach's alpha coefficient for the total CATS score was .94 in the total sample, which indicates a high degree of homogeneity. The internal consistencies for the subscales were also high, with alphas of .85 for physical threat, .90 for social threat, .92 for personal failure and .82 for hostile intent.

\section{Clinical versus control}

To test whether the clinical and control group differed, an ANCOVA was carried out, with a correction for gender and age. It was revealed that clinically anxious children reported significantly more negative automatic thoughts than 
children from the general population, $F(1,196)=10.5, p<.01$, pooled effectsize $=$ 0.6. Means can be found in Table 2 .

Table 2 Mean CATS Scores and Standard Deviations in the clinical $(n=139)$ and control sample $(n$ $=61)$

\begin{tabular}{|c|c|c|c|c|c|}
\hline & \multicolumn{2}{|c|}{ Clinical sample } & \multicolumn{2}{|c|}{ Control sample } & \multirow[b]{2}{*}{ F } \\
\hline & $M$ & $S D$ & $M$ & $S D$ & \\
\hline Physical threat & 5.8 & 6.7 & 2.4 & 3.4 & $12.9^{*}$ \\
\hline Social threat & 8.6 & 7.9 & 4.2 & 4.1 & $14.8^{*}$ \\
\hline Personal failure & 8.6 & 8.2 & 3.4 & 4.3 & $10.3^{*}$ \\
\hline Hostile intent & 7.7 & 6.9 & 7.9 & 5.9 & 0.0 \\
\hline Total CATS score & 29.3 & 24.2 & 18.0 & 13.6 & $10.5^{*}$ \\
\hline
\end{tabular}

Note: ${ }^{*}=p<0.01$

A MANCOVA with the four subscales as dependent variables, revealed a main effect for group as well, $F_{\text {Hotelling }}(4,193)=6.4, p<.01$. Follow-up ANOVAs showed that the clinically anxious group reported more thoughts on physical threat, $F(1,196)=12.9, p<.01$, pooled effectsize $=0.7$, social threat, $F(1$, $196)=14.8, p<.01$, pooled effectsize $=0.7$ and personal failure, $F(1,196)=10.3, p$ $<.01$, pooled effectsize $=0.8$ compared to children from the general population but no difference on hostile intent occurred, $F(1,196)=0.0$ (see Table 2).

\section{Gender effect}

An ANCOVA was carried out, to test whether gender influenced the total score of negative automatic thoughts, corrected for group and age. No main effect for gender was found, $F(1,196)=0.5, p>.1$. Also, no interaction effects with group, $F(1,192)=0.2, p>.1$ or age were found, $F(1,192)=0.3, p>.1$. Means can be found in Table 3.

However, across the four subscales, gender did influence the degree of negative automatic thoughts, $F_{\text {Hotelling }}(4,193)=3.9, p<.01$. Girls reported a significant higher degree of negative automatic thoughts related to social threat compared to boys, $F(1,196)=4.1, p<.05$, whereas boys report more negative automatic thoughts on hostile intent than girls, $F(1,196)=3.9 p=.05$. No significant difference between boys and girls was found on the physical threat subscale, $F(1,196)=1.1, p>.1$ and on personal failure, $F(1,196)=1.3, p>.1$ (see Table 3). Furthermore, no significant group by gender and age by gender interaction effects occurred, respectively $F_{\text {Hotelling }}(4,189)=0.5, p>.1$ and $F_{\text {Hotelling }}(4,189)=0.6, p>.1$ (see Table 3$)$. 
Table 3 Mean CATS Scores and Standard Deviations for boys $(n=90)$ and girls $(n=110)$

\begin{tabular}{lcrrrrl}
\hline & \multicolumn{2}{c}{ Boys } & & \multicolumn{2}{c}{ Girls } & \\
\cline { 2 - 3 } \cline { 5 - 6 } & $M$ & $S D$ & & & $S D$ & \multicolumn{1}{c}{} \\
\hline Physical threat & 4.0 & 5.5 & & 5.3 & 6.5 & 4.1 \\
Social threat & 5.4 & 5.4 & & 8.8 & 8.2 & 1.3 \\
Personal failure & 4.7 & 6.3 & & 7.2 & 8.1 & $3.9^{* *}$ \\
Hostile intent & 8.9 & 6.7 & & 6.8 & 6.5 & 0.5 \\
Total CATS score & 23.0 & 19.1 & & 28.1 & 24.2 & \\
\hline
\end{tabular}

Note: ${ }^{* *}=p<0.05$

\section{Age effect}

In order to examine age effects corrected for gender and group, an ANCOVA was carried out with age categorised into 3 age groups, namely 8-11, 12-14 and 15-18 year-olds. A significant age effect corrected for gender and group was found, $F(1,196)=6.2, p<.05$. Further analysis between the 3 age groups showed that only 15-18 year-olds reported significantly higher levels of automatic thoughts compared to 8-11, $F(1,128)=9.2, p<.01$ and $12-14$ yearolds, $F(1,117)=19.2, p<.01$. The 8-11 and 12-14 year-olds did not differ significantly, $F(1,149)=2.6, p>.05$. Again, no interaction effect with group, $F$ $(1,192)=0.1, p>.1$ was found. Means can be found in Table 4 .

Table 4 Mean CATS Scores and Standard Deviations for age 8-11 $(n=81), 12-14(n=70)$ and 15-18 $(n$ $=49$ )

\begin{tabular}{|c|c|c|c|c|c|c|c|}
\hline & \multicolumn{2}{|c|}{ 8-11 year old } & \multicolumn{2}{|c|}{$\underline{12-14 \text { year old }}$} & \multicolumn{2}{|c|}{ 15-18 year old } & \multirow[b]{2}{*}{$F$} \\
\hline & $\bar{M}$ & $\overline{S D}$ & $\bar{M}$ & $S D$ & $\bar{M}$ & $\overline{S D}$ & \\
\hline Physical threat & 5.4 & 6.5 & 2.5 & 3.4 & 6.9 & 7.3 & 0.1 \\
\hline Social threat & 5.2 & 5.3 & 6.6 & 6.5 & 11.6 & 9.1 & $19.9^{*}$ \\
\hline Personal failure & 4.9 & 6.2 & 4.1 & 4.7 & 10.0 & 1.4 & $13.6^{*}$ \\
\hline Hostile intent & 8.9 & 6.9 & 6.4 & 6.0 & 7.9 & 6.8 & 0.4 \\
\hline Total score & 24.3 & 20.3 & 19.5 & 15.7 & 37.3 & 28.2 & $6.2^{* *}$ \\
\hline
\end{tabular}

Note: ${ }^{*}=p<0.01$ and ${ }^{* *}=p<0.05$

Across the four subscales, a significant age effect was found, $F(4,193)=$ $8.1, p<.01$. Children aged 15 to 18 , reported more negative thoughts concerning social threat, $F(1,196)=19.9, p<.01$ and personal failure, $F(1,196)=13.6, p<$ .01 but not concerning physical threat, $F(1,196)=0.1, p>.1$ and hostile intent, $F(1,196)=0.4, p>.05$. Both the subscales social threat and personal failure showed a linear pattern; scores increase as age increases (see Table 4). Furthermore, no significant group by age interaction effect was revealed, $F_{\text {Hotelling }}(8,372)=0.1, p>.05$. 
Are different anxiety disorders associated with different cognitions?

In order to investigate whether having a specific anxiety disorder can be predicted by having automatic thoughts concerning a specific subscale, a discriminant analysis was carried out. The primary diagnosis was used, since this diagnosis caused the most interference with daily functioning and will therefore be associated with more frequent automatic thoughts related to that anxiety. In line with the hypotheses, separation anxiety disorder $(n=34)$ was best predicted by the physical threat subscale with a standardised canonical discriminant function coefficient (scdfc) of 1.10, followed by social threat (scdfc $=-.62)$, personal failure $(\mathrm{scdfc}=-.40)$ and hostile intent $(\operatorname{scdfc}=.28)$, Wilks' Lambda $=.90, p<.01$. Also in line with expectations, social phobia $(n=46)$ was best predicted by the social threat subscale of the CATS with a standardised canonical discriminant function coefficient of 1.35 , followed by physical threat $(\mathrm{scdfc}=-.72)$, personal failure $(\mathrm{scdfc}=-.42)$ and hostile intent $(\mathrm{scdfc}=-.21)$, Wilks' Lambda $=.84, p<.01$. In contrast to our hypotheses, specific phobia $(n=$ $24)$ and panic disorder $(n=11)$ were not predicted by the physical threat subscale, respectively Wilks' Lambda $=.97, p>.05$ and Wilks' Lambda $=.94, p>$ .05 . In line with expectations, generalised anxiety disorder $(n=24)$ was not predicted by a specific subscale, Wilks' Lambda $=.97, p>.05$.

Do children with comorbid depression have more thoughts on personal failure?

To investigate the hypothesis that clinically anxious children with comorbid depression have a significantly higher score on the CATS subscale personal failure, the group of clinically anxious children was divided into a group without depressive disorder and/or dysthymic disorder $(n=113)$ and a group with depressive disorder and/or dysthymic disorder $(n=26)$. Children with depression and/or dysthymic disorder $(M=11.7, S D=10.4)$ reported significantly more negative automatic thoughts on personal failure than children without depression and/or dysthymic disorder $(M=6.2, S D=7.2)$,

$F(1,137)=10.2, p<.01$. Children with depression and/or dysthymic disorder $(M=11.8, S D=9.4)$ also reported significantly higher levels of automatic thoughts on social threat than children without depression and/or dysthymic disorder $(M=7.8, S D=7.4), F(1,137)=5.6, p<.05$. No difference was found on physical threat, $F(1,137)=0.0, p>.05$ and hostile intent, $F(1,137)=0.2, p>.01$. The discriminant analysis showed that depression was predicted by high scores on personal failure $(\mathrm{scdfc}=1.44)$, followed by physical threat $(\operatorname{scdfc}=-.98)$, social threat $(\mathrm{scdfc}=-.10)$ and hostile intent $(\operatorname{scdfc}=.03)$, Wilks' Lambda $=.87$, $p<.01$.

\section{Discussion}

The aim of this study was to investigate the utility of the CATS to measure negative automatic thoughts in clinically anxious and control youths. The main results can be summarised as follows. The first finding of this study is that, in 
line with our hypothesis and Schniering and Rapee (2002), clinically anxious children reported more negative automatic thoughts compared to control children on the total score and on the subscales physical threat, social threat and personal failure. Secondly, girls reported more negative automatic thoughts on the subscale social threat, whereas boys reported more thoughts concerning hostile intent. Thirdly, 15-18 year-olds reported the highest frequency of negative automatic thoughts, compared to 8-11 and 12-14 year-olds. The latter two did not differ. Fourthly, children with separation anxiety reported more thoughts concerning physical threat, while children with social phobia as primary anxiety disorder reported more thoughts on social threat. Fifthly, anxious children with comorbid depression had higher scores on personal failure than children without comorbid depression.

The finding that anxious children reported a higher frequency of negative anxiety related thoughts (total CATS score) is in line with different studies, some using the same methodology to measure cognitions for example by means of self report (Ronan, et al., 1988), and some using different methodology like interpretations of ambiguous situations (Bögels \& Zigterman, 2000).

On subscale level, clinically anxious children report more negative automatic thoughts concerning physical threat and social threat compared to control children. This finding is consistent with Beck's cognitive specificity model, which states that threat is more related to anxiety (Beck et al, 1987; Beck \& Clark, 1988). No difference on the hostile intent subscale was found, which is also consistent with the cognitive specificity model. Hostile intent is more associated with externalising disorders.

In line with the hypothesis and the findings of Schniering and Rapee (2002), high scores on the personal failure subscale in the clinically anxious group were found. This can be explained by the fact that thoughts on personal failure might not be specific for depression. Marien and Bell (2004) suggest a cognitive overlap between anxiety and depression, which is called negative cognitivity. Negative cognitivity is a general negative cognitive bias characterised by negative thoughts like negative self-evaluation, prediction of negative outcomes, and a negative interpretation bias. Also a study by Jolly (1993) showed that anxious cognitive content correlated to clinician and selfreport anxiety symptoms but also correlated to self-reported depressive symptoms.

Jolly and Dykman (1994) found evidence for a more cognitive level of the tripartite model, in which a general distress factor is considered as a shared feature for depression and anxiety but also anxiety and depression are seen as having unique characteristics (Clark \& Watson, 1991). In adolescents, anxious cognitions were associated with anxiety, depressive cognitions were associated with depression and both were also associated with more general cognitions. Ambrose and Rholes (1993) showed that the severity of anxious cognitions have an influence on the overlap of depressive cognitions in anxious children. At 
some level of severity, threat cognitions encourage depressive symptoms and the association of anxious cognitions and anxious symptoms becomes less strong. Hence, in children with high threat cognitions also depression-related cognitions are prevalent. Even more, $19 \%$ of the clinically anxious children had comorbid depression, which might cause the increase in negative automatic thoughts concerning personal failure in anxious children.

Girls had higher scores on social threat, while boys reported more negative thoughts on hostile intent. This could be due to gender role stereotyping, in which boys are not culturally allowed to report anxious thoughts. A study of Pierce and Kirkpatrick (1992) showed that when male students are given instructions that their truthfulness could be measured by means of changes in heart rate, their fears on a fear schedule increased while no such increase was found in female students. This implies that males as do boys fill in the questionnaire in a cultural desirable way. Girls are also more concerned with others' opinions of them because they want to be "liked" (Rosenberg \& Simmons, 1975) thereby they might have more negative thoughts on the social threat subscale. This is also in line with Campbell and Rapee (1994), who showed that social threat thoughts were more prevalent in girls. Furthermore, internalising problems are more prevalent in girls, while externalising problems are more prevalent in boys (Verhulst et al., 1997), which might result in more corresponding cognitions of respectively social threat and hostile intent. In contrast to Schniering and Rapee (2002) no difference on the personal failure subscale was found between boys and girls. In conclusion, there are gender differences in thoughts of clinically anxious children.

The finding that 15-18 year-olds report more negative automatic thoughts than 8-14 year-olds on the total CATS score, social threat, and personal failure can be explained by Piaget's theory. Piaget (1987) proposed three different stages of reasoning. First, the preoperational stage (1,5-7 year-olds), in which children generate a limited number of possible solutions. Second, the concrete operational stage (7-11 year-olds), in which children are able to generate several possibilities and are able to perform deductive reasoning. Third, the formal operation stage ( $>11$ year-olds), in which children are able to anticipate an unlimited number of possibilities and perform deductive reasoning. Thus, with increasing age, the child's cognitive skills develop and the child will form more possible outcomes, increasing the risk of having more negative cognitions. The older children also reported more negative thoughts on personal failure. This is in line with Brady and Kendall (1992) who showed that younger children often solely have anxiety, while older children have anxiety with comorbid depression. The fact that social threat cognitions increased, is in line with the fact that the onset of social anxiety commonly occurs in early to mid adolescence (Bernstein, Borchardt, \& Perwien, 1996). Due to the cognitive development of anxious children, thoughts increase in number and severity. 
Some evidence for diagnostic specificity of the type of negative automatic thoughts within specific anxiety disorders was found. Children with separation anxiety disorder and social phobia had higher scores on respectively physical threat and social threat than children without these primary diagnoses. These two findings are consistent with the corresponding DSM-IV criteria of the diagnosis (APA, 1994). The fact that we did find evidence for the specificity of negative thoughts in children with social phobia and children with separation anxiety might suggest that the effectiveness of cognitive behaviour therapy can be enhanced by targeting these specific thoughts when the child is diagnosed with separation anxiety or social phobia. Contrary to our hypothesis, we did not find that children with specific phobia and panic disorder had more negative thoughts on physical threat compared to children without those disorders. As for children with generalised anxiety disorder we did not find higher scores on all the subscales compared to children without generalised anxiety disorder. This is in line with the study by Bögels et al. (2003), who found that children with generalised anxiety symptoms did not report more interpretations consistent with the content of their fear.

Clinically anxious children with comorbid depression had a higher score on the subscale personal failure. Several studies show that depressed children have more thoughts related to personal failure than non-depressed children (Kaslow, Stark, Printz, Livingston \& Tsai, 1992). The latter finding is also consistent with Beck's cognitive specificity model (Beck et al., 1987; Beck \& Clark, 1988) and other studies showing that depression is associated with cognitions concerning personal failure (Ambrose \& Rholes, 1993; Jolly 1993; Marien \& Bell, 2004). The higher frequency of social threat thoughts can be explained by the fact that depressive children often withdraw from peers, thereby increasing anxiety to interact with peers (de Wit, 2000). Taken together, this research and previous literature shows that children with (comorbid) depression have more personal failure thoughts.

The limitations of this study are that we did not include a sample of children with externalising disorders, nor did we include children with depression as primary diagnosis. Furthermore, this study was cross sectional instead of longitudinal. When anxious and non-anxious children are followed throughout their development on their automatic thoughts related to threat, one might find different results.

The finding that negative automatic thoughts are more prevalent in clinical children stresses the importance of changing negative cognitions during treatment. Mifsud and Rapee (2005) already demonstrated that a significant change on the CATS was found after a school based cognitive intervention. Future studies should assess cognitions in effectiveness studies as well, to not only compare effects related to anxiety but also related to cognitions. 


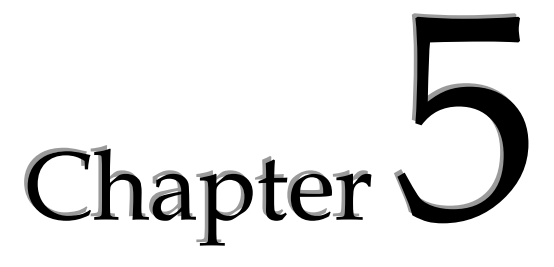

Cognitive Behavioural Therapy in children with anxiety disorders; an overview

Denise H. M. Bodden 


\section{Introduction}

Anxiety disorders are the most prevalent disorders in children, cause interference with daily functioning of the child and if untreated the prognosis of an anxiety disorder is quite poor (Keller, et al., 1992). Of 725 children aged 6 to 19 year-olds, $14 \%$ were found to have an anxiety disorder (past or present), of which $66 \%$ still had an anxiety disorder after approximately 4 years duration (Keller et al., 1992). Moreover, other research has shown that children with anxiety disorders still met diagnostic criteria up to 8 years after the onset of the anxiety disorder (Kovacs \& Devlin, 1998).

Diverse treatments for children with anxiety disorders exist for instance pharmacotherapy, Cognitive Behavioural Therapy (CBT), Psychodynamic Therapy, Eye Movement Desensitisation and Reprocessing (EMDR) and Relaxation Therapy. However, Cognitive Behavioural Therapy (CBT) is the only evidence-based treatment in children with anxiety disorders. This chapter contains a review regarding CBT throughout the last twenty years. The components, the history and the diverse variations of CBT will be discussed. Even more, a table with randomised control trials will be provided.

In the last quarter of this century, a substantial number of randomised control trials were conducted to study the effectiveness of CBT. CBT includes a range of therapeutic techniques based on the principle that thoughts, feelings and behaviour are closely related. CBT includes a combination of different techniques to recognise and alter distorted thoughts that anxious children and adolescents experience prior to or in a feared situation. It is assumed that these distorted cognitions (overestimation of negative events or danger and inability to cope with threat) lead to anxiety and maladaptive behaviour like avoidance. By modifying these cognitions, behaviour will change as well. Furthermore, CBT consists of techniques to change behaviour. The most commonly used technique is exposure in vivo. This real life exposure consists of approaching the feared stimuli or situation gradually from least to most fearful. So far, CBT is proven to be the only efficacious treatment for children with anxiety disorders.

\section{Components of CBT}

CBT for children with anxiety disorder has basically 5 components; psycho education, somatic management skills, cognitive restructuring, exposure and relapse prevention. Psycho education provides information about anxiety and the feared stimuli. Somatic management provides techniques to reduce autonomic arousal and related physiological responses by making the child aware of its reaction to anxiety and to control these reactions. Furthermore, relaxation training, often a combination of progressive muscle relaxation, deep breathing and cognitive imagery, is used.

Cognitive restructuring focuses on identifying maladaptive thoughts and teaching the child a more coping focused thinking. Behaviour experiments 
can help to challenge maladaptive thoughts. In behavioural experiments, evidence is collected as a support for or against a certain hypothesis the child has concerning its anxiety. Exposure involves a gradual approach towards a feared stimuli or situation so anxiety can decrease, this is called extinction. Through recurrent exposure, the link between the feared stimulus and the anxiety reaction will weaken. Often an anxiety hierarchy is generated with anxious situations or stimuli that gradually increase in severity from low to most fearful.

Relapse prevention methods provide consolidating and generalising principles to maintain treatment effects over time. Furthermore, a commonly used technique in CBT is modelling. Modelling involves demonstrating courageous behaviour in a fearful situation so the child can imitate this desired coping behaviour in reaction to its own anxiety.

\section{Pioneers in $\mathrm{CBT}$}

In contrary to adult anxiety literature, treatment studies involving children with anxiety disorders were not conducted until late in the $20^{\text {th }}$ century. In 1988, King, Hamilton and Ollendick concluded that behavioural techniques were effective in specific childhood fears and phobias. A year later, Kane and Kendall (1989) conducted a pilot study in which 16 to 20 sessions CBT were given to 4 children aged 9 to13 with overanxious disorder. Both post treatment as followup data showed a decline on parent, clinician and child report questionnaires.

Dadds, Heard and Rapee (1992) were the first to report preliminary results on a treatment with parental involvement. In their study, a 12-session family anxiety management training was given parallel to child CBT. Children aged 7 to 14 with overanxious disorder $(n=10)$ and separation anxiety disorder $(n=4)$ were randomised to CBT with family management $(n=7)$ or the waitlist condition $(n=7)$. Child CBT with family anxiety management was found to be more effective ( $70 \%$ free of anxiety disorders) than the waitlist condition $(0 \%$ free of anxiety disorders) (Dadds et al., 1992).

From this point on, diverse protocols were developed and randomised control trials were conducted for children with anxiety disorders. CBT was given in individual (child alone) CBT format or group CBT format. Additionally family components were included throughout the development of CBT for anxiety disorders in children. This family component was given parallel to child CBT. Recently, family CBT was developed involving both children and parents simultaneously in treatment. Outcome measures usually reflect the percentage of children free from any anxiety diagnosis (adf) or the percentage of children free from their primary anxiety diagnosis or the most intervening diagnosis (pdf). An overview of these RCT's can be found in Table 1.

\section{Individual CBT}

Kendall was the first to develop a CBT program for children with anxiety disorders namely Coping cat (Kendall, Kane, Howard, \& Siqueland, 1990). This 
program involves 16 to 20,60-minute weekly sessions for children aged 7 to 13 . The program consists of a treatment manual for the therapist and a workbook for the child. Both are developed especially for children with a primary diagnosis of separation anxiety disorder, generalised anxiety disorder or social phobia. The main principles are recognising anxious feelings and physiological reactions to anxiety, identifying and challenging cognitions in anxietyprovoking situations, developing coping strategies with the fearful situation (coping self-talk), exposure and evaluating performance. The FEAR acronym is used; F-Feeling frightened?; E-Expecting bad things to happen; A-Actions and attitudes that help; and R-Results and rewards (Kendall et al., 1990).

Kendall was also the first to publish data on Individual CBT. His study showed that Individual CBT (64\% pdf) is more effective compared to waitlist (5\% pdf) (Kendall, 1994). Barrett adapted Kendall's coping cat into Coping Koala (Barrett, Dadds \& Rapee, 1996), which focuses more on exposure and cognitive restructuring. This adapted version was also effective in treating children with anxiety disorders with percentages ranging from $57 \%$ (post treatment) to $71 \%$ (1 year follow-up) free of anxiety diagnosis (Barrett et al., 1996). Even more, this protocol has been translated in other languages, including a Dutch protocol called "De dappere kat" by Nauta, Scholing, Emmelkamp and Minderaa (2001).

Another individualised CBT is Social Effect Therapy (SET-C; Beidel, Turner, \& Morris, 2000), which was developed specifically for children with social phobia. The treatment involves reduction of social anxiety, improvement of social skills and an increase of participation in social activities. The components consist of education, social skills training, peer generalisation experiences (practice acquired social skills in different natural settings) and exposure in vivo. SET-C combines child alone and group sessions. This treatment was proven to be effective ( $67 \%$ pdf), even up to 6 months follow-up (85\% pdf) (Beidel et al., 2000).

\section{Individual CBT with parents}

In 1996, Howard and Kendall extended the Coping Cat protocol to a family based CBT. In a pilot study with 6 children with overanxious disorder and separation anxiety disorder, it was found that this treatment was effective, indicated by the fact that $83 \%$ did not fulfil criteria of any anxiety diagnosis (Howard \& Kendall, 1996).

Barrett et al. (1996) tested the additive efficacy of additional family anxiety management combined with the Coping Koala protocol for child CBT (Heard, Dadds, \& Rapee, 1991 in Barrett et al., 1996). Family anxiety management involves education of the parents in contingency management to reinforce coping and courageous behaviour and ignore anxious behaviour, it teaches parents to cope with their own anxiety and model positive or adaptive coping in stressful situations (management of own anxiety), and parents receive a training in communication and problem solving skills, so they are better able to work as a team. This intervention consists of 12 sessions and can be given in 
a parent group format (Barrett, 1998) or a combination session with first, the child alone, followed by child and parents together. At post treatment individual CBT combined with family management $(84 \%$ adf) was more effective than individual CBT alone (57\% adf). However, at 6 months follow-up this effect disappeared. Again, at 1-year follow-up, an additive value of individual CBT with family management was shown (Barrett et al., 1996).

Besides working with parents, sometimes teachers are involved. A Parent and teacher training (King et al, 1998) was given to parents of children with school refusal. It involves parent sessions and school personnel consultation. Parents receive 5 sessions in behaviour management strategies focussed on school attendance, are taught to use positive reinforcement and received cognitive therapy. School personal consultation consisted of discussing the treatment plan, facilitating the child's return and teaching positive reinforcement with school personnel (King et al., 1998). Two studies of King et al. (1998 and 1999) have shown that individual CBT with parent/teacher training was effective in the percentage of children with school refusal meeting the criterion of $90 \%$ school attendance.

Exposure based contingency management (Silverman, Kurtines, Weems, et al., 1999) was developed to facilitate exposure by using contingency management techniques for parents. Parents were taught behavioural strategies like positive reinforcement and reward to help the child with the exposure. Contingency management (55\% adf) was found to be as effective as a control educational support (56\% adf) and less effective as self control exposure $(88 \%$ adf) (Silverman, Kurtines, Weems, et al., 1999).

Nauta et al. (2001) developed a 7-session Cognitive parenting program focusing on parents' behaviour, thoughts and feelings regarding their anxious child. This program was given additionally to child CBT. Sessions consisted of providing psycho education, behavioural advice and parenting skills, problem solving skills and targeting parents' thoughts about their anxious child. No additive value of this parenting program was found (Nauta et al. 2001; Nauta, Scholing, Emmelkamp, \& Minderaa, 2003).

Not only protocols but also books are used in treatment studies. The book Keys to parenting your anxious child (Manassis, 1996 in Manassis et al., 2002) is used in different studies using a group therapy for parents. It contains strategies to educate and help parents cope with their child's anxiety (Manassis et al., 2002).

\section{Individual CBT with anxious parents}

Cobham, Dadds and Spence (1998) conducted a study to investigate whether individual CBT with parental involvement is more effective when parents are anxious themselves. Parents had to have a State Trait Anxiety Inventory (STAI: Spielberger, 1973) score of 40 or higher to be labelled as anxious. They developed an intervention named Parental anxiety management (Cobham et al., 1998). This intervention consists of 4 parent sessions in combination with 10 
sessions of child CBT (Coping Koala). The sessions involved psycho education, cognitive restructuring, relaxation training, and contingence management. It was found that in the short term, individual CBT with parental involvement was more effective when parents were anxious themselves. At follow-up the additive value of parental involvement disappeared. Children with nonanxious parents were treated equally well with individual CBT or individual CBT with parental involvement both at short term as at long term follow-up (Cobham et al., 1998).

\section{Group CBT}

Barrett adapted and extended Kandall's Coping Cat into a group format of Coping Koala (Barrett, 1998). This format showed to be effective at post treatment and 1-year follow-up (respectively 56\% and 6\% \% adf). Another group format was developed by Flannery-Schroeder and Kendall (2000) using the Coping Cat protocol. At post treatment, group CBT (50\% pdf) was not significantly more effective than individual CBT (73\% pdf). Also, at 3-month follow-up, the percentage children free of their primary anxiety disorder did not differ significantly, group CBT 53\% and individual CBT 79\% (FlannerySchroeder \& Kendall, 2000).

\section{Group CBT with parents}

Barrett (1998) tested the additive efficacy of additional family anxiety management combined with the Coping Koala group version. Group CBT with family management was equally effective as solely group CBT both at post treatment and at 1-year follow-up (Barrett, 1998). Another group version of the Coping Cat workbook was developed namely, the Coping Bear workbook (Manassis, Avery, Butalia, \& Mendlowitz, 2004).

Spence, Donovan, and Brechman-Toussaint (2000) specifically developed a group CBT with parental involvement for children with social phobia. The program involves social skills training, relaxation techniques, social problem solving, positive self-instruction, cognitive challenging and exposure (social skills practice in "games" session). Parental involvement consisted of modelling, reinforcing social skills and encouraging the child to socially interact. Parents observed the child's group session through a one-way screen. Furthermore, parents received handouts and group training sessions. Group CBT with parental involvement ( $88 \%$ pdf) was more effective than solely group CBT ( $58 \%$ pdf) at post treatment. At 1-year follow-up, group CBT with parental involvement (88\%) was trend significant more effective than group CBT (53\%), indicating an almost significant finding (Spence et al., 2000).

In 2000, Barrett and colleagues further refined family based group CBT, which was called Friends (Barrett, Lowry-Webster, \& Turner, 2000 in Shortt, Barrett, \& Fox,. 2001). Friends is a acronym for strategies which are taught; FFeeling worried?; R- Relax and feel good; I-Inner thoughts/I can do it; EExplore solutions and coping plans; N-Nice work so reward yourself; D-Don't 
forget to practice; and S-Stay calm, you know how to cope now. The Friends program also teaches children to think of their body as friend (the body shows the child when it's anxious), to be their own friend (try hard and reward themselves), to make friends (social support) and to talk to their friends in difficult situations. There are two developmentally sensitive versions; one for children aged 6 to 11 and one for youth aged 12 to 16 . The parents are involved in a group format by teaching them cognitive restructuring and to cope with their own anxiety, reinforcement strategies, partner support training and to engage in social activities. This parent training can be given either in $4,1 \frac{1}{2}-$ hour sessions or 10 sessions of 40 minutes parallel to the child sessions. The Friends program with parental involvement was effective; $69 \%$ of the children was free of all their anxieties at post treatment. At 1-year follow-up, this percentage was $68 \%$ (Short et al., 2001). Currently, a study is investigating the effectiveness of the FRIENDS program in Dutch children. Results have not been published yet (Treffers, Ferdinand, Van Widenfelt, Utens, Liber \& van der Leeden, personal communication).

Facing your fears (Cobham, 2003) is another group CBT program, which consists of 6 sessions. Both child and parent are present in the beginning and end of the sessions. This program contains of two steps namely helpful thoughts and brave behaviours. This program was given specifically to nonanxious parents. At post treatment, no child was free of its anxiety disorder. At 3-month follow-up, $80 \%$ was free of their anxiety disorders. This percentage even climbed to $100 \%$ (adf) at 1-year follow-up (Cobham, 2003).

Family CBT

Two studies did include the parents in the child sessions, so the child and parents were simultaneously involved in the treatment. The Attachment based family therapy of Siqueland, Rynn and Diamond (2005) focuses on family interaction like parental beliefs about anxiety, overprotection and psychological control. The main goal of therapy is to encourage his or her autonomy. Three to four sessions are alone with the adolescent to establish the child's autonomy. Parents are taught CBT skills to help their child and not to cope with their own fear. This treatment was not found to be more effective than individual CBT alone (Siqueland et al., 2005).

In a study of Wood, Piacentini, Southam-Gerow, Chu, and Sigman (2006) a family based CBT program named the Building confidence program was used. This therapy focuses on parental communication, more specifically intrusiveness and autonomy granting. This program combines child CBT techniques like skills training and exposure with parent training. Sessions were divided in three parts; child alone (15 to 30 minutes), parents alone (25-30 minutes) and parents and child together (10 to 15 minutes). The family based building confidence program (79\%) was equally effective compared to individual CBT (53\%) at post treatment (Wood et al., 2006).

Recently, a new family CBT was developed, including both parents and siblings of anxious children (Bögels \& Siqueland, 2006). In this family CBT, 
child and parents are taught to reduce their own anxiety using CBT skills. The therapist guides the parents to be co therapists of the child by modelling courageous behaviour and guiding the exposure of the child. Dysfunctional thoughts of parents on parenting ("I have to be available to my child all the time"), the anxious child ("My child is vulnerable"), and the safety of the child's world ("The world is full of anger") are targeted. The child's thoughts concerning the parents ("I'm not allowed to bother my parents with my anxiety") and about using anxiety as means of communication are challenged. Furthermore, family interaction is modified by improving communication and problem solving with the goal of granting more autonomy to the child. Parental conflict and communication problems about handling their anxious child are also addressed. A pilot study using this family CBT showed that 59\% of the 17 children were free of their primary anxiety disorder at 3-month follow-up (Bögels \& Siqueland, 2006).

\section{Parent CBT}

Cool kids is a group CBT program solely for parents and was developed by Lyneham et al. (2003 in Thienemann, Moore, \& Tompkins, 2006). This treatment was based on Kendall's Coping Cat and the workbooks used are additional to "Helping you anxious child" (Rapee, Spence, Cobham, \& Wignall, 2000). Elements of this therapy are psycho education, parenting strategies, exposure ("stepladders"), problem solving, mindfulness techniques, and social skills training. Parents learn skills to help their child with their anxiety as lay therapists (Thienemann et al., 2006).

\section{Control conditions}

In most RCT studies, the control group consists of a waitlist group. However four studies used an active control group. The first is Educational support therapy (Last, Hansen, \& Franco, 1998), which is an attention placebo control and involves a combination of educational presentations and supportive psychotherapy. Children also have to keep a daily diary. Last et al. (1998) found that educational support $(50 \%)$ was found to be equally effective as individual CBT (65\% adf). The second, Exposure based cognitive self-control (Silverman, Kurtines, Weems, et al., 1999) is a non-active control condition in which children and parents were given education about anxiety and treatment and received therapeutic support. However, no exposure was given. Contingency management (55\% adf) was found to be as effective as educational support (56\% adf) (Silverman, Kurtines, Weems, et al., 1999).

The third, Testbusters (Beidel, et al. 2000) is an active but non-specific intervention including individual and group sessions. Testbusters is a program to develop good study-skills and test-taking strategies. Testbusters (5\% pdf) was found to be less effective than social effect therapy (67\% pdf) (Beidel et al. 2000). Fourth, Muris, Meesters, \& van Melick (2002) used emotional disclosure as a control condition. Children had to keep a diary in which they wrote down 
their thoughts and feeling concerning threatening situations. It was found that group CBT ( $80 \%$ within normal range of Revised Child Anxiety and Depression Scale; Chorpita, Yim, Moffitt, Umemoto, \& Francis, 2000) was more effective compared to emotional disclosure (40\% within normal range of RCADS) (Muris et al., 2002).

\section{Other treatments}

Some studies have investigated a specific ingredient of CBT namely exposure. In the following studies only questionnaire results were obtained, therefore they are not included in Table 1. Menzies and Clarke (1993) investigated the efficacy of 3 sessions in vivo exposure, vicarious exposure, in vivo exposure combined with vicarious exposure or waitlist. In vicarious exposure, the child observes a model (therapist) interacting in water-related activities for 15 minutes. Participants were 48 children aged 3 to 8 with water phobia. At post treatment, the combination of in vivo exposure and vicarious exposure was equally effective as in vivo exposure. However, both were more effective than vicarious exposure and waitlist. Vicarious learning was more efficacious than waitlist. At 12-week follow-up, the combination of in vivo exposure and vicarious exposure was more effective than in vivo exposure, which was more efficacious than vicarious exposure on questionnaires and a behaviour rating scale.

Cornwall, Spence, and Schotte (1996) found that a 6-session emotive imagery was more efficacious than a 20-week waitlist on self-report, parent report and a behavioural observation in children $(n=24)$ with darkness phobia, aged 7 to 10. Muris, Merckelbach, Holdrinet, and Sijsenaar (1998) compared 2,5 hours Eye Movement Desensitisation and Reprocessing (EMDR), exposure and computerised exposure. EMDR was given using the protocol of Shapiro (1995 in Muris et al., 1998) for specific phobia. The goal of EMDR is desensitisation of aversive experiences using horizontal eye movements during negative images/thoughts or feelings. The computerised CBT involved confrontation with spiders on a computer screen. Children aged 8 to 17 with spider phobia were included. After Exposure $(n=9)$, an improvement on all measures was found. Children treated with $\operatorname{EMDR}(n=9)$ showed improvement on selfreported spider fear. Computerised exposure $(n=8)$ showed a non-significant improvement. Therefore it was concluded that exposure was more efficacious than EMDR, which was more efficacious than computerised CBT. In a second phase all children received 1,5 hours exposure (Muris et al., 1998).

Ost, Svensson, Hellström, and Lindwall (2001) showed that a 1-session exposure $(n=21)$ was equally effective as exposure with the parent present (for instance as a model) $(n=20)$. Both were more effective than a waitlist condition $(n=19)$ in children with phobias, aged 7 to17 (Ost et al., 2001). 


\section{Concluding remarks}

This overview shows that CBT is effective in treating children with anxiety disorders. Not only at the short term but even up to 9 years follow-up, the percentage of children free of their primary anxiety disorder is quite high (Kendall, Safford, Flannery-Schroeder, \& Webb, 2004). Both individual CBT and group CBT are effective compared to a waitlist condition. Two studies show that individual CBT and group CBT are equally effective (Flannery-Schroeder \& Kendall, 2000; Muris, Mayer, Bartelds, Tierney, \& Bogie, 2001), whereas one study showed that individual CBT is more effective. However the latter study, only presented results based on questionnaires (Manassis et al., 2002).

With respect to parental involvement, results are more inconsistent. Individual CBT with family management was found to be more effective than individual CBT alone at post treatment and 1-year follow-up (Barrett et al., 1996) and if parents were anxious themselves (Cobham et al., 1998). However, in 4 studies no additive value of parental involvement (Nauta et al., 2001 and 2003), family attachment based therapy (Siqueland et al., 2005) or family based CBT (Wood et al., 2006) was found. Combined with group CBT, no surplus value of family management at the short term (Barrett, 1998) or long term (Barrett et al., 2001) was found. Even more combined parent and child groups were not significantly more effective than child group CBT alone (Mendlowitz et al., 1999). Spence et al. (2000) found that parental involvement with group CBT was more effective at post treatment, however at 1-year follow-up this effect was found to be borderline significant. Taken together, results suggest that the majority of studies show that parental involvement is not more effective than solely individual CBT. Correspondingly, a recent meta-analysis by In-Albon and Schneider (in press) showed almost no difference between child and family CBT. 


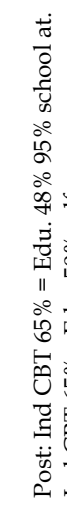

II

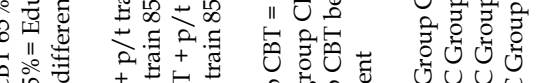

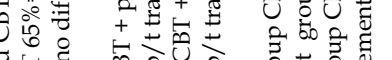

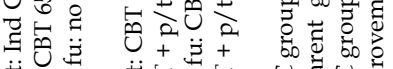

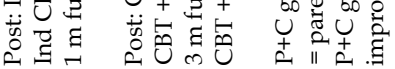

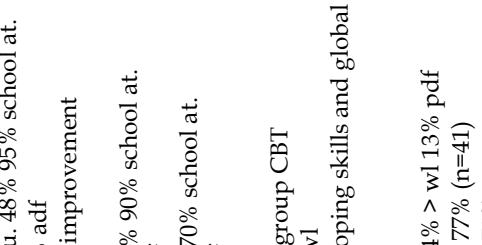

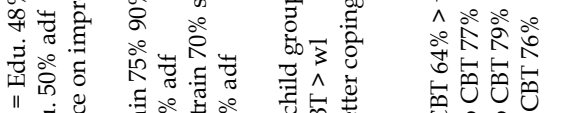

䒕 $\frac{0}{\pi}$

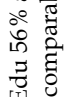

11. 先

iำ

हี :ี

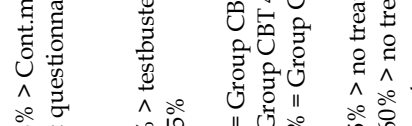

œ

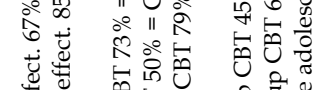

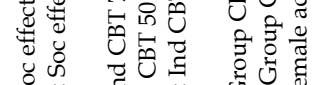

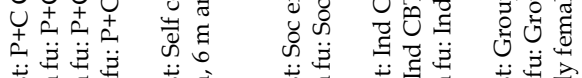

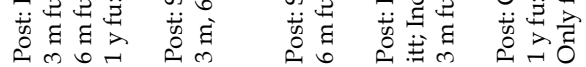

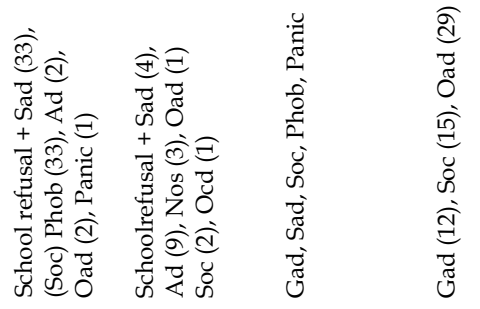

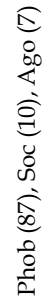

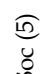

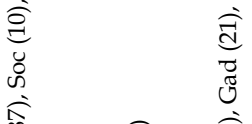

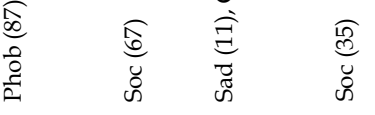

$\approx$

$\rightarrow$ เก

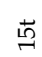

जิ

$\stackrel{2}{\mathfrak{1}}$

$\frac{0}{b}$

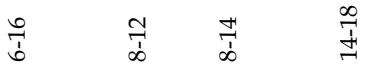

กี ูี ชิ

$\stackrel{\infty}{\rightarrow}$ 구유

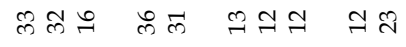

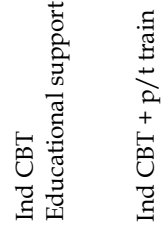

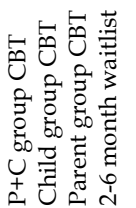

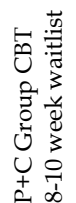

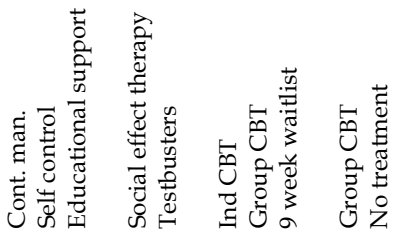

๙

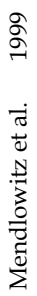

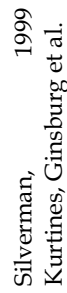

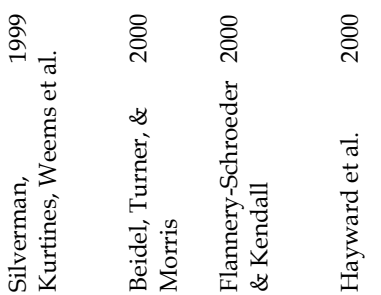


Chapter 5

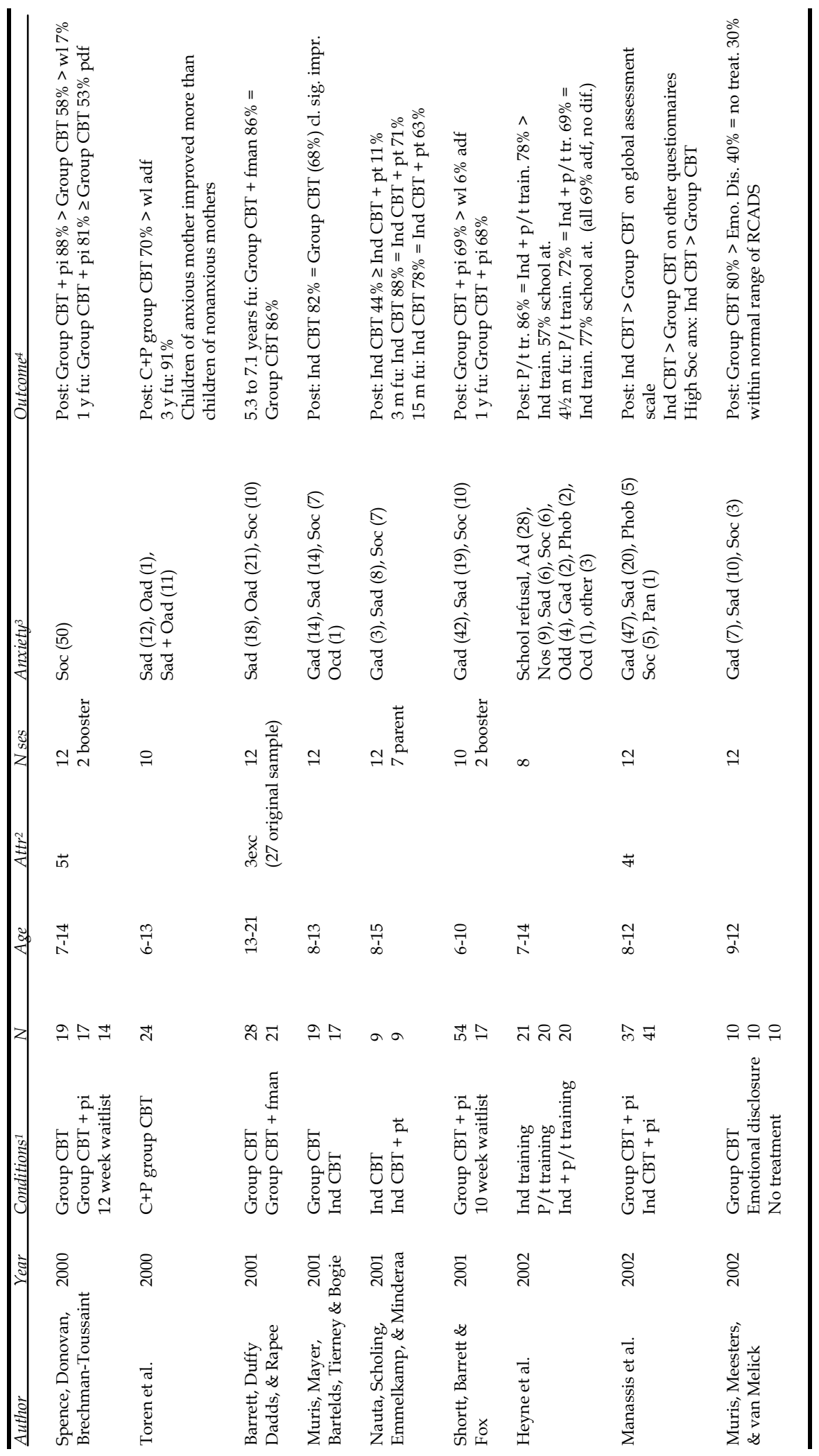









\section{Chapter}

\section{Efficacy of Individual versus Family Cognitive Behavioural Therapy in clinically anxious youth}

Denise H.M. Bodden, Susan M. Bögels, Maaike H. Nauta, Else de Haan, Jaap Ringrose, Carla Appelboom, Andries G. Brinkman, \&

Karen C.M.M.J. Appelboom-Geerts 
Abstract

The efficacy of child-focused versus family-focused Cognitive Behavioural Therapy (CBT) for clinically anxious children and adolescents was evaluated, in particular in relation to parental anxiety disorders and the child's age. Families $(n=128)$ were randomly assigned to individual or family CBT and evaluated at pre-treatment, post-treatment and 3-month follow-up. Twenty-five families were measured before and after a wait-list period. None of these wait-list children recovered from their anxiety disorders. In contrast, $41 \%$ of the children receiving treatment no longer met criteria for any anxiety disorder after treatment with continued improvement of $52 \%$ at three-month follow-up. In contrast with expectations, significantly more children were free of all anxiety disorders in individual (53\%) compared to family (28\%) CBT at post-treatment, whereas at three-month follow-up, the superior effect of individual CBT was no longer significant. Contrary to expectations, when parents themselves had anxiety disorders, family CBT was not more effective than individual CBT. Finally, family CBT was not more effective in younger and individual CBT not more beneficial in older children, as was expected. Overall, individual CBT seems slightly more effective than family CBT. 
CBT is an efficacious treatment for children and adolescents with anxiety disorders. A systemic review of 22 randomised controlled trials (RCT) by Cartwright-Hatton, Roberts, Chitsabesan, Fothergill, \& Harrington (2004) found an overall remission rate of $56.5 \%$ for CBT and $34.8 \%$ for control conditions. Similar results were found in a review of James, Soler, \& Weatherall (2005), 56\% for CBT and $28 \%$ for controls. A meta-analysis showed mean overall treatment effect sizes of $d=.84$ for CBT versus $d=.13$ for waitlist (In-Albon \& Schneider, in press). Following treatment, $70.4 \%$ of children were free of anxiety disorders, compared to $14 \%$ after waitlist.

Anxiety disorders run in families. In addition to genetic factors, environmental factors such as anxious modelling, parental conflict, maladaptive family functioning, parenting styles and parental beliefs are associated with child anxiety (Bögels, \& Brechman-Toussaint, in press). This suggests that, although the exact nature of transmission is not well understood, parents should be involved in their anxious child's treatment.

However, evidence for the additive value of parental involvement is inconsistent. Barrett, Dadds, \& Rapee (1996) found that individual CBT with additional family management was more effective than CBT alone. However, group CBT plus family management was not superior (Barrett, 1998). Similarly, children with social phobia benefited more from group CBT plus parent training than group CBT alone, but at 12-month follow-up this effect was no longer significant (Spence, Donovan, \& Brechman-Toussaint, 2000). Other group CBT studies (Barrett, Duffy, Dadds, \& Rapee, 2001; Mendlowitz, et al., 1999) as well as other individual CBT studies (Nauta, Scholing, Emmelkamp, \& Minderaa, 2001; Nauta, Scholing, Emmelkamp, \& Minderaa, 2003) did not show a superior effect of family involvement. A recent study comparing familyfocused CBT with child-focused CBT did find family CBT to have an additional benefit based on questionnaire results, however the percentage of anxiety free children did not differ significantly between family CBT (79\%) and individual CBT (53\%) (Wood, Piacentini, Southam-Gerow, Chu \& Sigman, 2006). Correspondingly, In-Albon and Schneider (in press) reported almost no difference between child and family CBT in their meta-analyses.

Parental anxiety can cause or maintain child anxiety by reinforcing avoidance, anxious modelling, and confirmation of threat interpretations (Ginsburg, \& Schlossberg, 2002). Therefore, parental involvement seems most important when parents are anxious themselves. Indeed, parental anxiety management enhanced the efficacy of CBT for anxious children if one of the parents had high trait anxiety (Cobham, Dadds, \& Spence, 1998). Furthermore, phobic children benefited more from treatment with modelling by the mother if the mother was treated as well, but only if the mother was phobic (Windheuser, 1977). These studies suggest that if parents are anxious themselves, parental involvement may enhance CBT efficacy. 
The age of the child is another relevant issue in considering efficacy of child versus family CBT. Family factors are more influential for young children, whereas older children are more autonomy seeking, which reduces family influence and increases the influence of peers (Hudson, Kendall, Coles, Robin, $\&$ Webb, 2002). Therefore, older children might benefit more from individual CBT, and young children more from family CBT. This idea was supported by Barrett et al. (1996), who found a higher percentage of children aged 7-10, free of any diagnosis after CBT plus family management $(100 \%)$ than after CBT alone $(56 \%)$, whereas in children aged 11 to 14 no difference was found (both $60 \%$ free of any disorder). Hence, this study suggests family CBT to be more beneficial in younger children.

Several factors may have contributed to lack of support for the hypothesized additive effect of family CBT. First, most studies have involved parents by using some kind of parent training which was given additionally to child CBT. Such an approach implies that most therapeutic change is still delivered by the therapist and not by the parents using "transfer of control" of CBT skills (Ginsburg, Silverman, \& Kurtines, 1995). Second, the reviewed studies did not involve siblings and are therefore no "true" family treatments. Sibling involvement may affect the whole family structure, and free the anxious child from the position of a "scapegoat". Third, several studies recruited children by means of advertisements. These children and their families might have less severe problems and therefore might not need additional family treatment.

In this randomised controlled trial, family CBT was given based on the transfer of control principles including both parents and siblings of clinically referred youth, aged 8 to 18 , with severe anxiety disorders. It was hypothesised that; (I) CBT is more beneficial than waitlist, (II) family CBT is more effective than child CBT, especially if (III) parents suffer from anxiety disorders themselves, and (IV) young children benefit more from family CBT, whereas older children benefit more from individual CBT.

\section{Method}

\section{Participants}

Participants were 128 children, referred to eight participating Dutch childhood mental health care centres. Families were randomly assigned to individual CBT $(n=64)$ or family CBT $(n=64)$. Twenty-five families were assigned to a natural 8-12 week wait-list. Inclusion criteria were (1) age 8 to 18, (2) primary anxiety disorder (except OCD and PTSD), (3) IQ $\geq 80$, (4) at least one parent willing to participate. Children were excluded if suffering from (1) substance abuse, (2) current suicide attempts, (2) psychoses, (3) autism spectrum disorders, (4) untreated attention deficit hyperactivity disorder, and (5) if they used medication for their anxiety, unless they stopped medication before treatment or dosage was kept constant during treatment and follow-up. 
There were 76 girls (59\%) and 52 boys $(41 \%)$, from 8 to 17 years $(M=$ 12.4, $S D=2.7)$. Most were Caucasian $(n=126,98 \%)$ and $58(45 \%)$ attended primary school. The remaining $70(55 \%)$ attended high schools, of low $16(23 \%)$, average $21(30 \%)$ and high $25(36 \%)$ academic level. In addition, $8(11 \%)$ followed vocational training.

Participants were assessed using a semi-structured interview, the Anxiety Disorder Interview Schedule for DSM-IV, child and parent version (Silverman, \& Nelles, 1988). The mean number of diagnoses per child was 2.9 $(S D=1.7), 104(81 \%)$ had co-morbid anxiety disorders, and the mean severity level of the primary diagnosis was $7.1(S D=1.0)$ (see Table 1).

Table 1 Children's primary and co morbid compound diagnosis based on the ADIS $(n=128)$

\begin{tabular}{lcccc}
\hline & \multicolumn{2}{c}{ Primary diagnosis } & & \multicolumn{2}{c}{ Comorbid diagnosis } \\
\cline { 2 - 3 } & $N$ & $\%$ & & $\%$ \\
\hline Diagnosis & & & & $\%$ \\
Social phobia & 41 & $32 \%$ & 45 & $35 \%$ \\
Separation anxiety disorder & 34 & $27 \%$ & 21 & $16 \%$ \\
Generalised anxiety disorder & 23 & $18 \%$ & 45 & $35 \%$ \\
Specific phobia & 21 & $16 \%$ & 54 & $42 \%$ \\
Panic disorder & 9 & $7 \%$ & 20 & $16 \%$ \\
Dysthymic disorder & & & 23 & $18 \%$ \\
Attention deficit Hyperactivity disorder & & 10 & $8 \%$ \\
Posttraumatic-stress disorder & & & 8 & $6 \%$ \\
Depressive disorder & & 8 & $6 \%$ \\
Obsessive-compulsive disorder & & 6 & $5 \%$ \\
Conduct disorder & & 2 & $2 \%$ \\
Oppositional defiant disorder & & 1 & $1 \%$ \\
\hline
\end{tabular}

Note: Children with OCD and PTSS as primary anxiety disorders were not included in this study

Of the parents, 117 (91\%) fathers and $126(98 \%)$ mothers participated of whom one mother $(1 \%)$ and one father $(1 \%)$ were adoption parents and $3(3 \%)$ fathers were stepfathers. Two mothers (2\%) did not participate due to death (1) and maternal social phobia (1). Eleven fathers did not participate due to; death (5), homelessness (1), language problems (1), drugs use (1) and lack of motivation (3). Maternal and paternal mean age was respectively, $41.8(S D=$ $4.8)$ and $45.0(S D=5.1)$ years. Both biological parents were assessed, using the ADIS adult interview (DiNardo, Brown, \& Barlow, 1994). In 39\% $(n=50)$ of the families, at least one parent had a current anxiety disorder. One family in family CBT was excluded from questionnaire-analysis due to incomplete data.

\section{Design and Procedure}

At intake, families who met the inclusion criteria received and signed informed consent. Research assistants blind to treatment condition conducted the ADISinterviews and questionnaire assessments at pre-treatment, post-treatment and 3-month follow-up. Following the pre-treatment assessment of parents, families 
with and without an anxiety-disordered parent were randomly assigned to family or individual CBT. The active treatment conditions consisted of weekly sessions, which lasted for maximum 90 minutes. During the 3-month follow-up period, the therapist called the families twice, but no other treatment was given. At 3-month follow-up, the ADIS-C and ADIS-P were re-administered and all family members filled in the questionnaires. After the assessment, the therapist informed the family about their results and if necessary informed them about further treatment possibilities. Twenty-five families were assigned to an 8 to 12 week waitlist and were given a reduced battery of questionnaires and the ADIS$\mathrm{C}$ and $\mathrm{P}$ before and after wait-list. Afterwards $76 \%(\mathrm{n}=19)$ were randomly assigned to individual or family CBT and the remaining 6 families received treatment as usual because the trial had finished (see Graph 1).

Graph 1 Flow chart of the randomisation and dropout rates of the included children

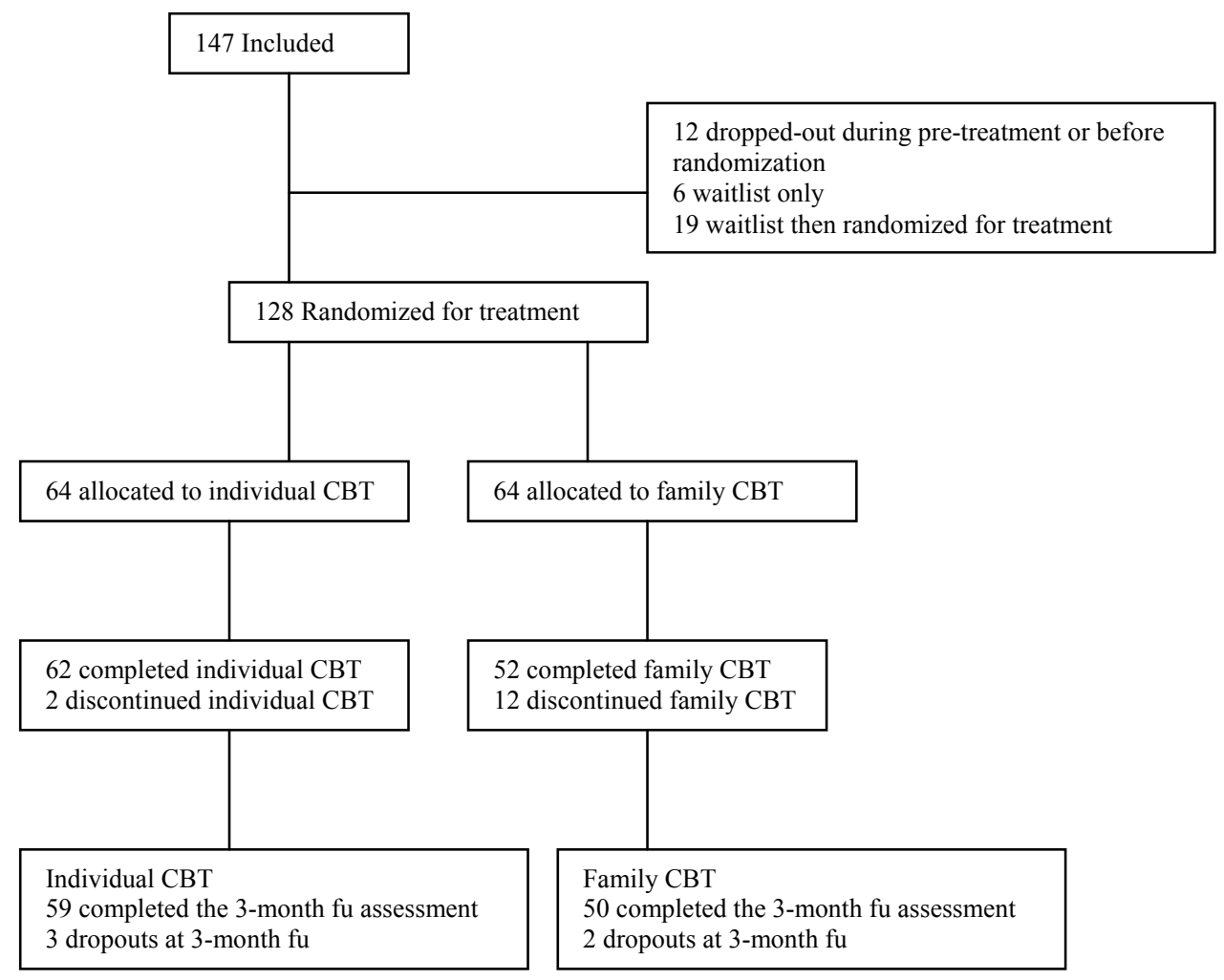


Assessments

\section{Interviews}

Children's diagnostic status was assessed using the ADIS-C and ADIS-P. The ADIS-C/P is a semi-structured diagnostic interview measuring anxiety disorders and other DSM-IV childhood psychopathology. Each diagnosis is rated on severity ranging from 0 to 8,4 indicating a clinical diagnosis. Child and parents ratings are combined in a compound summary. The ADIS-C/P possesses good inter-rater (Silverman, \& Nelles, 1988) and high test-retest reliability (Silverman, \& Eisen, 1992). Parental anxiety diagnoses were obtained by the ADIS adult version (DiNardo et al., 1994). Brown, DiNardo, Lehman, \& Campbell, 2001) which possesses good to excellent reliability.

\section{Questionnaires}

The Screen for Child Anxiety Related Emotional Disorders, child and parent report were administered to assess the child's anxiety symptoms. The validity and reliability of SCARED-71, in which the subscale social phobia is enlarged, is satisfactory (Bodden, Bögels, \& Muris, submitted). Furthermore, the wellestablished State Trait Anxiety Inventory (Spielberger, 1973) child and parent version and the Child Behaviour Checklist (Achenbach, \& Edelbrock, 1983) were administered. Children completed the Children's Automatic Thought Scale (Schniering, \& Rapee, 2002), to measure negative self-statements across both internalizing and externalizing problems. The CATS consists of 4 subscales: physical threat, social threat, personal failure and hostile intent. The CATS possesses good internal consistency and satisfactory test-retest reliability (Schniering, \& Rapee, 2002).

\section{Therapists, research assistants, and treatment/assessment integrity}

Therapists (psychotherapists, behavioural therapists, or health care psychologists) were trained by Lynn Siqueland and Susan Bögels, used a detailed session-to-session protocol, and had weekly group supervision by a local expert. All therapy sessions were audio taped. Afterwards, one audiotape per treatment was randomly selected and reviewed by two trained psychologists to assess treatment integrity. Treatment integrity check indicated perfect agreement on condition (Individual or Family CBT), ICC $=1.00$. A scale was construed to assess achievement of treatment goals per session separately for individual and family CBT. Interrater agreement for obtained therapy goals was .92 for individual CBT and .84 for family CBT. Therapy goals were largely obtained $(M=2.4, \mathrm{SD}=0.7$ for Individual $\mathrm{CBT}$ and $M=2.5, S D=0.6$ for family CBT on a scale of $0=$ not obtained, $1=$ a bit, $2=$ satisfactory, $3=$ good.

Research assistants were trained in the ADIS and rated four gold standard videotapes provided by the Child and Adolescent Anxiety Disorder Clinic of the Temple University in Philadelphia, USA. Their ratings had to match the diagnoses given by the experienced interviewers from Temple. Moreover, each research assistant videotaped 4 of his/her own interviews, which were re-rated by 2 trained students. The mean inter-rater agreement for diagnoses (kappa) was 0.89 for the ADIS-C and 0.83 for the ADIS-P. 


\section{Therapies}

Both therapies consisted of 13 sessions and had its' own therapist manual and patient workbooks. Both therapies began with one introduction session in which family functioning was assessed and principles of CBT were introduced. Individual CBT

Individual CBT was loosely based on Kendall's Coping Cat (Kendall, 1994). The focus was on (1) psycho education and registration of fear, (2) cognitive restructuring and behaviour experiments, (3) exposure in vivo by means of a fear hierarchy and reinforcement and (4) relapse prevention. Parents were involved as little as possible, in order to maximise treatment differences. At the start of treatment they were informed about CBT, at session 4 they were involved in the fear hierarchy and reward system and in the last session the therapy results were shared with the parents.

\section{Family CBT}

Family CBT (Bögels, \& Siqueland, 2006) was developed based on earlier work of Siqueland and Diamond (1998), Ginsburg et al. (1995) and Barrett et al. (1996). Three sessions are with the child alone, 2 with child and parents, 5 sessions with parents alone and 3 sessions involve the whole family, including siblings. The focus of family CBT is threefold; (1) family CBT includes dyadic sessions, in which both child AND parents are taught to reduce their own anxiety using CBT skills. Parents learn to model courageous behaviour and guide and reward the exposure of the child; (2) modifying parental dysfunctional beliefs about parenting, the anxious child, the safety of the child's world and their role as parents and modifying the child's dysfunctional beliefs about the parents and about using anxiety as means of communication; (3) modifying problematic family interaction by improving communication and problem solving. One major focus is to grant autonomy to the child. Parental conflict and communication problems about handling their anxious child are addressed in a session alone with the parents. A pilot study of 17 families showed that $59 \%$ of the children were free of their primary anxiety disorder at 3-month follow-up (Bögels, \& Siqueland, 2006).

\section{Data analysis}

All analyses were done using the intent to treat principle, including the children who started treatment but dropped-out. Missing data were predicted using the missing value analysis of SPSS based on regression models using available data of the group mean, the available data of the individual and the pattern of change of the group. For treatment-dropouts and follow-up-dropouts, last assessments were carried forward, assuming no change. The percentage of children free of all anxiety disorders was used as the primary outcome measure, since the primary diagnosis is less stable (Costello, Mustillo, Erkanli, Keeler, \& Angold, 2003). Mother and father scores were aggregated for SCARED, STAI and CBCL. Effect sizes were calculated by dividing the change score by the standard deviation of change. To measure whether the scores of the anxious 
child fell within the range of the normal population, cut-off scores for SCARED, STAI and CBCL were applied. Binary logistic analysis was used to analyze nominal data (free of anxiety disorders and below cut-off point). Ordinal data (questionnaires) were analysed by ANOVAs using change scores (pre minus post or follow up).

\section{Results}

\section{Pre-treatment comparison}

No differences at pre-treatment occurred across family versus individual CBT and waitlist versus CBT with respect to demographic variables; age, gender, marital status, child and parent's education level and psychopathology (number and severity of diagnoses), all $F(1,126)$ 's $<3.8, p>0.1, x^{2}(1, n=128)=$ $0.6, p>0.1$ for gender and $x^{2}(1, n=128)=3.7, p>0.1$ for parental marital status.

\section{Treatment effectiveness}

After the 3-month waiting period, $100 \%$ of the 25 -waitlisted children still suffered from an anxiety disorder. In contrary, at post-treatment, $52(41 \%)$ children were free of all anxiety disorders, $x^{2}=15.4, p<0.01$, and $71(56 \%)$ free of their primary anxiety diagnosis. At 3-month follow-up, these figures were 66 $(52 \%)$ and $86(67 \%)$, respectively. Only $14(11 \%)$ children did not benefit from treatment, that is, no decline of severity of anxiety diagnoses. Overall, no significant time effect was found on the questionnaires after waitlist, $F$ Hot. (5, 20) $=1.6, p>0.1$.

\section{Family versus individual CBT}

\section{Diagnostic interview}

Binary logistic regression revealed a main effect for condition at post-treatment, corrected for parental anxiety and age, Wald $(1)=9.9, p<0.01, O R=3.7$. Of the 64 children in individual CBT, 53\% no longer met criteria for any anxiety disorder compared to $28 \%$ in family CBT. At 3-month follow-up, these figures were no longer significantly different, respectively $56 \%$ and $47 \%$, Wald $(1)=1.2$, $p>0.1$ (see Table 2). The similar results were found when using the data of families who completed treatment. Of the 62 children who completed individual CBT, 55\% no longer met criteria for any anxiety disorder, whereas $33 \%$ no longer suffered from any anxiety disorder after completing family CBT, Wald $(1)=7.6, p<0.01, O R=3.4$. At 3-month follow-up, the percentages were $58 \%$ for individual CBT and $54 \%$ for family CBT, Wald (1) $=0.3, p>0.1$.

At post-treatment and follow-up, the predicted interaction effect between condition and parental anxiety was not found, all Wald (1) $<1.0, p>$ 0.1 . Hence family CBT was not more efficacious if parents suffered from anxiety disorders. However, a main effect for parental anxiety was found at posttreatment Wald (1) $=10.0, \mathrm{p}<0.01, O R=4.0$, and at 3-month follow-up, Wald (1) $=4.3, p<0.05, O R=2.2$. If neither parent suffered from an anxiety disorder, 40 $(51 \%)$ children at post-treatment and $46(59 \%)$ at follow-up no longer met 
criteria for any anxiety disorder compared to 12 (24\%) and 20 (40\%) children of which one or both parents had anxiety disorders.

The expected interaction effect between age and condition did not occur at post-treatment and follow-up, all Wald (1) $<0.4, p>0.1$. Thus, family CBT was not more effective for young children and individual CBT was not more effective for adolescents. In spite of this, a main effect for age occurred at posttreatment (but not at follow-up), Wald $(1)=7.1, p<0.01, O R=1.2$. Older children benefited less from CBT than younger children as computed using the biserial correlation between age and presence of an anxiety disorder, $R_{\mathrm{b}}=R_{\mathrm{pb}} \sqrt{ }$ proportion anxiety * proportion no anxiety/ $y$ (ordinate) $=.19 \sqrt{0.406}$ * $0.594 / .3876=24$.

Table 2 Percentage anxiety disorder and primary anxiety disorder free children after individual ( $n$ $=64)$ and family CBT $(n=64)$

\begin{tabular}{|c|c|c|c|c|c|c|c|c|}
\hline & \multicolumn{4}{|c|}{ Post treatment } & \multicolumn{4}{|c|}{ 3-month follow-up } \\
\hline & \multicolumn{2}{|c|}{ Individual CBT } & \multicolumn{2}{|c|}{ Family CBT } & \multicolumn{2}{|c|}{ Individual CBT } & \multicolumn{2}{|c|}{ Family CBT } \\
\hline & $\bar{N}$ & $\%$ & $\bar{N}$ & $\overline{\%}$ & $\bar{N}$ & $\%$ & $\bar{N}$ & $\%$ \\
\hline \multicolumn{9}{|l|}{ ADIS } \\
\hline $\begin{array}{l}\text { Free of } \\
\text { anxiety disorder } \\
\text { Free of Primary }\end{array}$ & 34 & $53 \%$ & 18 & $28 \%$ & 36 & $56 \%$ & 30 & $47 \%$ \\
\hline anxiety disorder & 45 & $70 \%$ & 26 & $41 \%$ & 47 & $73 \%$ & 39 & $61 \%$ \\
\hline
\end{tabular}

\section{Questionnaires}

No main effects were found on pre-post change scores for either treatment condition, $F$ Hot. $(6,110)=.05, p>0.1$, child's age, $F$ Hot. $(54,650)=.50, p>0.10$ or parental anxiety, $F$ Hot. $(6,110)=.01, p>0.1$ as measured by the SCARED, STAI, CATS and CBCL internalizing scale. No interaction effects occurred between treatment condition and parental anxiety, $F$ Hot. $(6,86)<0.1, p>0.1$ or child's age, $F$ Hot. $(48,506)<0.6, p>0.1$, indicating no support for the hypotheses that family CBT is more effective if parents suffer from anxiety disorders and that family CBT is more effective in young children. However, a significant interaction of age by parental anxiety was found, $F$ Hot. $(54,506)=$ $1.5, p<0.05$, on the STAI-C, $F(9,91)=2.4, p<0.05$, STAI-P $F(9,91)=2.4, p<$ 0.05 , and CBCL internalizing scale, $F(9,91)=2.2, p<0.05$. A simple inspection of means revealed a pattern indicating that of the parents without anxiety disorders, a higher percentage of younger children (9 to 12) benefited from treatment compared to parents who had anxiety disorders. The older children (13-17) improved irrespective of parental anxiety.

The pre-follow-up MANOVA revealed no significant main effects for condition, parental anxiety, both $F$ Hot. $(6,110)$ 's $<0.9, p>0.1$ or the child's age, $F$ Hot. (54, $650)=.53, p>0.1$. One significant overall interaction was found, treatment condition by parental anxiety, $F$ Hot. $(6,86)=2.2, p<0.05$. Post-hoc ANOVAs showed that all six variables contributed to the interaction. Inspecting the 
Individual versus family CBT

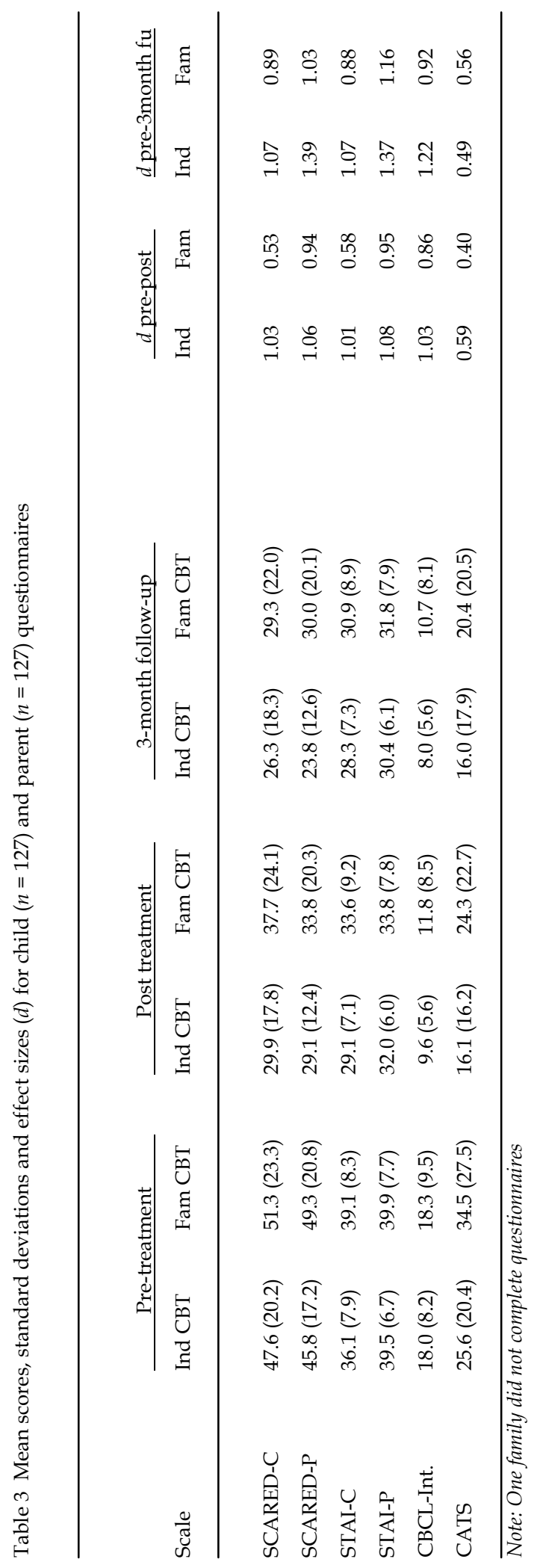


means showed that, in contrast to our hypothesis, if parents do not suffer from anxiety disorders, family CBT was more beneficial, whereas if parents had an anxiety disorder, individual CBT was more effective. Treatment effect sizes (see Table 3) were generally high, increased at follow-up, were higher for individual CBT than family CBT, and higher for parent reports compared to child reports.

\section{Clinical significant change}

Cut-off scores obtained from literature were used to assess the percentage of children falling into the normal range (CATS; Bodden, \& Bögels, submitted, SCARED; Bodden et al., submitted, STAI; Bakker, van Wieringen, van der Ploeg, \& Spielberger, 1989 and CBCL; Verhulst, van der Ende, \& Koot, 1996), see Table 4. No pre-treatment differences on cut-off scores existed, all Wald (1) $<2.9, p>0.1$. A main effect for treatment type was found on post-treatment cutoff scores of STAI-C, corrected for pre-treatment cut-off scores, Wald $(1)=3.9, p$ $<0.05, O R=5.4$, indicating that significantly more children fell into the normal range after individual than after family CBT; $97 \%$ versus $83 \%$. Furthermore, a main effect of parental anxiety on CATS was found, Wald $(1)=5.9, p<0.05$, OR $=2.8$. If parents had no anxiety disorders $74 \%$ of their children fell below the cut-off point of the CATS compared to $48 \%$ if parents suffered from anxiety disorders. Interestingly, an interaction effect of treatment by parental anxiety occurred on SCARED-C, Wald $(1)=3.6, p=0.057, O R=4.6$. Inspection of this interaction showed that in contrast to the hypothesis, if parents suffered from anxiety disorders, more children fell into the normal range with regard to anxiety symptoms after individual CBT (46\%) compared to family CBT (19\%). If parents did not suffer from an anxiety disorder, the percentages were $55 \%$ for individual CBT and $62 \%$ for family CBT. Neither a main effect for child's age nor an interaction effect for child's age by condition was found.

Table 4 Percentages of children underneath the cut-off point of the questionnaires after individual and family CBT

\begin{tabular}{|c|c|c|c|c|c|c|c|c|}
\hline & \multicolumn{4}{|c|}{ Post treatment } & \multicolumn{4}{|c|}{ 3-month follow-up } \\
\hline & \multicolumn{2}{|c|}{ Ind CBT } & \multicolumn{2}{|c|}{ Fam CBT } & \multicolumn{2}{|c|}{ Ind $\mathrm{CBT}$} & \multicolumn{2}{|c|}{ Fam CBT } \\
\hline & $N$ & $\%$ & $N$ & $\%$ & $N$ & $\%$ & $N$ & $\%$ \\
\hline \multicolumn{9}{|l|}{ Scale } \\
\hline \multicolumn{9}{|l|}{ SCARED } \\
\hline Child & 33 & $52 \%$ & 28 & $44 \%$ & 43 & $67 \%$ & 39 & $62 \%$ \\
\hline Parent & 19 & $30 \%$ & 23 & $37 \%$ & 30 & $47 \%$ & 24 & $38 \%$ \\
\hline \multicolumn{9}{|l|}{ STAI } \\
\hline Child & 62 & $97 \%$ & 53 & $83 \%$ & 62 & $97 \%$ & 58 & $91 \%$ \\
\hline Parent & 62 & $97 \%$ & 57 & $89 \%$ & 63 & $98 \%$ & 61 & $95 \%$ \\
\hline CBCL-Int. & 56 & $88 \%$ & 50 & $78 \%$ & 58 & $91 \%$ & 48 & $75 \%$ \\
\hline CATS & 47 & $73 \%$ & 34 & $54 \%$ & 46 & $72 \%$ & 39 & $62 \%$ \\
\hline
\end{tabular}


Using follow-up cut-off scores, a main effect for treatment condition was found on CBCL internalizing scale, Wald $(1)=7.4, p<0.01, O R=4.8$, showing a beneficial effect for individual (91\%) compared to family (75\%) CBT. The interaction effect of treatment by parental anxiety remained on SCARED-C, Wald $(1)=4.7, p<0.05, O R=5.5$, showing beneficial effects for individual CBT (75\%) compared to individual CBT $(46 \%)$ if parents had anxiety disorders. If parents did not suffer from an anxiety disorder, the percentages were $63 \%$ for individual CBT and $73 \%$ for family CBT. Again, no main effect of child's age or interaction effect of child's age by condition occurred.

\section{Discussion}

The present study investigated the effects of family CBT compared to individual $\mathrm{CBT}$ in clinically anxious children. The main results can be summarised as: (I) CBT was more effective than wait-list; (II) individual CBT was more effective than family CBT at post-treatment; (III) at follow-up, family CBT and individual CBT were equally effective; (IV) both treatments were less effective if parents suffered from anxiety disorders themselves both at posttreatment and follow-up; and $(\mathrm{V})$ both treatments were more effective in younger children at post-treatment.

Contrary to hypothesis, we found no added effect of family CBT. Unexpectedly, the reverse was found, namely individual CBT was more beneficial at post-treatment. The finding that family involvement does not add to the effectiveness of individual CBT is consistent with the majority of studies evaluating this question (In-Albon, \& Schnieder, in press), but this is the first study showing individual CBT to be more effective. Several explanations can be given. First, individual CBT might be more autonomy encouraging to the anxious child than family CBT because the child receives therapy and the possible success alone. Second, the number of family therapy sessions might have been insufficient to address all the topics thoroughly: parental anxiety, family functioning and communication, as well as the child's anxiety. These topics may need more time to be fully covered. Third, since family CBT works by means of transfer of control, most information and guidelines are given through the parents. As such, distortions can occur in the transfer of information from parent to child. Since therapists are thoroughly trained in CBT principles they may be more capable of coaching children. Note that this was the first RCT in which almost no individual CBT was given to the child in the family condition. Fourth, it may have been difficult to fully engage and motivate parents and siblings in family CBT because they were not the ones referred to treatment. Fifth, parents might be more defensive in family CBT since they might feel blamed for the child's anxiety.

At 3-month follow-up, family CBT and individual CBT were equally effective, mainly because family CBT "catches up". This can be explained by the process of Sowing and Reaping (Nauta et al., 2003). During family treatment the 
parents learned training skills (sowing), which can be used to master the child's anxiety after treatment (reaping).

With respect to overall effectiveness, both treatments appeared highly efficacious (individual CBT 56\% and family CBT 47\% free of all anxiety disorders at 3-month follow-up) and compare well with the overall remission rate of $57 \%$ from a meta-analysis of CBT for childhood anxiety disorders (Cartwright-Hatton et al., 2004). Note that some studies included in this metaanalysis recruited children predominantly by means of advertisements, who are less severe anxious and therefore may easier meet the stringent criterion of free of all anxiety disorders (Weisz, Weiss, Han, Granger, \& Morton, 1995), and did not include dropouts. In addition, ratings of severity of the anxiety disorders in our sample indicated that only 14 children (11\%) did not improve. In addition, the average effect size was rather high, 1.2 for individual CBT and 1.1 for family CBT. In comparison, In-Albon and Schneider (in press) found an overall effect size of .84 for CBT, suggesting that even the less effective therapy in this study, family CBT, was highly efficacious.

In contrast to our hypothesis and to Cobham et al. (1998), family CBT was not more effective if parents suffered from anxiety disorders. In contrast, questionnaire results showed family CBT to be more effective if parents had no anxiety disorders. A more general finding was that CBT was less effective if parents suffer from anxiety disorders. It has been proposed that parental psychopathology may hinder the process of transfer of control of CBT skills from parents to child (Ginsburg et al., 1995). Parents with current anxiety disorders might have been too anxious to transmit CBT to their child. Since $20 \%$ of the anxiety disordered mothers suffered from co-morbid depression, maternal depression might have also contributed to the less beneficial child outcomes, in line with previous research (Southam-Gerow, Kendall, \& Weersing, 2001). Research into the effects of parental treatment of anxiety disorders on child outcomes might shed more light on this issue.

Also, in contrast to our hypothesis and Barrett et al. (1996), we did not find that younger children benefited more from family CBT and older children more from individual CBT. However, child age influenced treatment outcome in general. Consistent with Southam-Gerow et al. (2001) and Hudson et al. (2002), in our study CBT was less effective in older children (13 years and older). Several explanations for the less beneficial outcomes for adolescents in our study can be given. First, the same therapy workbooks were applied for children and adolescents and therefore may not have been developmentally sensitive. Some exercises may have been too childish and as a consequence adolescents might not have fully engaged in therapy, reducing efficacy. Second, adolescents function more independent and are less cooperative so therapeutic alliance is more difficult to establish (Hudson et al., 2002). Third, and most likely, adolescents may have established more persistent negative cognitive and behavioural patterns (e.g. avoidance) due to the longer duration of anxiety 
symptoms in comparison with younger children. Therefore, it might be more difficult to change these patterns.

Interestingly, $86 \%$ of the treatment-dropouts were in the family CBT condition. Inspection of reasons for dropout in family CBT suggests that in some cases it was hard to motivate family members, since the child's anxiety was seen as the primary focus of treatment. Post-hoc analyses of pre-treatment differences between family CBT completers versus dropouts were carried out to further investigate reasons for family treatment-dropout. No differences were found regarding the following child characteristics; age, gender, number and severeness of anxiety disorder. Families did not differ on parental anxiety disorder, parental psychopathology, number of children, and social economic status. However, the percentage of broken homes was higher (36\%) in family CBT dropouts than in family CBT completers $(14 \%), \chi^{2}(1)=3.2, p=0.08$. Furthermore, the percentage of housewives was significantly higher in the dropout-group $(73 \%)$ compared to the completer group $(28 \%), \chi^{2}(1)=8.2, p<$ 0.05 . Hence, more complexity in families and less employment of mothers occurred to be associated with family treatment-dropout. A highly speculative explanation is that being a housewife is associated with more overprotection of the anxious child and more resistance to change. Clearly, more research is necessary to understand treatment-dropout during family treatment.

This study has several limitations. First, although CBT was more effective than waitlist, this might not provide enough evidence for its' efficacy. Two studies indicate that CBT is not superior to educational support (Last, Hansen, \& Franco, 1998; Silverman et al., 1999). Second, families were randomly assigned to family CBT. Perhaps family CBT will be more beneficial if given based on either family preference or impairment in family functioning.

\section{Clinical Implications}

This study has shown that individual CBT is more effective than family CBT, at least in the short term. Taking into consideration the majority of literature, indicating that parental involvement does not add to the effects of child CBT, one can question whether involving parents in therapy is needed. Family CBT is less feasible and is also expected to be more costly due to invested treatment time of family members. Some of this study's results suggest that the clinical intuition to involve parents especially if they are anxious may not be best practice. For "healthy" parents family CBT does seem an alternative to childfocused CBT. 


\section{Chapter}

\section{Costs and cost-effectiveness of family CBT versus individual CBT in clinically anxious children}

Denise H.M. Bodden, Carmen D. Dirksen, Susan M. Bögels, Carla Appelboom, Karen C.M.M.J. Appelboom-Geerts, Andries G. Brinkman, Else de Haan, Jaap Ringrose, \& Maaike H. Nauta 


\begin{abstract}
Objective: To investigate the cost-effectiveness of family Cognitive Behaviour Therapy (CBT) compared with individual CBT.

Design: Clinically anxious children referred to treatment were randomly assigned to family or individual CBT and were assessed at pre-treatment, post treatment, 3-months and 1-year after treatment.

Outcome measures: Cost-effectiveness ratios were calculated expressing the incremental costs per anxiety-free child and the incremental costs per Quality Adjusted Life Year (QALY) for the referred child.

Results: Both societal costs and effectiveness were not significantly different between individual and family CBT. However, the point estimates of the costeffectiveness ratios resulted in dominance for individual CBT, indicating that individual CBT is more effective and less costly compared to family CBT. These results were confirmed by bootstrap analyses and cost-effectiveness acceptability curves. Several secondary and sensitivity analyses showed that results were robust.

Conclusion: It can be concluded that family CBT is not a cost-effective treatment, compared to individual CBT.
\end{abstract}




\section{Introduction}

Mental illnesses cause a high burden on society. Of all mental illnesses, the total costs are highest for anxiety disorders, namely 46.6 billion dollars in the USA, accounting for $31.5 \%$ of total expenditures for mental health in 1990 (Dupont et al., 1996). In the Netherlands, the costs of anxiety were 180 million euro in 1999, accounting for $0.5 \%$ of the health care budget. More specifically, health care costs due to anxiety disorders in children and young adults aged 1 to 24 amounted to 21.6 million euros (Polder, Takken, Meerding, Kommer, \& Stokx, 2002).

Anxiety disorders affect 5 to $15 \%$ of children and adolescents (Bernstein, Borchardt, \& Perwien, 1996). In the Dutch population, a six-month prevalence rate of $23.5 \%$ was found among 13 - to 18 -year-olds, when impairment in daily functioning was taken into account the rate dropped to 9.7\% (Verhulst, van der Ende, Ferdinand, \& Kasius, 1997). Anxiety disorders substantially influence quality of life of the child and are a burden for the family and society, resulting in high costs (Leon, Portera, \& Weissman, 1995).

Research on the effectiveness of treatments of childhood anxiety disorders focuses mostly on cognitive behavioural therapy (CBT) and demonstrates that CBT is efficacious (Cartwright-Hatton, Roberts, Chitsabesan, Fothergill, \& Harrington, 2004; In-Albon \& Schneider, in press). Evidence for the added value of parental involvement in CBT is inconsistent. For instance, Barrett, Dadds, and Rapee (1996) found that individual CBT with additional family management was more effective than CBT alone. In contrast, a recent study of Nauta, Scholing, Emmelkamp, and Minderaa (2003) and a recent metaanalysis (In-Albon \& Schneider, in press) did not show a superior effect of parental involvement. Recently, a new family based CBT was developed, which includes both parents and siblings of anxious children (Bögels \& Siqueland, 2006). In this family CBT, child and parents are taught to reduce their own anxiety using CBT skills. Furthermore, parents learn to model, guide and reward the exposure of the child and dysfunctional thoughts and problematic family interaction are being modified.

Several authors (Fals-Stewart, Yates, \& Klostermann, 2005; Steinglass, 1996) have argued the need for economic evaluations of family treatment because of their potential cost advantages. Due to the multiplier effect, the positive effects of treating a family member may also transfer to other family members. Besides, by providing a more stable family environment, the chance that positive treatment effects will be undone when the child returns to the family will be reduced.

A cost-effectiveness analysis is a method to evaluate and compare outcomes and costs of interventions (Drummond, O'Brien, Stoddart, \& Torrance, 1997; Gold, Siegel, Russell, \& Weinstein, 1996) with the aim to improve resource allocation decisions by policy makers and insurers, and consequently, to improve individual, societal and economical wellbeing 
(Knapp, 1997). To date, no studies have addressed the costs associated with CBT in children with anxiety disorders and subsequently, its cost-effectiveness.

Offering a cost-effective treatment to children with anxiety disorders is expected to reduce the costs associated with the consequences of anxiety disorders. On the short term it is expected that the child and family's health, quality of life and parental time released will improve. Society as a whole may benefit from better social functioning, less usage of services and increased productivity of the parents. On the long term, effective interventions may prevent self-induced illness, improve attainment of education, and improve social functioning. The expected benefits for society in the long run concern less crime rates, less alcohol and drug abuse, less social care usage, better earning potential for the child, less unemployment of the child as an adult, and less health care usage (Greenberg et al., 1999; Knapp, 1997; Leon et al, 1995; Marciniak, Lage, Landbloom, Dunayevich, \& Bowman, 2004; Tudor Edwards \& Thalanany, 2001). All together, the ultimate savings that can be achieved by investing in a cost-effective treatment for children with anxiety disorders may be substantial.

The aim of this study was to compare family CBT to individual CBT with respect to the costs, effects and cost-effectiveness. The incremental costeffectiveness ratios were expressed as the cost per anxiety-free child and the cost per Quality Adjusted Life Year of the referred child, with a time horizon of 15 months.

\section{Method}

\section{Study design}

A multi-centre randomized clinical trial was performed comparing family CBT with individual CBT (Bodden et al., submitted). Inclusion criteria were (1) age 8 to 18 , (2) primary anxiety disorder (except for OCD and PTSD), (3) IQ $\geq 80$, and (4) at least one parent willing to participate. Children were excluded if suffering from (1) substance abuse, (2) current suicide attempts, (2) psychoses, (3) autism spectrum disorders, (4) untreated attention deficit hyperactivity disorder. Children were also excluded if they used medication for anxiety, unless they stopped medication or dosage was kept constant during treatment and followup. After checking the inclusion and exclusion criteria, the child and parents received and signed an informed consent.

After baseline assessment (pre-treatment), which consisted of the Anxiety Disorder Interview Schedule parent-, adult- and child-version to obtain child and parental psychopathology, and a battery of questionnaires including the EuroQol, families were randomly assigned to 12-sessions individual CBT or family CBT. Directly after treatment (post-treatment), 3-months (follow-up 1) and 12-months (follow-up 2) after treatment, research assistants who were unaware of the treatment condition conducted the follow-up measurements 
which consisted of the ADIS parent- and child-version and several questionnaires.

\section{Cost-analysis}

The cost analysis was, according to Dutch guidelines (Oostenbrink, Koopmanschap, \& Rutten, 2005), performed from a societal perspective and included direct health care costs, direct non-health care costs, indirect costs and out-of-pocket costs. Costs were calculated by multiplying the real resources used by the cost price of each resource used. This approach is also known as 'micro-costing' which is a detailed inventory and measurement of resources consumed (Jacobs \& Roos, 1990). Costs were collected and calculated at 2003 euros $\left(1 €=0,087 \$\right.$, July $\left.30^{\text {th }} 2003\right)$ and were discounted at $4 \%$ after 12 months following randomization (Oostenbrink et al., 2005).

Resources used were determined by means of cost diaries (Drummond et al., 1997; Freer, 1980; Goossens, Rutten-van Mölken, Vlaeyen, \& van der Linden, 2000), which were completed prospectively for five times by one of the parents of the anxious child over a period of 2 weeks each; at pre-treatment (2 weeks before treatment), during treatment, at post-treatment, at 3-month follow-up and 1-year follow-up. Each cost diary was presented in a booklet form with instructions and an example diary. Parents recorded the resources used (see Table 3) and the name of the family member for whom the resource use was applicable (child, sibling, mother or father), as well as the reason for the resource use. For analysis, the reported reasons were grouped into (1) due to anxiety, (2) due to psychological problems, (3) due to physical problems, and (4) due to other reasons. For the main cost-effectiveness analysis, only resource use related to anxiety of the child was considered.

Almost all cost prices were obtained by using published Dutch guideline prices (Oostenbrink et al., 2005). Medication prices were obtained from the Medication and aid Information Project database on the Internet (Board of health insurances, 2005) and were based on the Daily Defined Dosage (DDD), which indicates the mean medication usage per adult a day with claw back and Value Added Tax (20\%). An average cost price was calculated for anxiety medication and medication for psychological problems, used in the secondary analysis. As actual cost prices for day treatment were not available, the cost price was derived from the average of actual reimbursements (Board Tariff rates Health care and Care authority, 2005). The cost price of day care was estimated by using a shadow price based on the actual day care costs for children under the age of 4 (Babyfun, 2005). To determine the costs associated with absence from school, actual annual tuition (National Institute of Budget information, 2005) was divided by the total annual hours at school, resulting in a cost price per hour of school absence. Productivity costs of the parents were calculated by means of the friction cost method, based on a mean added value of the Dutch working population (Oostenbrink et al., 2005). The friction costs method only takes into account production losses confined to the period 
needed (usually 90 days) to replace a sick employee. All cost prices per unit of resource use are presented in Table 3.

\section{Effectiveness}

ADIS

Children's diagnosis was assessed using a semi-structured interview the ADIS, which is specifically designed to measure anxiety disorders and other psychopathology in children and adolescents (Silverman \& Nelles, 1988). Each diagnosis is rated on severity ranging from 0 to 8,4 indicating a clinical diagnosis. The scores of both child (ADIS-c) and parents (ADIS-p) on child anxiety were combined in a compound summary. Siblings' anxiety diagnoses were obtained in the same way. The ADIS-c/p has a good inter-rater (Silverman \& Nelles, 1988) and test-retest reliability (Silverman \& Eisen, 1992). Parental anxiety diagnoses were obtained by the ADIS adult-version (DiNardo, Brown, \& Barlow, 1994), which has a good to excellent inter-rater reliability on the majority of diagnoses (Brown, DiNardo, Lehman, \& Campbell, 2001).

$$
\text { EuroQoL (EQ-5D) }
$$

The EQ-5D is a generic health-related quality of life instrument (EuroQolgroup, 1990). The EQ-5D contains 5 dimensions of health-related quality of life, namely mobility, self-care, daily activities, pain/discomfort and depression/anxiety. Each dimension can be rated at three levels: no problems, some problems and major problems. The 5 dimensions can be summed into a health state. Utility values can be calculated for these health states, using preferences elicited from a general population, the so-called Dolan algorithm (Dolan, 1997). The utility values derived from the Dolan algorithm were used to compute Quality Adjusted Life Years (QALYs). The maximum number of QALYs that can be reached with a time horizon of 15 months is 1.25 . Furthermore, the EQ-5D consists of a visual analogue scale (VAS) ranging from zero (worst imaginable health state) to 100 (best imaginable health state). The reliability and validity of the EQ-5D has been established (Brooks with the EuroQol group, 1996). The EQ-5D used in this study, was filled in by the child, mother and father, concerning the child's quality of life. Mother and father scores were averaged into one proxy score because they correlated highly. The proxy score was used for the cost per QALY analysis. The proxy version of the EQ-5D has good construct validity and convergent validity (Stolk, Busschbach, \& Vogels, 2000).

\section{Cost-effectiveness analysis}

In cost-effectiveness analysis, the difference in costs is related to the difference in effectiveness, resulting in an Incremental Cost-Effectiveness Ratio (ICER). In this study effectiveness was expressed as (1) the proportion of anxiety-free children and based on (2) QALYs. The ICERs expressed (1) the incremental costs per anxiety-free child and (2) the incremental costs per QALY. The time horizon of the cost-effectiveness analysis was 15 months. 


\section{Statistical analysis}

Data were analyzed according to the intention-to-treat principle. Missing items in the cost diaries were handled with the Missing Value Analysis of SPSS based on regression models using available data of the group mean, of the individual and the pattern of change of the group. During treatment 8 families in family CBT and 1 family in individual CBT dropped-out, of which respectively 4 and 1 refused further measurements. Another 4 families in individual CBT and 1 in family CBT refused the measurements at follow-up. Costs and effectiveness data of these families were estimated using the last-observation-carriedforward-method (LOCF-method). Cost data were interpolated to a period of 15 months under the assumption that data obtained with the cost diaries were representative for the periods in between.

To investigate whether data were normally distributed a KolmogorovSmirnov test was performed. If cost and quality of life data were not normally distributed, the non-parametric Mann Whitney $U$ test was used to test for significant differences. Binary logistic regression was used to assess differences in the percentage of anxiety free children.

In order to get insight in the uncertainty surrounding subtotal and total costs and due to highly skewed cost distributions, bootstrap simulations were conducted. The bootstrap method estimates the sampling distribution of a statistic through a large number of simulations, based on sampling with replacement (Briggs, Wonderling, \& Mooney, 1997). The results based on 1000 bootstrap replications of the costs for family CBT and individual CBT were used to calculate $95 \%$ confidence intervals $(95 \% \mathrm{CI})$ around the cost-differences, based on the $2.5^{\text {th }}$ and $97.5^{\text {th }}$ percentiles. Bootstrap simulations were also conducted in order to quantify the uncertainty around the ICER (Efron \& Tibshirani, 1993), yielding information about the joint distribution of cost and effect differences. The bootstrapped cost-effectiveness ratios were subsequently plotted in a cost-effectiveness plane, in which the vertical line reflects the difference in costs and the horizontal line reflects the difference in effectiveness. The choice of treatment depends on the maximum amount of money that society is prepared to pay for a gain in effectiveness, which is called the ceiling ratio. Therefore, the bootstrapped ICERs were depicted in a cost-effectiveness acceptability curve showing the probability that family CBT is cost-effective using a range of ceiling ratios. Finally, several secondary analyses were conducted to investigate the robustness of the results for changing several parameters.

\section{Results}

\section{Participants}

In total 128 children participated in the RCT. At pre-treatment, two children from the individual CBT group received clinical treatment and were institutionalized. Since the costs associated with clinical treatment were 
extremely high, persisted over time and were not attributable to treatment condition, these 2 children were excluded from the cost-effectiveness analysis. Furthermore, 3 (2\%) families in individual CBT and $7(6 \%)$ families in family CBT did not fill in the pre-treatment cost diary. Since this information is necessary for interpreting follow-up cost data, these families were excluded from the analysis. Therefore, results with respect to the costs and costeffectiveness analysis are presented for 116 children, of which 59 were randomized to individual CBT and 57 to family CBT.

The children consisted of 72 girls (62\%) and 44 boys (38\%), from 8 to 17 years $(M=12.3, S D=2.6)$. Most were Caucasian $(n=115,99 \%)$ and $53(46 \%)$ attended primary school. The remaining $63(54 \%)$ attended secondary education, of average $(31,49 \%)$ and high $(24,38 \%)$ and specialized $(8,12 \%)$ educational level. The mean number of diagnosis per child was $2.9(S D=1.6)$, whereas the mean severity level of the primary diagnosis was $7.1(S D=1.0)$ (see Table 1).

Table 1 Primary and co morbid compound diagnosis (pre-treatment) based on the ADIS ( $n=116)$

\begin{tabular}{|c|c|c|c|c|}
\hline & \multicolumn{2}{|c|}{ Primary diagnosis } & \multicolumn{2}{|c|}{ Comorbid diagnosis } \\
\hline & $\bar{N}$ & $\%$ & $\bar{N}$ & $\%$ \\
\hline \multicolumn{5}{|l|}{$\overline{\text { Diagnosis }}$} \\
\hline Social phobia & 36 & $31 \%$ & 42 & $36 \%$ \\
\hline Separation anxiety disorder & 32 & $28 \%$ & 20 & $17 \%$ \\
\hline Specific phobia & 20 & $17 \%$ & 47 & $41 \%$ \\
\hline Generalized anxiety disorder & 20 & $17 \%$ & 41 & $35 \%$ \\
\hline Panic disorder & 8 & $7 \%$ & 18 & $16 \%$ \\
\hline Obsessive-compulsive disorder & & & 5 & $4 \%$ \\
\hline Posttraumatic-stress disorder & & & 5 & $4 \%$ \\
\hline Dysthymic disorder & & & 19 & $16 \%$ \\
\hline Depressive disorder & & & 6 & $5 \%$ \\
\hline Attention deficit Hyperactivity disorder & & & 9 & $8 \%$ \\
\hline Conduct disorder & & & 2 & $2 \%$ \\
\hline Oppositional defiant disorder & & & 1 & $1 \%$ \\
\hline
\end{tabular}

Of the parents, 110 (95\%) fathers and 115 (99\%) mothers participated. Maternal and paternal mean age was respectively, $41.8(S D=4.9)$ and $44.9(S D$ $=5.1$ ) years. Both parents were diagnosed, using the ADIS adult interview (26). In $41 \%(n=47)$ of the families, at least one parent had a current anxiety disorder.

Demographic and socio-economic characteristics between individual CBT and family CBT were highly comparable. No statistically significant differences were found regarding the following child characteristics; age, educational level (both $F<3.0, p>0.05$ ) and gender, $x^{2}(1, n=116)=.83, p>0.05$. Also no differences were found on the following parental characteristics; age, educational level, job level, family income, hours of work, hours of volunteer 
work, hours of house hold activities, and hours of leisure time, all $F<3.6$ and $p>$ 0.05 .

\section{Cost-analysis}

At pre-treatment, costs in both groups were not normally distributed (Kolmogorov-Smirnov $Z>2.2, p<0.00$ ). Most families reported no or few costs associated with anxiety of the child. Total societal costs were slightly, but not significantly, higher for individual CBT compared to family CBT (see Table 2). A Mann-Whitney test showed no significant differences between the two groups at pre-treatment on subtotal and total societal costs (all $U>1673, p>.1$ ). The bootstrap analysis also showed no significant differences between the two conditions (see Table 2).

Table 2 Baseline subtotal costs and total societal costs for individual CBT $(n=59)$ and family CBT ( $n$ $=57)$

\begin{tabular}{lccl}
\hline & Ind CBT costs & Fam CBT costs & Incremental costs (95\% CI) \\
\hline Cost category & & & \\
Direct health care costs & $17.02(125)$ & $34.65(178)$ & 17.63 \\
Bootstrapped & $16.67(16.0)$ & $33.35(23.4)$ & $16.68(-32-+69)$ \\
Direct non-health care costs & $4.13(26.7)$ & $0.89(4.7)$ & -3.24 \\
Bootstrapped & $4.12(3.4)$ & $0.91(0.6)$ & $-3.21(-11-+2)$ \\
Indirect costs & $54.82(249)$ & $37.18(132)$ & -17.64 \\
Bootstrapped & $55.52(30.8)$ & $35.82(17.1)$ & $-19.70(-93-+39)$ \\
Out-of-pocket costs & $7.86(54.1)$ & $0.09(0.5)$ & -7.77 \\
Bootstrapped & $7.77(7.1)$ & $0.09(0.1)$ & $-7.68(-23-0)$ \\
Total societal costs & $\mathbf{8 3 . 8 3 ( 2 8 0 )}$ & $\mathbf{7 2 . 8 0 ( 2 6 3 )}$ & $\mathbf{- 1 1 . 0 2}$ \\
Bootstrapped & $84.21(35.9)$ & $73.79(35.8)$ & $-10.42(-105-+93)$ \\
\hline
\end{tabular}

Note: Ind CBT = Individual CBT; Fam CBT = Family CBT

Although not statistically significant, direct health care costs at pretreatment were higher for family CBT. This can be explained by the fact that, out of 3 children in day treatment at pre-treatment, 2 were randomized for family CBT. With respect to the indirect costs, the most prominent costdifferences were present for absence from paid work, absence from school and loss of leisure time, although these differences were not significant. Three parents in individual CBT reported productivity loss, compared to 2 parents in family CBT. At pre-treatment, costs due to absence from school were higher in family CBT due to 6 children reporting relatively more hours of school absence $(M$ hours $=44.5)$, compared to 8 children in individual CBT $(M$ hours $=25.9)$. Loss of leisure time was slightly more reported in family CBT.

Results of the cost analysis over 15 months are summarized in Table 3. No statistically significant differences on subtotal and total societal costs per anxious child were found, Mann-Whitney $U>1485, p>.26$. The same finding was revealed using bootstrapped results. The mean total societal costs 
amounted to $€ 2751.39$ ( $S D=4774)$ for individual $C B T$ and $€ 3051(S D=4582)$ for family CBT. This resulted in an incremental cost-difference of $€ 299$, meaning that family CBT, although not significant, is more costly than individual CBT. Comparable to the pre-treatment situation, costs due to day treatment, leisure time and school absence were higher in family CBT and costs due to absence from paid work were higher in individual CBT. Graph 1 displays the mean total societal costs per cost diary without interpolation. Compared to the pretreatment costs, costs increased during treatment and dropped immediately after treatment, ultimately resulting in lower costs compared to pre-treatment. Moreover, the graph shows that the cost patterns were highly comparable for individual and family CBT.

Graph 1 Mean total societal costs for individual CBT and family CBT per cost diary of 2 weeks

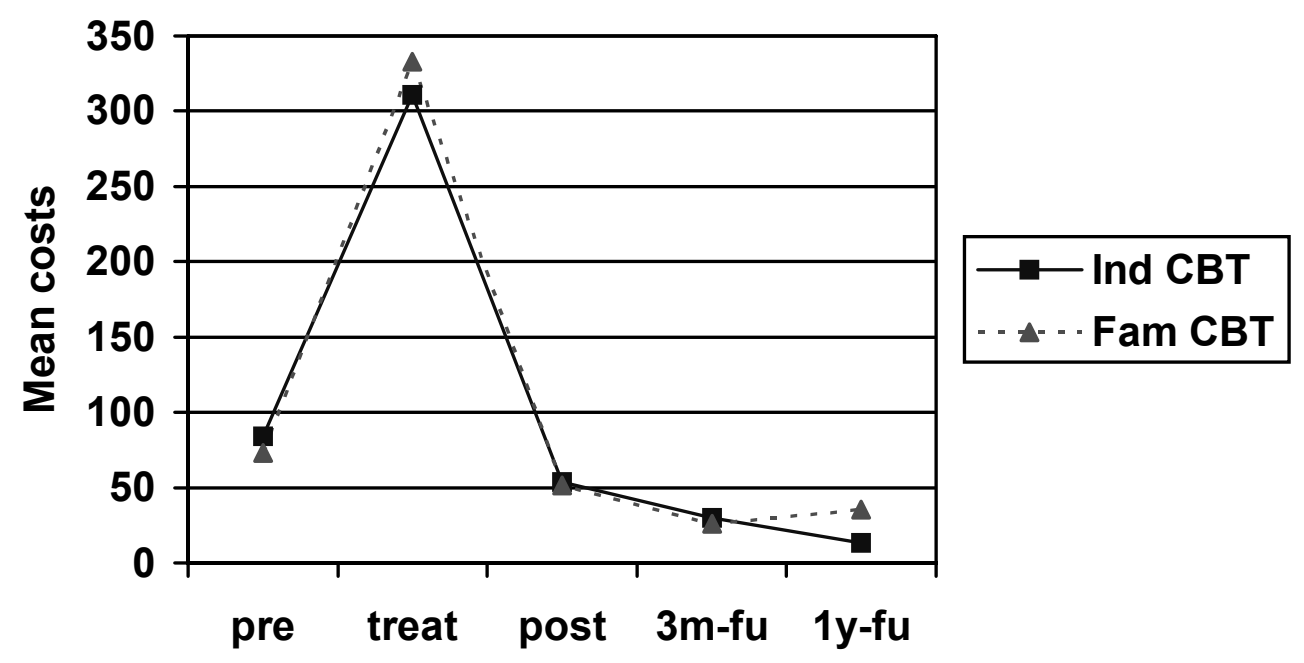

Note: Ind CBT = Individual CBT; Fam CBT = Family CBT

\section{Effectiveness}

ADIS

Directly after treatment, the proportion of anxiety free children was 0.54 for individual CBT and 0.28 for family CBT. At 3-month follow-up, these figures were 0.58 and 0.47 respectively. The proportions of anxiety-free children at 1year follow-up were 0.68 and 0.53 for respectively individual CBT and family CBT, which was not significantly different, Wald $(1)=2.8, p=0.1, O R=0.5$. 
Chapter 7

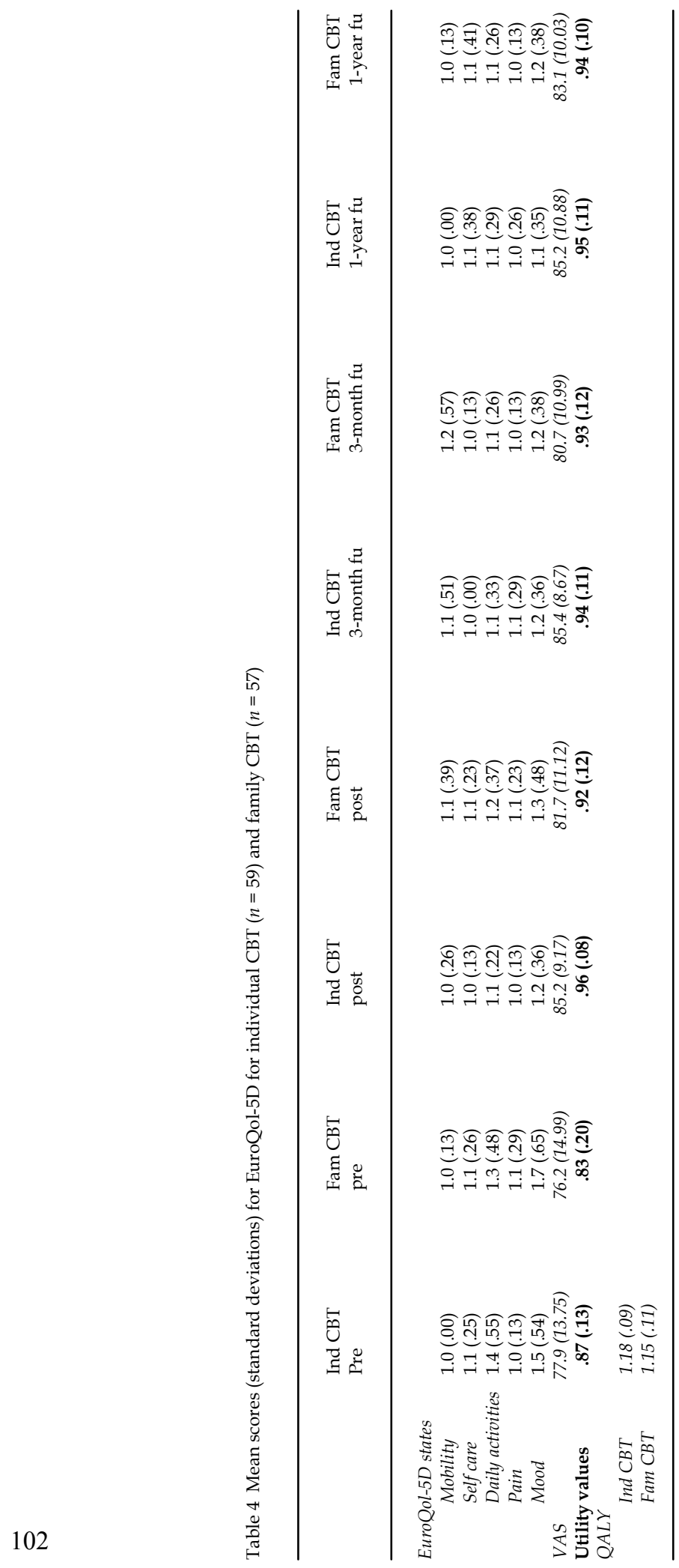


$E Q-5 D$

Results of the EQ-5D at pre-treatment are presented in Table 4. In general, children with anxiety disorders had no problems with mobility, self-care, and pain. The scores on the 'daily activities' and 'mood' dimensions were slightly higher compared to the other dimensions. Although both the VAS value and the utility value were slightly higher for individual CBT compared to family CBT, results were not statistically significantly different between individual CBT and family CBT at pre-treatment (all Mann Whitney $U>1486, p>.09$ ).

In general, the scores on the 'mobility', 'self-care' and 'pain' dimensions remained fairly stable over time, whereas the scores on the 'daily activities' and 'mood' dimensions decreased (see Table 4), resulting in increasing utility values over time. Comparable to the pre-treatment situation, utility values and VAS values at post-treatment, 3-month follow-up and 1-year follow-up were slightly higher in the individual CBT condition compared to family CBT. The number of QALYs was 1.18 for individual and 1.15 for family CBT (Mann Whitney $U=$ $1526, p>.10)$.

\section{Cost-effectiveness analysis}

Costs per anxiety-free child

The ICER based on the costs per anxiety-free child indicated inferiority for family CBT, as total societal costs for family CBT are €299 higher and effectiveness is 0.15 lower (see Table 5). The bootstrapped cost-effectiveness ratios were plotted in a cost-effectiveness plane (Graph 2a), showing that $60 \%$ of cost-effectiveness ratios are situated in the northwest (inferior) quadrant. This indicates that there is a $60 \%$ probability that family CBT is both more costly and less effective compared to individual CBT. Thirty-five percent of the ICERs were located in the southwest quadrant, indicating that family CBT is less costly but also less effective. The northeast quadrant contained $4 \%$ of the ratios, showing that family CBT is more costly and more effective. The remaining $2 \%$ of the ratios were in the southeast quadrant indicating lower costs and higher effectiveness for family CBT, resulting in dominance for family CBT.

Graph $2 \mathrm{~b}$ shows the cost-effectiveness acceptability curve for the percentage of anxiety-free children. For example, using a ceiling ratio of $€ 2500$ per anxiety-free child, the probability that family CBT is cost-effective is $24 \%$. The probability that family CBT is cost-effective decreases from $24 \%$ to $4 \%$ with increasing the ceiling ratio from $€ 2500$ to $€ 37500$.

\section{Cost per QALY}

The ICER based on the cost per QALY also indicated inferiority for family CBT, as total societal costs were $€ 299.13$ higher and the number of QALYs was 0.02 lower for family CBT (see Table 5). Graph 3a shows the bootstrapped costeffectiveness ratios for the incremental costs per QALY. Fifty-seven percent of the ratios were in the northwest quadrant revealing that family CBT is more costly and less effective compared to individual CBT, and thus inferior. Thirty- 
Graph 2a Cost-effectiveness plane for costs per anxiety-free child for family CBT versus individual CBT

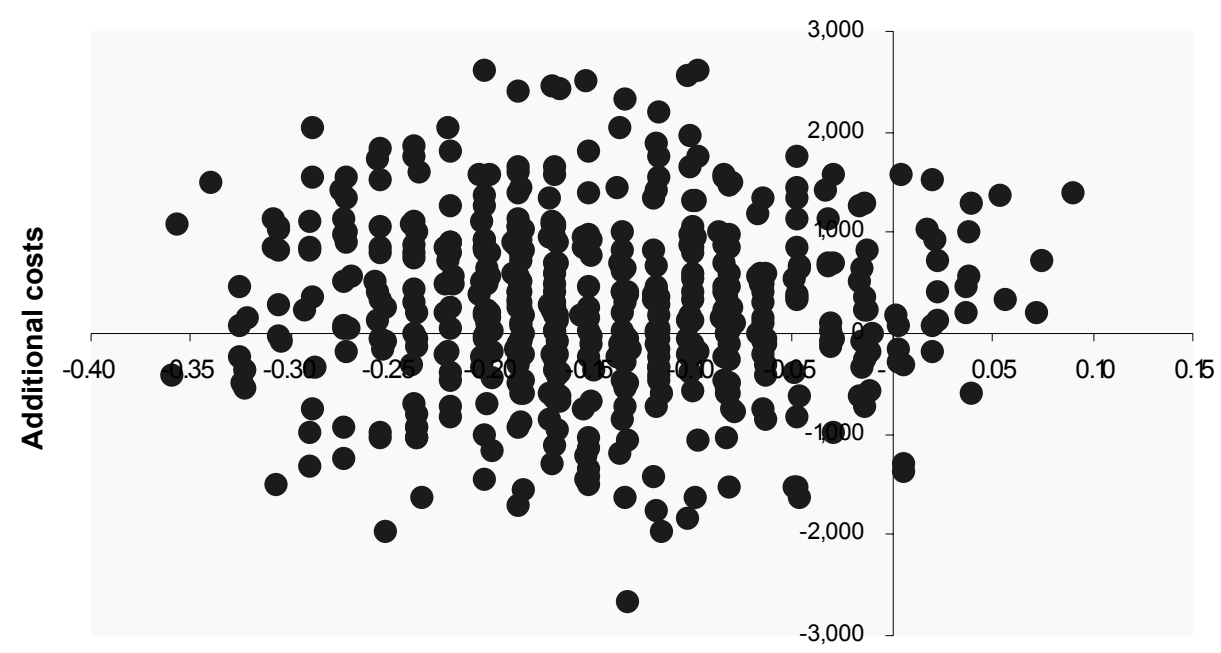

Additional effects

Graph $2 b$ Cost-effectiveness acceptability curve for the costs per anxiety-free child for family CBT versus individual CBT

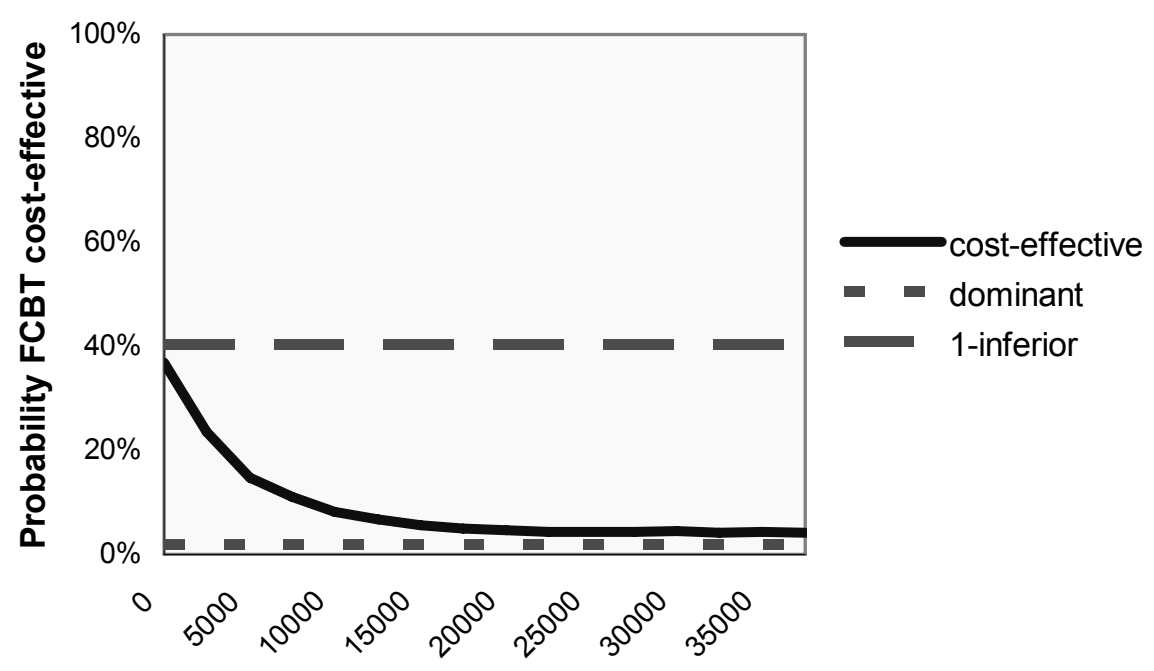

Ceiling ratio 
Graph 3a Cost-effectiveness plane for the costs per QALY for family CBT versus individual CBT

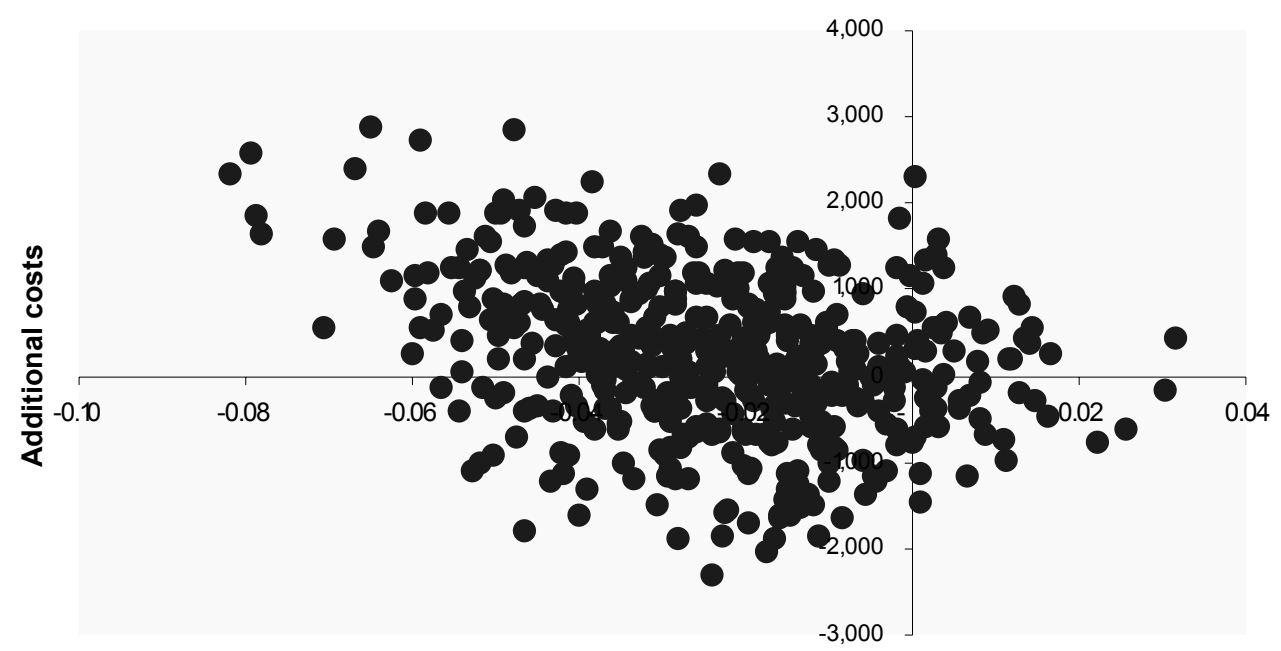

Additional effects

Graph 3b Cost-effectiveness acceptability curve for the costs per QALY for family CBT versus individual CBT

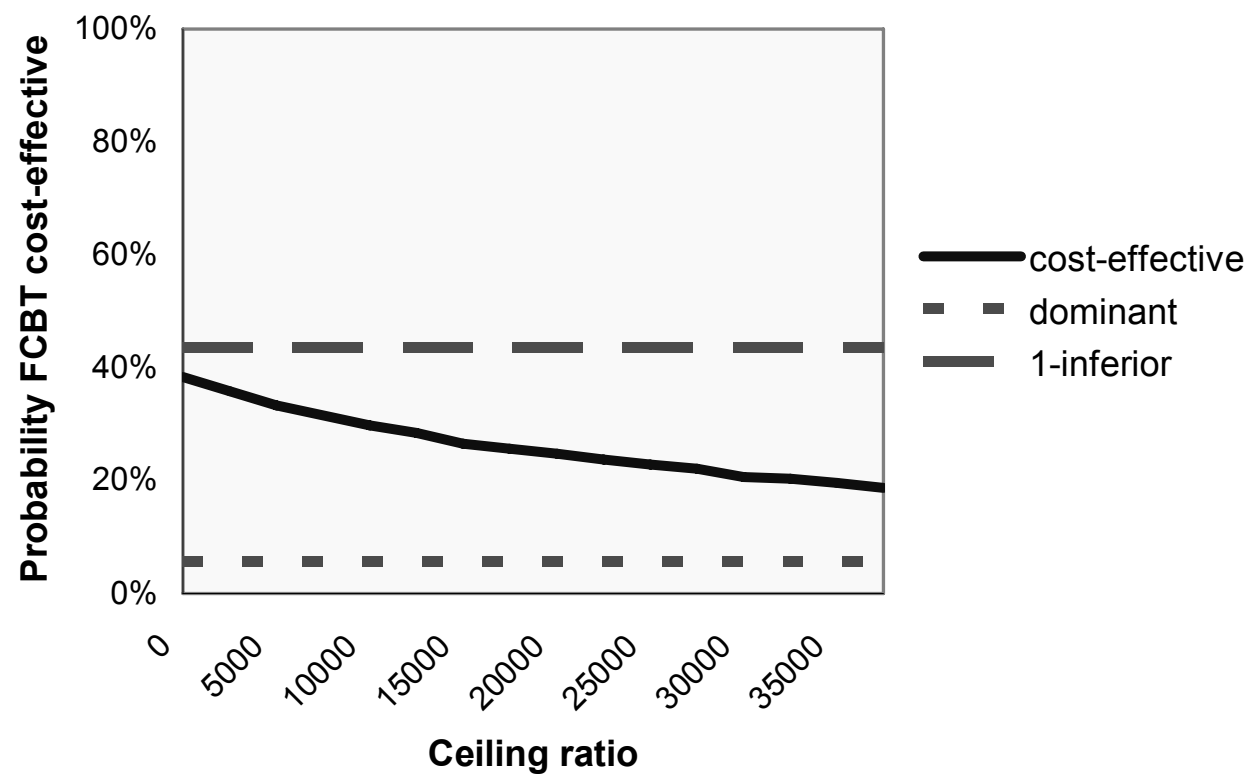


three percent of the ICERs were located in the southwest quadrant, which indicates lower costs and less QALYs. The northeast quadrant had 5\% of the ratios, showing more QALYs and higher costs. The remaining 6\% of the ICERs were in the southeast quadrant indicating lower costs and more QALYs.

Graph $3 b$ displays the acceptability curve for bootstrapped incremental costs per QALY. The probability that family CBT is cost-effective decreases from $36 \%$ to $19 \%$ by increasing the ceiling ratio from $€ 2500$ to $€ 37500$.

\section{Secondary cost-effectiveness analyses}

Five secondary analyses were conducted, of which results are presented in Table 5. In the first analysis, pre-treatment differences with respect to the utility values between family CBT and individual CBT, although not significant, were corrected for by using regression analysis, as recommended by Manca, Hawkins and Sculpher (2005). For each EQ-5D measurement, a regression analysis was conducted with the child's age and gender, treatment condition and pre-treatment utility value as independent variables. The dependant variable was the utility value per EQ-5D measurement (post-treatment, 3month follow-up, and 1-year follow-up). All analyses showed that the utility value at pre-treatment influenced the utility values over time, although the influence decreased. Per child, a corrected utility value per measurement was calculated by subtracting the pre-treatment utility value, multiplied with the corresponding beta, from the utility value per measurement. The corrected utility values were used to calculate corrected QALYs.

At pre-treatment, also cost differences were present between family and individual CBT. Analogous to the procedure described above, cost differences at pre-treatment were corrected for by means of regression analysis. All regression analyses showed that costs at pre-treatment influenced the costs over time, but the influence decreased. Per child, the corrected costs per cost diary were calculated by subtracting the pre-treatment costs, multiplied by the corresponding beta, from the costs per cost diary for that child.

In the second analysis, costs related to psychological problems of the child were included in the cost-analysis. In the main analysis, only anxiety related costs were included, based on subjective judgments by the parents. However, since anxiety is an internalizing disorder, it is possible that parents were not always able to interpret the symptoms correctly. Therefore, they may have attributed symptoms mistakenly to other psychological reasons, like depression, while in fact they were an expression of anxiety. Therefore, costs due to psychological reasons were also included in the cost-analysis.

The third analysis concerned the cost-effectiveness per anxiety-free family, by including not only the costs related to anxiety of the child but also costs related to anxiety of other family members. In order to be considered as an 'anxiety-free family', father, mother, anxious child and siblings had to be anxiety free on the ADIS at 1-year follow-up. 
The fourth and fifth analyses were sensitivity analyses to test the robustness of the results. The fourth analysis concerned the cost price for one hour of school absence, which was based on parent contributions and grants from the government. However, continuing absence from school may result in loss of a whole school year, and, as a consequence, starting a career later in life. Therefore, costs due to school absence were put on par with productivity costs due to loss of unpaid work, which is $€ 8.30$ per hour (Oostenbrink et al., 2005). Sixth, the cost price of day treatment was based on national reimbursements. Currently, all health care institutions are performing cost price calculations in light of a new Dutch financing system based on diagnosis-treatment combinations. Preliminarily results suggest that the actual cost prices are higher than the national reimbursements. Therefore, the cost price of day treatment was raised with $50 \%$, resulting in $€ 144$ per child per day (see Table 5).

All secondary analyses revealed that family CBT remained more costly and less effective, indicating inferiority for family CBT. Hence, results with respect to cost-effectiveness were highly robust for changing several parameters.

\section{Discussion}

To our knowledge, this is the first cost-effectiveness study in children with anxiety disorders. The cost-effectiveness analysis was conducted according to recently published international guidelines for trial-based economic evaluations (Ramsey et al., 2005). Although both societal costs and effectiveness were not statistically significantly different between family CBT and individual CBT, the point estimates of the cost-effectiveness ratios, expressing the costs per anxietyfree child and the costs per QALY, both indicated dominance for individual CBT. These results were confirmed by the bootstrap analyses, showing for both effectiveness measures that the largest part of the bootstrapped ICERs was in the quadrant where family CBT is inferior. Based on the bootstrapped results, the cost-effectiveness acceptability curves indicated that family CBT is not costeffective compared to individual CBT, irrespective of the chosen ceiling ratio. Finally, several secondary and sensitivity analyses revealed that the costeffectiveness results were robust. Therefore, it can be concluded that family CBT is not a cost-effective treatment, compared to individual CBT.

With respect to the effectiveness of CBT as measured by the ADIS, both treatments were highly effective (individual CBT $68 \%$ and family CBT 53\%) and compare well with an overall remission rate of $57 \%$ in a meta-analysis of CBT for childhood anxiety disorders (Cartwright-Hatton et al., 2004). Note that some studies included in this meta-analysis recruited children predominantly by means of advertisements, who are less severe anxious and therefore may easier meet the stringent criterion of free of all anxiety disorders, and did not include dropouts. The finding that family involvement did not add to the effectiveness of individual CBT is consistent with a recent meta-analysis showing no 
difference between child CBT and family focused CBT (In-Albon \& Schneider, in press). Also, effectiveness as measured by the QALY was not significantly different between individual and family CBT. However, utility values increased with 0.08 and 0.11 for respectively individual and family CBT, showing improvement in health-related quality of life for both groups. These improvements exceed the minimally important difference of 0.03 (Marra et al., 2005) of the EQ-5D, indicating that this instrument is sensitive to changes in anxiety.

Although health-related quality of life improved after CBT, the impact of children's anxiety on general quality of life seems marginal, considering that utility scores at pre-treatment were relatively high in both groups. This can be explained by the fact that parents reported mainly problems on the 'daily activities' and 'mood' dimensions. Also, only $6 \%$ of the anxious children had major problems on the mood dimension, while $50 \%$ had some problems and $44 \%$ had no problems on the mood dimension at pre-treatment. The fact that $44 \%$ reported no problems on the EQ-5D 'mood' dimension can possibly be explained by the fact that the definition of this dimension contains both anxiety and depression. In this study, only 5\% children had co-morbid depression. Moreover, the Dolan transformation puts the least of weight on the 'mood' and 'daily activities' dimensions, as a consequence of which utility values were higher than expected. Finally, the relatively high utility values can also be explained by a ceiling effect, which has been found frequently in studies using the EQ-5D in relatively healthy populations (Brazier, Roberts, Tsuchiya, \& Busschbach, 2004; de Wit, Busschbach, \& de Charro, 2000). Furthermore, low pre-treatment EuroQol scores possibly might have restrained further improvement in quality of life.

It is difficult to relate this study to other studies, because to the authors' knowledge no studies have been conducted on the costs and/or costeffectiveness of anxiety disorders in children. This study can only be related to studies on anxiety disorders in adults. However, after reviewing the literature, several differences were found. First, study populations consisted of adult patients with only one specific anxiety disorder (Heuzenroeder et al., 2004; McCrone et al., 2004). Second, treatment comparisons were made between CBT and drug interventions or optimal treatment (Issakidis, Sanderson, Corry, Andrews, \& Lapsey, 2004; McCrone et al., 2004). Also the effectiveness measures differed from the ones used in our study (Heuzenroeder et al., 2004). Therefore, results from our study cannot be compared to results from the published literature. Future research on the cost-effectiveness of CBT treatments for clinically anxious children may use our results as a "benchmark".

This cost-effectiveness study has some limitations that should be addressed. First, no care as usual condition was included in this costeffectiveness analysis. Care as usual in the Netherlands for childhood anxiety disorders is highly diverse but the field is moving rapidly towards applying CBT, although not always according to protocol. Moreover, in a meta-analysis 
Weiss, Catron, Harris, and Phung (1999) evaluated the effectiveness of usual psychotherapy as provided to children in outpatient clinics. The overall effect size was -.08 indicating no effectiveness. In contrary, the overall effect size for CBT based on 21 randomized control trials was 84 (In-Albon \& Schneider, in press). Hence, it is expected that a standardized CBT is more cost-effective than care as usual.

The time horizon in this study was only 15 months and therefore this study only provides an indication of the short-term cost-effectiveness of individual CBT. However, since the difference in efficacy and costs between individual and family CBT persisted over time, it seems likely that the superior cost-effectiveness of individual CBT will persist in time. Nevertheless, future follow-up assessments are necessary to establish the long-term costeffectiveness of individual CBT.

In conclusion, at short-term individual CBT is a cost-effective treatment for clinically anxious children seeking treatment. As health care costs associated with child anxiety are substantial (Polder et al., 2002), cost-effective treatments are necessary to reduce both anxiety and costs. Implementation of a standardized and efficacious individual CBT in daily practice seems therefore warranted. 


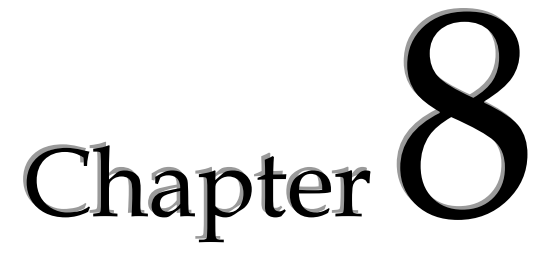

Discussion 
Anxiety disorders are the most common psychiatric disorders in children, are often combined with comorbid disorders such as depression and externalising disorders, cause interference with daily functioning of the child and without an effective treatment may worsen and even continue into adulthood (Keller, et al., 1992; Kendall, 1994). Hence, an effective treatment seems warranted. The majority of research shows that CBT is the only efficacious treatment for children with anxiety disorders (e.g. Cartwright-Hatton, Roberts, Chitsabesan, Fothergill, \& Harrington, 2004; In-Albon \& Schneider, in press).

In this thesis a new family based CBT was evaluated (Bögels \& Siqueland, 2006). Between January 2002 and December 2005, a randomized controlled trial was conducted to investigate effectiveness and costeffectiveness of family CBT versus individual CBT. In total, 146 families were included, of which 12 dropped-out before treatment and 6 were assigned to the waitlist condition and received treatment as usual afterwards. The remaining 128 families were randomized to individual CBT $(n=64)$ or family CBT $(n=64)$.

\section{Main findings}

The main findings of this thesis are as follows. First, chapter 2 shows that the Dutch societal costs of illness in families with a clinically anxious child, using the bottom-up approach amounted to approximately 20.3 million euros. This is almost 21 times as high compared to a matched sample of families from the general population. Second, in chapters 3 and 4 it was found that the Screen for Child Anxiety Related Emotional Disorders (SCARED-71) child and parent version and the Children's Automatic Thoughts Scale (CATS) differentiated between clinically anxious children and children from the general population, as a result, reliable cut-off scores were obtained. Third, chapter 5 reveals that there is growing evidence in the literature that parental involvement in child CBT is not more effective than solely individual CBT, but that the effects of family CBT have hardly been investigated. Fourth, the effectiveness study described in chapter 6 showed that, in contrast to the hypothesis, individual CBT was more effective than family CBT. Both treatments were less effective if parents suffered from anxiety disorders themselves and were more effective in younger children. Fifth, chapter 7 describes the cost-effectiveness study, in which the cost-effectiveness ratios, expressing incremental costs per anxietyfree child and per Quality Adjusted Life Year (QALY), showed that individual CBT dominated family CBT, in that associated costs were lower and effectiveness was higher for individual CBT. In this chapter, the findings of each chapter are discussed and recommendations for clinical practice and future research are made. Finally, the overall conclusion will be provided.

\section{Cost-of-illness}

To the author's knowledge, the cost-of-illness study, described in chapter 2 of this thesis, was the first study to examine costs in clinically anxious children. In addition to health care costs, non-health care costs (e.g. day care or informal 
care), and out-of-pocket costs (own contributions), also indirect costs (school absence and productivity loss of parents) were assessed. Interestingly, the results showed that next to health care costs, productivity loss of the parents and school absence of the child contributed mostly to the total costs. This indicates, first, that children with a clinical anxiety disorder suffer from a highly disabling disorder that prohibits them from fully engaging in school activities. This might lead to future societal problems like entering the labour market later in life or even unemployment as an adult. Second, not only does anxiety hinder the child in its' daily functioning, the parents also bear the consequences, in such a way that their presence at work diminishes due to the anxiety of the child.

Results in chapter 2 also indicated that bottom-up acquired total societal costs (health care, non-health care, out-of-pocket and indirect costs) for families of a clinically anxious child, referred for treatment, are 20.3 million euro in the Netherlands. Since anxiety disorders in children tend to last into adulthood (Newman et al., 1996), children probably continue to generate costs later in life due to their anxiety. Indeed, it was found that health care costs due to anxiety disorders in children and adults amounted to 180 million euro in 1999 (Polder, Takken, Meerding, Kommer, \& Stokx, 2002), whereas the health care costs in the cost-of-illness study described in chapter 2 amounted to 10.3 million. Since the latter study showed that societal costs are twice as high, societal costs in children and adults are assumed to be even higher than 180 million euros. Even more, costs associated with anxiety and other not anxiety related reasons are 21 times as high in families with clinically anxious children compared to families from the general population, indicating that societal costs for families with a clinically anxious child are huge. Therefore, an effective treatment for children with severe anxiety disorders seems warranted, not only to prevent future anxiety problems but also to reduce the corresponding high societal costs.

A cost-of-illness study measures the magnitude of the societal costs related to a specific (mental) illness. To obtain an estimation of this magnitude, two methods can be used namely the bottom-up and the top-down approach. In this study, the bottom-up approach was used which acquires patient data by means of registrations or self-report measures like cost diaries. Multiplying the costs per patient by the prevalence rate results in the total cost-of-illness for a specific (mental) illness. However, the investigated sample used to measure the cost-of-illness has to be representative for the entire sample of children with anxiety disorders. Since this study only examined costs in families with clinically anxious children (9.7\%; Verhulst, Van der Ende, Ferdinand, \& Kasius, 1997) referred for treatment (3.5\%; Verhulst \& van der Ende, 1997), thus $3.5 \%$ of $9.7 \%$, the results of our study cannot be generalised to all children with anxiety disorders, of whom many are not referred to mental health care. The latter group is estimated to be much larger $(96.5 \%$ of $9.7 \%)$, suggesting that the total societal costs of children with anxiety disorders might be much higher. 
Although the total societal costs might be higher if all anxious children were included in the cost of illness calculation, the annual societal costs per child might be lower compared to our estimation due to the high prevalence rate. On the other hand, the children with anxiety disorders that are not referred to mental health care may receive more other forms of help such as medical care, as the results of our top-down approach suggest. The costs of such care may be as high or even higher as that of mental health care. Future research should therefore investigate the costs of children with anxiety disorders in the whole range of the severity spectrum of anxiety disorders, whereby variation in use of mental health care and other facilities such as medical care can be determined.

The costs of families with clinically anxious children were almost 21 times as high compared to costs of families from the general population. A large part of this difference could be allocated to anxiety related costs, showing good discriminative validity of the cost diary. However, significant differences were also found on costs due to other reasons. This could be caused by the usage of the prospective diary, in which parents were asked to record the reasons for resource use and might mistakenly have attributed costs to other non-anxiety reasons. Therefore, as an alternative, costs could be obtained through retrospective cost interviews. This method allows the interviewer to ask exhaustive questions to be sure that all anxiety related costs are included and not ascribed to other reasons.

Although good discriminative validity and convergent validity were established for the prospective cost diary used in the cost-of-illness study (chapter 2), the criterion validity of the cost diary was not established. Criterion validity would provide us with a conclusive answer regarding the true societal costs of illness of clinically anxious children. Criterion validity can be established by comparing actual resource use based on formal institutional registrations with reported resource use in the cost diaries. However, this would require a detailed inventory, by consulting the records of the 118 clinically anxious children and their parents at general practitioners, hospitals, mental health institutions, schools and employers, which is very time consuming and difficult. Nevertheless, based on good discriminative validity and convergent validity, our cost of illness results obtained with prospective cost diaries seems a good approximation of the true costs in clinically anxious children in need of treatment.

\section{Effect measures: Anxiety and cognition measures}

Effectiveness studies usually use interviews and questionnaires to measure changes in anxiety symptoms and/or diagnostic status. In this thesis improved assessment methods for change in anxiety symptoms and cognition were used. In order to obtain these qualified measures, new questionnaires were developed. First, the existing 66-item Screen for Child Anxiety Related

Emotional Disorders (Muris, Merckelbach, Schmidt, \& Mayer, 1999) did not sufficiently measure social phobia, therefore this anxiety questionnaire was 
enlarged by adding 5 extra social phobia items. The validity of this altered SCARED-71 as a screenings instrument is described in chapter 3. Second, the Child Automatic Thoughts Scale, (CATS; Schniering \& Rapee, 2002) examined in chapter 4 was translated into a Dutch version to measure negative automatic thoughts. Cut-off scores for these two questionnaires were obtained by differentiating between clinically anxious children and children from the general population. These cut-off scores can be employed in prevention research for example to identify children at high risk and in effectiveness studies to determine whether the treatment has been successful in such a way that a child has reached a subclinical level of anxiety symptoms and negative automatic thoughts. Cut-off scores might be very relevant for clinical practice in such a way that therapists have a bench-mark to evaluate the end state functioning of the child by using a questionnaire instead of an elaborate interview.

Unique was that the CATS was assessed to examine whether cognitions change as well after the child receives CBT. Earlier treatment studies often failed to investigate the change in cognitions. In the effect study described in chapter 6, moderate effect sizes were found for the CATS, indicating that both individual CBT and family CBT did change cognitions. Besides, $72 \%$ of the children in the individual CBT condition and $62 \%$ of the children in the family CBT condition fell into the normal range of having negative automatic thoughts after treatment. These results show that the CATS is able to detect change in cognitions after the treatment of an anxious child.

\section{Effectiveness}

The main effectiveness study, described in chapter 6, showed that individual $\mathrm{CBT}$ is more effective than family CBT. Also when reviewing the literature on parental involvement, the evidence grows that parental involvement is not more effective compared to treating the child alone (see chapter 5). It should be mentioned that both therapies are effective in treating children with anxiety disorders. At 3-month follow-up, only 5\% $(n=3)$ of the children in the individual CBT condition and $17 \%(n=11)$ of the children in the family CBT condition did not benefit from the treatment, in other words the severity of their primary anxiety disorder did not decline. It should also be mentioned that $19 \%$ $(n=12)$ of the families receiving family CBT dropped out, that is, did not complete the 12-session family CBT or exceeded the number of sessions as required by the protocol. Reasons for dropout during family CBT were; rapid improvement (1), lack of motivation (2), depressed and suicidal child (2), language problems (1), severe illness mother (1), dyslexia in mother and child (1), too much pressure on anxious father (1) and extra sessions due to severe child psychopathology (3). Based on reasons for dropout, it could be concluded that it is more difficult to get the family into family CBT due to psychopathology of the child and motivational problems. Due to the finding the family CBT is lees effective than individual CBT and the higher percentage of 
treatment dropouts, it can be concluded that family CBT has quite a few catches.

To further investigate the reason for dropouts in de family CBT condition, pre-treatment differences between family CBT completers versus dropouts were carried out. The only difference was found on the percentage of broken homes, which was higher $(36 \%, n=4)$ in family CBT dropouts than in family CBT completers $(14 \%, n=7)$. Furthermore, in the family CBT dropout group, $73 \%(n=8)$ of the mothers were housewives, which was significantly higher than the percentage of housewives in the completer group $(28 \%, n=14)$. It could be concluded that more complexity in families and less paid employment of mothers occurred to be associated with family treatmentdropout. A highly speculative explanation is that being a housewife is associated with more overprotection of the anxious child and more resistance to change. More research is necessary to understand the association between broken homes and less paid employment of mothers with treatment-dropout during family treatment.

Furthermore, the influence of age and parental anxiety as moderators was investigated in chapter 6 . The hypothesis that family CBT would be more effective in young children whereas child CBT would be more effective in adolescent children was not confirmed. However, a more general effect was found namely; CBT is more effective in 8- to 12-year-old children compared to 13- to 17-year-old adolescents. First, this effect could be explained by the fact that the workbooks used during the treatments were not developmentally sensitive. That is, children aged 8 to 18 received the same workbook. Developmentally sensitive workbooks tailored to the child's cognitive and emotional development might enhance the effects of future treatment. A second explanation for the age effect is that autonomy is very important for adolescents in other words they want to be independent of parents and other grown-ups. This may cause them to react against their parents as well as the therapist and to be less cooperative with the therapist, so therapeutic alliance is more difficult to establish (Hudson, Kendall, Coles, Robin, \& Webb, 2002). Third, adolescents may have established more rigid and persistent negative cognitive and behavioural patterns (e.g. avoidance) due to the longer duration of anxiety symptoms in comparison with younger children. Therefore, it might be more difficult to change these patterns with CBT.

Whatever the explanation for the superior effects of CBT at a younger age, the results do suggest that it is better to treat children when they are younger (8-12). This finding may have important implications for prevention and treatment. As young children often do not self-refer for treatment, it is important that those who may refer children, such as general practitioners, school counsellors, and parents, are aware of the good results of treatment at a young age, and therefore do not postpone referral. Also, from a prevention point of view, it may be that the ideal age to offer children who are at risk for 
developing anxiety disorders (or already have one) may be between 8-12 rather than later.

Also, in contrast to the hypothesis, family CBT was not more effective when parents suffered from anxiety disorders themselves. In contrast, this study showed that CBT in general is less effective if parents suffer from anxiety disorders themselves. This indicates that anxious parents might hinder the child's treatment. Hence, it could be suggested that anxious parents have to be treated for their own anxiety first, before the child can receive CBT in order to enhance treatment success. However, since anxiety disorders are also partially genetically transmitted (for a review and meta-analysis see Hettema, Neale, \& Kendler, 2001), children of anxious parents might have an innate sensitivity which makes treatment more difficult to alter anxiety, while a child that acquired its' anxiety through non-genetic causes, such as conditioning, might be more easily treated.

Although the findings in this thesis indicate the superiority of individual CBT over family CBT, some precaution should be made before drawing this conclusion. The Task Force on Promotion and Dissemination of Psychological Procedures (1995) has proclaimed that in order to be a probably effective treatment, treatments must be efficacious in at least two randomized control trials in which the treatment is compared to a wait-list or control condition. Therefore, a second randomized control trial should be conducted in order to be able to conclude that both treatments are effective and to show superiority of individual CBT over family CBT.

Also of importance is the patient preference, which was not obtained in our effectiveness study. In the effectiveness study described in chapter 6, families were randomized to either individual or family CBT. Perhaps families should decide themselves which treatment they want or prefer. From our study, it is unclear whether families would have preferred one treatment above the other. Future research should address this question.

\section{Cost-effectiveness}

In chapter 7 of this thesis, the first cost-effectiveness study in clinically anxious children was described. Until now, no study investigating the effects of CBT has taken costs into account as well. Results from our cost-effectiveness study showed that although costs and effects did not differ significantly between individual CBT and family CBT, cost-effectiveness ratios expressing incremental costs per anxiety-free child and incremental costs per Quality Adjusted Life Years (QALY), showed that individual CBT was dominant and hence more cost-effective than family CBT. Effectiveness was measured based on the percentage of anxiety free children and on the number of QALYs of the child.

Health related quality of life was measured with the EuroQol child and parent version (Stolk, Busschbach, \& Vogels, 2000). This was the first study to assess health related quality of life by means of child self-report, maternal and 
paternal report. Due to the high correlation between child and parent reported quality of life, only parent scores were aggregated and used in the costeffectiveness analysis. A literature study on 3,050 quality of life articles in children found that only in $9 \%$ of the articles, children were involved in the evaluation of quality of life (Wallander, Schmitt, \& Koot, 2001). Thus usually only the proxy or parent report of the child's quality of life is obtained because it is believed that children lack the language skills, cognitive abilities and a long term view of events to report their own quality of life (Stolk et al., 2000). However, adult studies have shown that proxy reports often underestimate quality of life of the patient. In addition, internalising disorders such as anxiety disorders are very subjective which makes it more difficult for a parent to report quality of life (Wallander et al., 2000). As a result, most studies have found that the parent-child agreement is low to moderate on both generic quality of life instruments (e.g. the EuroQol) and specific quality of life instruments (e.g. the SCARED). The high parent-child agreement found in chapter 7 is therefore remarkable. Given the inconsistency between the poor parent-child agreement in the literature and the high agreement found in chapter 7, future research should address this issue. It could be suggested that both child and proxy version have to be assessed to obtain more accurate and complete information about the quality of life of the child. If the agreement is high, either one utility score can be used in the cost-effectiveness analysis, if not, two different cost-effectiveness analyses should be performed.

Furthermore, this study is the first to measure change in generic health related quality of life in children after treatment. Until now, only 11 adult studies have been conducted that measured generic quality of life before and after treatment in patients with panic disorder $(n=8)$, social phobia $(n=2)$ and posttraumatic stress disorder $(n=1)$, however the majority of these studies concerned psychopharmacological interventions (Mendlowicz \& Stein, 2000; Meltzer-Brody \& Davidson, 2000). It is more than logical to obtain generic quality of life in psychopharmacological studies, since pharmaceuticals often have physical side effects, which can intervene enormously with quality of life. However, it is also useful to assess quality in life in CBT studies since generic quality of life questionnaires are commonly used in economic evaluations to obtain utility scores. Even more, our data show that generic quality of life improved after CBT in both groups. These improvements exceeded the minimally important difference, suggesting that the generic quality of life instrument used in our study, the EuroQol, is sensitive to changes in anxiety. Therefore, besides using specific quality of life questionnaires such as anxiety or psychopathology questionnaires, future studies should use generic quality of life instruments that cover all relevant aspects of health related quality of life before and after treatment.

Future research should address cost-effectiveness of treatment in children with anxiety disorders in general, since this study only showed the cost-effectiveness of individual CBT in clinically referred children. Perhaps, 
offering an effective short-term intervention to all children with anxiety disorders might reduce societal costs and might prevent children from fully developing a clinical anxiety disorders. Especially since young children benefit more from CBT, this age group should be the target. As chapter 2 of this thesis shows, children with clinical anxiety disorders cost 21 times more than children from the general population, including nonclinically anxious children. Possible future costs of treatment and the consequences of clinical anxiety disorders might be saved by providing an effective treatment. Taken this even further, prevention programs might reduce costs of anxiety disorders as well. Children at risk for anxiety disorders can be traced with screening instruments and are offered a short intervention. This prevents children from developing a severe anxiety disorder that in the long term might cause high cost due to mental health and other facilities, as shown in our cost-of-illness study. A study by Simon, Bögels and Dirksen (personal communication) currently addresses the cost-effectiveness of a prevention program. This study uses the same assessments like the EuroQol and the prospective cost diary described in this thesis. Therefore, results of this study regarding the cost-effectiveness of the prevention program can be easily compared to our results, to see whether it is more cost-effective to provide a short intervention to children at risk than to offer effective treatments to children with a severe anxiety disorder who seek help in mental health care. Eventually, conclusions can be drawn on when and how anxious children should be treated to obtain optimum effectiveness and cost-effectiveness.

\section{Treatment implications}

How family CBT principles could be used: Modular CBT

Although family CBT was found to be less effective than individual CBT, it could still have benefits in an altered form. It could be suggested that modules should be made, that can be used according to the needs of the family (component selection). These modules should be used in addition to individual $\mathrm{CBT}$, which has proven to be very efficacious (see chapter 6). When there are problems concerning family functioning, extra modules should be provided by the therapists to address these problems. If parents are anxious they should be treated as well, using a specific module. However, if there are no family functioning problems or if the parents do not have any kind of psychopathology, the designated module should not be addressed. Individual CBT should therefore be complemented with several modules, which can be used according to the needs and problems of the family.

In order to get insight in the problems and needs of families, an extended intake and elaborate assessment of family functioning should be obtained before entering treatment. Then, the therapist can decide which modules to use to address the specific family problems (therapist selection). It could also be suggested that families themselves choose the modules, according 
to their own needs (patient selection). By means of tailoring individual CBT based on needs of the child and family, effectiveness may be increased. Preliminary support for a modular based treatment was found in a pilot study by Chorpita, Taylor, Francis, Moffitt and Austin (2004). Their modular CBT consisted of a combination of individualised scripted techniques matched to the child's needs and strengths. Four core CBT elements were provided to all 7 children that is self monitoring, psycho education, exposure and maintenance and relapse prevention. Additional modules were given based on decision rules provided in a flowchart and included, cognitive restructuring, social skills training, rewards, reinforcement strategies and time-out. Of the 7 referred anxious children, 6 were free of all anxiety disorders at 6-month follow-up. All 7 were free of their primary diagnosis at 6-month follow-up. However, this latter study only used CBT modules and not more specific modules like family functioning. Furthermore, the sample size was small and no comparison was made with a more stringent or standard CBT. Future research could investigate whether a tailored and modular individual CBT, including family CBT elements, is more effective than standard individual CBT.

\section{Flexible number of sessions}

In the effectiveness study, it was found that immediately after CBT, $41 \%$ of the children still had an anxiety disorder. This could be due to the limited number of sessions that were obliged in the used protocol. Perhaps if more sessions were given, more children would be free of all anxiety disorders, since the therapist would have more time targeting the primary anxiety disorder more profoundly and other anxieties as well. Especially in family CBT, extra sessions might be necessary, since so many targets are set in this therapy; both the child's anxiety and the parents' anxiety is targeted, distorted cognitions of child and parents are identified and altered, family functioning is dealt with, and parents' communication is improved. With extra sessions these themes can be fully exploited. This is confirmed by the fact that 3 families in family CBT needed more than 12 sessions and eventually were excluded because of protocol violation.

More sessions could improve the percentage of children free of anxiety disorders but the reverse can also be effective; fewer sessions can be enough for some children. In the effectiveness study, one child dropped-out because after 4 sessions it was free of anxiety disorders. Some other children also mentioned that they made so much progress they thought fewer sessions would be enough, however they completed the treatment because of the protocol requirements. Furthermore, Ost, Svensson, Hellström, and Lindwall (2001) showed that a 1-session exposure with or without the parent present was more effective than a waitlist condition in children with phobias, thus even one session makes a difference. Even more, a modular CBT by Chorpita et al. (2004) found that the sessions delivered to obtain effectiveness ranged from 5 to 17, thus also providing evidence for an effective individual CBT with flexible 
number of sessions. However, long-term effects of short interventions have not been investigated yet. In conclusion, CBT could increase in effectiveness if the number of sessions is adapted to the needs of the child. This can be obtained by asking the child and parents if they want to continue (child or family preference) but could also be obtained by means of an objective standard. Examples of objective ways of assessing the need for further treatment are a short anxiety interview to re-assess the anxiety disorder status, or readministration of the SCARED to establish whether the child has reached an anxiety symptom level below the cut-off score. Such an objective assessment could be administered every session or every group of sessions.

Not only is a more flexible number of sessions ideal for the anxious child and its' family, it may also be more cost-effective at the long term. If a treatment is terminated after 12 sessions, while the child is still anxious, the child might relapse or continue to have problems into adulthood, causing more costs. The reverse, when a child continues the remaining treatment sessions of a structured protocol, although the anxiety is already gone, is also cost inefficient. The sessions (and money), this latter child spares can be re-allocated to the anxious child who needs more sessions, which may cause an equilibrium. Hence, modifying the number of sessions to the child needs may be more costeffective in the long run. Future research should address this matter.

\section{Future research}

\section{Moderators and mediators}

In the effectiveness study it was found that $52 \%(n=66)$ of the children was free of all anxiety disorders and in $89 \%(n=114)$ at least the primary anxiety disorder decreased in severity at 3-month follow-up. However, the anxiety disorders of 5 children that completed the treatment (2 individual CBT and 3 family CBT) did not improve. Even more, it is assumed that 9 children that dropped-out of treatment did not improve as well, however last assessment carried forward was used due to lack of assessments, thus this could only be suggested based on oral therapist reports. It can be concluded that some children respond better to CBT than others. Insight in possible child or parental characteristics that are associated with the effectiveness of CBT, the so-called moderators, have the potential to further refine CBT and to enhance its' effectiveness. Moderators are variables that influence the direction and/or strength of the relationship between an independent variable (having an anxiety disorder) and a dependant variable (treatment outcome) (see diagram 1). Examples of moderators are socio-demographic factors like gender, ethnicity and age and diagnostic characteristics like primary anxiety disorder, severity and comorbidity. Also parental characteristics such as psychopathology, socioeconomic status and marital status could be related to success or failure of treatment. Studying moderators can lead to the finding; whom under which 


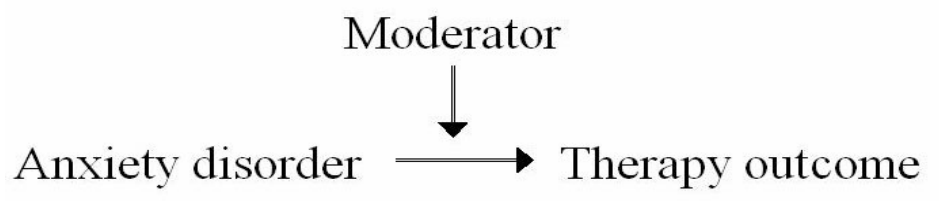

Diagram 1: Moderator effect

circumstances is most responsive to treatment (Kazdin \& Nock, 2003; Kraemer, Wilson, Fairburn, \& Agras, 2002).

Although, compared to individual CBT, family CBT was not more effective in younger children and if parents were anxious themselves (see chapter 6), family CBT could be more effective when investigating other moderators of influence. In other words, it could be suggested that for certain types of families, family CBT might possibly be more effective. For instance, family CBT seems an alternative for individual CBT when parents do not suffer from psychopathology.

In contrast to our findings, Crawford and Manassis (2001) did not find that parental psychopathology influenced treatment outcome, however the moderators family dysfunctioning and clinician and child report of parental frustration predicted a poorer outcome of individual or group CBT with parental involvement. In case there are problems in family dysfunctioning, these families might benefit more from family CBT because this treatment focuses on family problems.

Research on mediators is necessary to explain the possible association between child or parental characteristics and effectiveness. Mediators are mechanisms through which the independent variable (having an anxiety disorder) influences the dependant variable (treatment outcome) (see diagram 2). In other words, through which process does the therapeutic change occur (Kazdin \& Nock, 2003; Kraemer et al., 2002)? For example, age is considered as a moderator since it cannot be changed by treatment, however, the cognitive level that is associated with age is a mediator because CBT can influence the cognitive process and thereby can influence the outcome of treatment. Another mediator is therapeutic alliance. In Holland, a multi center study of the University of Amsterdam (Clinical child and youth psychology), Amsterdam Medical Centre (de Bascule) and Accare Child and youth psychiatry Groningen by Prins and de Haan, (personal communication) and a study by Heyne, van Hout, Maric, and Sauter (personal communication) in Leiden are currently conducting a research to investigate mediators and moderators in anxious children and children with school refusal respectively.

In conclusion, child and parental characteristics can be used to adapt treatments. In case a child is at risk for treatment failure due to several characteristics and parental psychopathology, the therapist can anticipate on these factors. Future research should address moderators and mediators to 


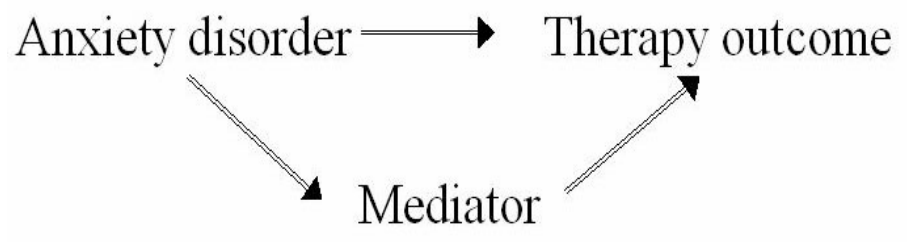

Diagram 2: Mediator effect

expand our knowledge of anxiety disorders and to enhance effective treatments. Methodological and statistical guidelines to study moderators and mediators can be found in Kazdin and Nock (2003) and Kraemer et al. (2002).

\section{Mechanisms of change}

A lot of research has been conducted on the effectiveness of several CBT programs. However, it is still unclear which component of CBT is the most effective part or which process causes therapeutic change. Kendall et al. (1997) executed a treatment study in which CBT was divided in two periods of sessions, a first part and a second part. The content of the parts was balanced between the two periods and included exposure based sessions and educational support based sessions. They found that if exposure was given in the second part of CBT, effects increased enormously compared to the educational support in the first part alone. If education support was given in the second part of treatment, the effects remained the same compared to the exposure sessions in the first part. The finding that exposure is indeed an important feature of CBT was confirmed by Ost et al. (2001). They found that a one-session exposure reduced anxiety complaints. However, an exposure based contingency management (child exposure and parental behavioural strategies) was less effective than an exposure based cognitive self-control (child exposure, cognitive strategies, self-evaluation and self reward and parental training on child's self control skills) (Silverman et al., 1999). Last, Hansen and Franco (1998) and Silverman et al. (1999) both found that a non-active educational control condition was as effective as CBT. Due to these inconclusive results, future research is necessary to study the mechanisms of CBT that contribute to effectiveness.

\section{Care as usual and decision making}

In order to make a statement about whether individual CBT is most effective or cost-effective in general, it is necessary to compare individual CBT with other existing treatments for children with anxiety disorders such as psychopharmacological interventions, cognitive interventions, behavioural interventions, psychodynamic interventions, Eye Movement Desensitisation and Reprocessing (EMDR) and Relaxation Therapy. However, in clinical 
practice a lot of variations and combinations of these aforementioned treatments are provided. Due to this diversity of treatments, it is impossible to refer to a treatment as "care as usual". However, care as usual for childhood anxiety disorders as it is currently given in the Netherlands is moving rapidly towards applying CBT, in multiple formats. However, so far, no study inventoried the range of existing treatments that are currently executed in clinical practice, thus it remains unclear what care as usual for children with anxiety disorders is.

Concerning effectiveness, in a meta-analysis of Weiss, Catron, Harris, and Phung (1999) the overall effect size of usual psychotherapy as provided to children in outpatient clinics was found to be -.08 indicating no effectiveness. In contrary, the effect size for individual CBT found in chapter 6 was 1.39 for change in anxiety symptoms. So, it could be suggested that individual CBT is more effective than care as usual.

Nevertheless, in order to provide an overall conclusion on effectiveness and cost-effectiveness, all possible alternative treatment types, including the diverse care as usual, should be included in the analysis. Although results of effectiveness and cost-effectiveness studies from randomised control trials are important and useful to determine effectiveness and cost-effectiveness for specific treatments, one RCT alone does not provide enough information about effectiveness and cost-effectiveness along all existing treatments. Therefore, the clinical and economic evaluation of treatment in children with anxiety disorders should be examined within a broader framework in which all evidence of available RCT's is accumulated and evaluated. This model can than generate a statement about effectiveness and cost-effectiveness which can lead to more profound decision making and resource allocation (Sculpher, Claxton, Drummond, \& Mcabe, 2006).

\section{Long-term follow-up}

A long-term follow-up study is necessary to investigate whether the conclusions drawn in this thesis still stand at 5 to 10 years from now. Our follow-up data suggest that results concerning effectiveness and costeffectiveness are stable throughout 3-month and 12-month follow-up, suggesting a stable pattern over time. Other effectiveness studies have shown a prolonged effect of CBT after 5 to 9 years follow-up (Barrett, Duffy, Dadds, \& Rapee, 2001; Kendall, Safford, Flannery-Schroeder, \& Webb, 2004). For example, Kendall et al. (2004) showed that of the $91 \%$ of the original sample, $90 \%$ of the children was (still) free of their primary diagnosis 5.5 to 9.3 years after treatment according to the child interview, which is quite a high percentage. However, no studies have addressed cost-effectiveness of CBT yet and therefore long term cost-effectiveness is also not examined yet. Future follow-up research should, beside effects, investigate the long-term costs as well. 
Overall conclusion

Overall, it can be concluded that, given our results, individual CBT seems the best choice for treating children with clinical anxiety disorders, not only from the child's perspective (more effective), but also from a societal perspective (cost-effective). Also when reviewing the literature on parental involvement, the evidence grows that parental involvement is not more effective compared to treating the child alone.

Given the large effect size for individual CBT (see chapter 6), the high percentage of children free of anxiety disorders for individual CBT (see chapter 6) and the favourable results regarding the cost-effectiveness of individual CBT (see chapter 7), it can be concluded that for now, implementation of individual CBT in clinical practice seems warranted. However, given the many recommendations and suggestions for future research, it can be suggested that in the meanwhile, other research has to be done to investigate moderators, mediators, mechanism of change of CBT and long term effects and costeffectiveness regarding the full range of possible treatment options, in order to be able to optimize effectiveness and cost-effectiveness of treatments for children with anxiety disorders. 
References 
Achenbach, T. M., \& Edelbrock, C. S. (1983). Manual for the Child Behavior Checklist and Revised Child Profile. Burlington: VT: University of Vermont, Department of Psychiatry.

Ambrose, B., \& Rholes, W. S. (1993). Automatic cognitions and the symptoms of depression and anxiety in children and adolescents: An examination of the content specificity hypothesis. Cognitive Therapy and Research, 17, 289-308.

Ament, A., \& Evers, S. (1993). Cost-of-illness studies in health care: a comparison of two cases. Health policy, 26, 29-42.

American Psychiatric Association (1994). Diagnostic and statistical manual of mental disorders (4th ed.). Washington, DC: American Psychiatric Association.

Anderson, J. C., Williams, S., McGee, R., \& Silva, P. A. (1987). DSM-III disorders in preadolescent children. Archives of General Psychiatry, 44, 69-76.

Babyfun (Babypret). (2005). Retrieved 2005 from http://www.babypret.nl.

Bakker, F. C., van Wieringen, P. C. W., van der Ploeg, H. M., \& Spielberger, C. D. (1989). Handleiding bij de Zelf-Beoordelings-Vragenlijst voor kinderen ZBV-K; Een Nederlandstalige bewerking van de State-Trait Anxiety Inventory for children (STAIC) van Spielberger e.a. Lisse, Swets \& Zeitlinger B.V.

Barber, J. A., \& Thompson, S. G. (2000). Analysis of cost data in randomized trials: an application of the non-parametric bootstrap. Statistics in Medicine, 19, 3219-3236.

Barrett, P. M. (1998). Evaluation of cognitive-behavioral group treatments for childhood anxiety disorders. Journal of Clinical Child Psychology, 27, 459-468.

Barrett, P. M., Dadds, M. R., \& Rapee, R. M. (1996). Family treatment of childhood anxiety: A controlled trial. Journal of Consulting and Clinical Psychology, 64, 333-342.

Barrett, P. M., Duffy, A. L., Dadds, M. R., \& Rapee, R. M. (2001). Cognitive-behavioral treatment of anxiety disorders in children: Long-term (6-year) follow-up. Journal of Consulting and Clinical Psychology, 69, 135-141.

Beck, A. T., Brown, G., Steer, R. A., Eidelson, J. I., \& Riskind, J. H. (1987). Differentiating anxiety and depression utilizing the Cognition Checklist. Journal of Abnormal Psychology, 96, 179-186.

Beck, A. T., \& Clark, D. A. (1988). Anxiety and depression: An information processing perspective. Anxiety Research, 1, 23-36.

Beidel, D. C., Turner, S. M., \& Morris, T. L. (1995). A new inventory to assess childhood social anxiety and phobia: The Social Phobia and Anxiety Inventory for Children. Psychological Assessment, 7, 73-79.

Beidel, D. C., Turner, S. M., \& Morris, T. L. (2000). Behavioral treatment of childhood social phobia. Journal of Consulting and Clinical Psychology, 68, 1072-1080.

Bernstein, G. A., Borchardt, C. M., \& Perwien, A. R. (1996). Anxiety disorders in children and adolescents: A review of the past 10 years. Journal of the American Academy for Child and Adolescent Psychiatry, 35, 1110-1119.

Birmaher, B., Khetarpal, S., Brent, D., Cully, M., Balach, L., Kaufman, J., et al. (1997). The Screen for Child Anxiety Related Emotional Disorders (SCARED): Scale Construction and Psychometric Characteristics. Journal of the American Academy for Child and Adolescent Psychiatry, 36, 545-553.

Board of Health Insurances (College voor Zorgverzekeringen), Medication and aid Information Project (Genees- en hulpmiddelen Informatie Project, GIP databank) (2005). Retrieved 2005 from http://www.gipdatabank.nl.

Board Tariff rates Health care and Care authority (College Tarieven Gezondheidszorg Zorgautoriteit in oprichting). (2005). Retrieved 2005 from http:/ / www.ctg-zaio.nl.

Bögels, S. M., \& Brechman-Toussaint, M. L. (in press). Family Issues in child anxiety: Attachment, parental rearing, family functioning, and beliefs. Clinical Psychology Review.

Bögels, S. M., \& van Melick, M. (2004). The relationship between child-report, parent self-report, and partner report of perceived parental rearing behaviors and attitudes and anxiety in children and parents. Personality and Individual Differences, 37, 1583-1596.

Bögels, S. M., \& Siqueland L. (2006). Family cognitive behaviour therapy for children and adolescents with clinical anxiety disorders. Journal of the American Academy of Child and Adolescent Psychiatry, 2, 134-141. 
Bögels, S. M., Snieder, N., \& Kindt, M. (2003). Specificity of dysfunctional thinking in children with symptoms of social anxiety, separation anxiety and generalised anxiety. Behaviour Change, 20, 160-169.

Bögels, S. M. \& Zigterman, D. (2000). Dysfunctional cognitions in children with social phobia, separation anxiety disorder, and generalised anxiety disorder. Journal of Abnormal Child Psychology, 28, 205-211.

Brady, E. U., \& Kendall, P. C. (1992). Comorbidity of anxiety and depression in children and adolescents. Psychological Bulletin, 111, 244-255.

Brazier, J., Roberts, J., Tsuchiya, A., \& Busschbach, J. (2004). A comparison of the EQ-5D and SF-6d across seven patient groups. Health Economics, 13, 873-884.

Briggs, A. H., Wonderling, D. E., \& Mooney, C. Z. (1997). Pulling cost-effectiveness analysis up by its bootstraps: a non-parametric approach to confidence interval estimation. Health Economics, 6, 327-340.

Brooks, R. with the EuroQol Group (1996). EuroQol: the current state of play. Health Policy, 37, 5372.

Brown, T. A., DiNardo, P. A., Lehman, C. L., \& Campbell, L. A. (2001). Reliability of DSM-IV anxiety and mood disorders: implications for the classification of emotional disorders. Journal of Abnormal Psychology, 110, 49-58.

Bruijnzeels, M. A., Foets, M., van der Wouden, J. C., Prins, A., \& van den Heuvel, W. J. A. (1998a). Measuring morbidity of children in the community: a comparison of interview and diary data. International Journal of Epidemiology, 27, 96-100.

Bruijnzeels, M. A., van der Wouden, J. C., Foets, M., Prins, A., \& van den Heuvel, W. J. A. (1998b). Validity and accuracy of interview and diary data on children's medical utilization in the Netherlands. Journal of Epidemiology and Community Health, 52, 65-69.

Campbell, M. A., \& Rapee, R. M. (1994). The nature of feared outcome representations in children. Journal of Abnormal Child Psychology, 22, 99-111.

Cartwright-Hatton, S., McNally, D., \& White, C. (2005). A new cognitive behavioural parenting intervention for families of young anxious children: A pilot study. Behavioural and Cognitive Psychotherapy, 33, 243-247.

Cartwright-Hatton, S., Roberts, C., Chitsabesan, P., Fothergill, C., \& Harrington, R. (2004). Systematic review of the efficacy of cognitive behaviour therapies for childhood and adolescent anxiety disorders. British Journal of Clinical Psychology, 43, 421-436.

Caspi, A., Elder, G. H. Jr., \& Bem, D. J. (1988). Moving away from the world: life-course patterns of shy children. Developmental Psychology, 24, 824-833.

Castellanos, D., \& Hunter, T. (1999). Anxiety disorders in children and adolescents. Southern Medical Journal, 92, 946-953.

Central office of statistics (Centraal Bureau voor Statistiek, CBS). (2005). Retrieved 2005 from htpp://www.cbs.nl.

Chorpita, B. F., Taylor, A. A., Francis, S. E., Moffitt, C., \& Austin, A. A. (2004). Efficacy of modular cognitive behavior therapy for childhood anxiety disorders. Behavior Therapy, 35, 263-287.

Chorpita, B. F., Yim, L., Moffitt, C., Umemoto, L. A., \& Francis, S. F. (2000). Assessment of symptoms of DSM-IV anxiety and depression in children; A revised child anxiety and depression scale. Behaviour Research and Therapy, 38, 835-855.

Clark, L. A., \& Watson, D. (1991). Tripartite model of anxiety and depression: Psychometric evidence and taxonomic implications. Journal of Abnormal Psychology, 100, 316-336.

Cobham, V. E. (2003). Evaluation of a brief child-focused group-based intervention for anxietydisordered children. Behaviour Change, 20, 109-116.

Cobham, V. E., Dadds, M. R., \& Spence, S. H. (1998). The role of parental anxiety in the treatment of childhood anxiety. Journal of Consulting and Clinical Psychology, 66, 893-905.

Cornwall, E., Spence, S. H., \& Schotte, D. (1996). The effectiveness of emotive imagery in the treatment of darkness phobia in children. Behaviour Change, 13, 223-229.

Costello, E. J., Mustillo, S., Erkanli, A., Keeler, G., \& Angold, A. (2003). Prevalence and development of psychiatric disorders in childhood and adolescence. Archives of General Psychiatry, 60, 837-844. 
Craske, M. G. (1997). Fear and anxiety in children and adolescents. Bulletin of the Menninger Clinic, 61, A4-A36.

Crawford, A. M., \& Manassis, K. (2001). Familial predictors of treatment outcome in childhood anxiety disorders. Journal of the American Academy of Child and Adolescent Psychiatry, 40, 1182-1189.

Dadds, M. R., Heard, P. M., \& Rapee, R. M. (1992). The role of family intervention in the treatment of child anxiety disorders: Some preliminary findings. Behaviour Change, 9, 171-177.

Desgagné, A., Castilloux, A. M., Angers, J. F., \& LeLorier, J. (1998). The use of bootstrap statistical method for the pharmacoeconomic cost analysis of skewed data. Pharmacoeconomics, 13, 487-497.

DiNardo, P. A., Brown, T. A., \& Barlow, D. H. (1994). Anxiety Disorders Interview Schedule for DSM$I V$; lifetime version (ADIS-IV-L). San Antonio, TX: Psychological Corporation.

Dolan, P. (1997). Modelling valuations for EuroQol health states. Medical Care, 35, 1096-1108.

Drummond, M. F., O'Brien, B. J., Stoddart, G. L., \& Torrance, G. W. (1997). Methods for the Economic Evaluation of Health Care programmes. Oxford; Oxford Medical Publications.

Dupont, R. L., Rice, D. P., Miller, L. S., Shiraki, S. S., Rowland, C. R., \& Harwood, H. J. (1996). Economic costs of anxiety disorders. Anxiety, 2, 167-172.

Efron, B., \& Tibshirani, R. J. (1993). An introduction to the bootstrap. New York; Chapman and Hall.

Epkins, C. (1996). Cognitive specificity and affective confounding in social anxiety and dysphoria in children. Journal of Psychopathology and Behavioural Assessment, 18, 83-101.

EuroQol Group (1990). EuroQol- a new facility for the measurement of health related quality of life. Health Policy, 16, 199-208.

Fals-Stewart, W., Yates, B. T., \& Klostermann, K. (2005). Assessing the costs, benefits, cost-benefit ratio, and cost-effectiveness of marital and family treatments: Why we should and how we can. Journal of Family Psychology, 19, 28-39.

Flannery-Schroeder, E. C. \& Kendall, P. C. (2000). Group and individual cognitive-behavioral treatments for youth with anxiety disorders: A randomized clinical trial. Cognitive Therapy and Research, 24, 251-278.

Freer, C. B. (1980). Health diaries: a method of collecting health information. Journal of the Royal College of General Practitioners, 30, 279-282.

van der Gaag, R. J., Robbroeckx, L., Smid, G. A., \& Verhulst, F. C. (1996). Aan autisme verwante stoornissen. Kind en Adolescent, 17, 157-158.

Gallagher, H. M., Rabian, B. A., \& McCloskey, M. S. (2004). A brief group cognitive behavioural intervention for social phobia in childhood. Journal of Anxiety Disorders, 18, 459-479.

Ginsburg, G. S., \& Schlossberg, M. C. (2002). Family-based treatment of childhood anxiety disorders. International Review of Psychiatry, 14, 143-154.

Ginsburg, G. S., Silverman, W. K., \& Kurtines, W. K. (1995) Family involvement in treating children with phobic and anxiety disorders; A look ahead. Clinical Psychology Review, 15, 457-473.

Gold, M. R., Siegel, J. E., Russell, L. B., \& Weinstein, M. C. (1996). Cost-effectiveness in health and medicine. New York; Oxford University Press.

Goossens, M. E. J. B., Rutten-van Mölken, M. P. M. H., Vlaeyen, J. W. S., \& van der Linden, S. M. J. P. (2000). The cost diary: a method to measure direct and indirect costs in costeffectiveness research.. Journal of Clinical Epidemiology, 53, 688-695.

Greenberg, P. E., Sisitsky, T., Kessler, R. C., Finkelstein, S. N., Berndt, E. R., Davidson, J. R., et al. (1999). The economic burden of anxiety disorders in the 1990's. Journal of Clinical Psychiatry, 60, 427-435.

Hayward, C., Varady, S., Albano, A. M., Thienemann, M., Henderson, L., \& Schatzberg, A. F. (2000). Cognitive-behavioral group therapy for social phobia in female adolescents: Results of a pilot study. Journal of the American Academy of Child and Adolescent Psychiatry, 39, 721-726.

Hettema, J. M., Neale, M. C., \& Kendler, K. S. (2001). A review and meta-analysis of the genetic epidemiology of anxiety disorders. American Journal of Psychiatry, 158, 1568-1578. 
Heuzenroeder, L., Donnelly, M., Haby, M. M., Mihalopoulos, C., Rossell, R., Carter, R., et al. (2004). Cost-effectiveness of psychological and pharmacological interventions for generalised anxiety disorder and panic disorder. Australian and New Zealand Journal of Psychiatry, 38, 602-612.

Heyne, D., King, N. J., Tonge, B. J., Rollings, S., Young, D., Pritchard, M., et al. (2002). Evaluation of child therapy and caregiver training in the treatment of school refusal. Journal of the American Academy of Child and Adolescent Psychiatry, 41, 687-695.

Heyne, D., Sauter, F., Maric, M., Van Hout, R., Westenberg, M., Treffers, P., Van Widenfelt, B., \& Siebelink, B. (personal communication). Developmentally-tailored cognitive behavioural intervention for school refusal during adolescence.

Hien, D., Matzner, F. J., First, M. B., Spitzer, R. L., Gibbon, M., \& Williams, J. B. W. (1998). Structured Clinical Interview for DSM-IV-Child edition. New York: Columbia University.

Howard, B. L. \& Kendall, P. C. (1996). Cognitive-behavioral family therapy for anxiety-disordered children: A multiple-baseline evaluation. Cognitive Therapy and Research, 20, 423-443.

Hudson, J. L., Kendall, P. C., Coles, M. E., Robin, J. A., \& Webb, A. (2002). The other side of the coin: Using intervention research in child anxiety disorders to inform developmental psychopathology. Developmental Psychopathology, 14, 819-841.

In-Albon, T., \& Schneider, S. (in press). Psychotherapy of Childhood Anxiety Disorders: a MetaAnalysis. Psychotherapy and Psychosomatics.

Issakidis, C., Sanderson, K., Corry, J., Andrews, G., \& Lapsley, H. (2004). Modelling the population cost-effectiveness of current and evidence-based optimal treatment for anxiety disorders. Psychological Medicine, 34, 19-35.

Jacobs, P., \& Roos, N. (1990). Standard Cost Lists for Healthcare in Canada. Pharmacoeconomics, 15, 551-560.

James, A., Soler, A., \& Weatherall, R. (2005) Cognitive behavioural therapy for anxiety disorders in children and adolescents. The Cochrane Database of Systematic Reviews 2005, 4.

Järbrink, K., Fombonne, E., \& Knapp, M. (2003). Measuring the parental, service and cost impacts of children with autistic spectrum disorder: A pilot study. Journal of Autism and Developmental Disorders, 33, 395-402.

Jolly, J. B. (1993). A multi-method test of the cognitive content-specificity hypothesis in young adolescents. Journal of Anxiety Disorders, 7, 223-233.

Jolly, J. B., \& Dykman, R. A. (1994). Using self-report data to differentiate anxious and depressive symptoms in adolescents: Cognitive content specificity and global distress? Cognitive Therapy and Research, 18, 25-37.

Kane, M. T. \& Kendall, P. C. (1989). Anxiety disorders in children: A multiple-baseline evaluation of a cognitive-behavioral treatment. Behavior Therapy, 20, 499-508.

Kaslow, N. J., Stark, K. D., Printz, B., Livingston, R., \& Tsai, S. L. (1992). Cognitive Triad Inventory for Children: Development and relation to depression and anxiety. Journal of Clinical Child Psychology, 21, 339-347.

Kazdin, A. E., \& Nock, M. K. (2003). Delineating mechanisms of change in child and adolescent therapy: methodological issues and research recommendations. Journal of Child Psychology and Psychiatry and Allied Disciplines, 44, 1116-1129.

Keller, M. B., Lavori, P. W., Wunder, J., Beardslee, W. R., Schwarts, C. E., \& Roth, J. (1992). Chronic course of anxiety disorders in children and adolescents. Journal of the American Academy of Child and Adolescent Psychiatry, 31, 595-599.

Kendall, P. C. (1984). Behavioral assessment and methodology. In G. T. Wilson, C. M. Franks, K. D. Brownell and P. C. Kendall (Eds.). Annual review of behaviour therapy: Theory and practice (vol. 9, pp. 39-94). New York: Guilford Press.

Kendall, P. C. (1994). Treating anxiety disorders in children: results of a randomized clinical trial. Journal of Consulting and Clinical Psychology, 62, 100-110.

Kendall, P. C., Flannery-Schoeder, E., Panichelli-Mindel, S. M., Southam-Gerow, M., Henin, A., \& Warman, M. (1997). Therapy for youths with anxiety disorders: A second randomized clinical trial. Journal of Consulting and Clinical Psychology, 65, 366-380.

Kendall, P. C., Kane, M., Howard, B., \& Siqueland, L. (1990). Cognitive-behavioral therapy for anxious children: Treatment manual. Philadelphia: Department of Psychology, Temple University. 
Kendall, P. C., Safford, S., Flannery-Schroeder, E., \& Webb, A. (2004). Child anxiety treatment: Outcomes in adolescence and impact on substance use and depression at 7.4-year followup. Journal of Consulting and Clinical Psychology, 72, 276-287.

Kendall, P. C. \& Southam-Gerow, M. A. (1996). Long-term follow-up of a cognitive-behavioral therapy for anxiety-disordered youth. Journal of Consulting and Clinical Psychology, 64, 224230.

Kessler, R. C., McGonagle, K. A., Zhao, S., Nelson, C. B., Hughes, M., Eshleman, S., et al. (1994). Lifetime and 12-month prevalence of DSM-III-R psychiatric disorders in the United States. Archives of General Psychiatry, 51, 8-19.

King, N. J., Hamilton, D. I., \& Ollendick, T. H. (1988). Children's phobias: A behavioral perspective. New York: John Wiley

King, N. J., Tonge, B. J., Heyne, D., Pritchard, M., Rollings, S., Young, D., et al. (1998). Cognitivebehavioral treatment of school-refusing children: A controlled evaluation. Journal of the American Academy of Child and Adolescent Psychiatry, 37, 395-403.

King, N. J., Tonge, B. J., Turner, S., Heyne, D., Pritchard, M., Rollings, S., et al. (1999). Brief cognitive-behavioural treatment for anxiety-disordered children exhibiting school refusal. Clinical Psychology and Psychotherapy, 6, 39-45.

Klein, R. G., \& Pine, D. S. (2002). Anxiety Disorders. In Rutter, M. \& Taylor, E. (Eds.) Child and Adolescent Psychiatry, $4^{\text {th }}$ edition (pp. 486-510). Oxford, Blackwell Publishing.

Knapp, M. (1997). Economic evaluations and interventions for children and adolescents with mental health problems. Journal of Child Psychology and Psychiatry, 38, 3-25.

Knapp, M., Scott, S., \& Davies, J. (1999). The cost of antisocial behaviour in younger children. Clinical Child Psychology and Psychiatry, 4, 457-473.

Kovacs, M. \& Devlin, B. (1998). Internalizing disorders in childhood. Journal of Child Psychology and Psychiatry and Allied Disciplines, 39, 47-63.

Kraemer, H. C., Wilson, G. T., Fairburn, C. G., \& Agras, W. S. (2002). Mediators and moderators of treatment effects in randomized clinical trials. Archives of General Psychiatry, 59, 877-883.

Last, C. G., Hansen, C., \& Franco, N. (1998). Cognitive-behavioral treatment of school phobia. Journal of the American Academy of Child and Adolescent Psychiatry, 37, 404-411.

Last, C. G., Perrin, S., Hersen, M., \& Kazdin, A. E. (1992). DSM-III-R anxiety disorders in children: sociodemographic and clinical characteristics. Journal of the American Academy of Child and Adolescent Psychiatry, 31, 1070-1076.

Leaf, P. J., Alergia, M., Cohen, P., Goodman, S. H., Horwitz, S. M., Hoven, C. W., et al. (1996). Mental health service use in the community and schools: Results from the fourcommunity MECA study. Child and Adolescent Psychiatry, 35, 889-897.

Leon, A. C., Portera, L., \& Weissman, M. M. (1995). The social costs of anxiety disorders. British Journal of Psychiatry, 27, 19-22.

Manassis, K., Avery, D., Butalia, S., \& Mendlowitz, S. (2004). Cognitive-behavioral therapy with childhood anxiety disorders: functioning in adolescence. Depression and Anxiety, 19, 209216.

Manassis, K., Mendlowitz, S. L., Scapillato, D., Avery, D., Fiksenbaum, L., Freire, M., et al. (2002). Group and individual cognitive-behavioral therapy for childhood anxiety disorders: A randomised trial. Journal of the American Academy of Child and Adolescent Psychiatry, 41, 1423-1430.

Manca, A., Hawkins, N., \& Sculpher, M. J. (2005). Estimating mean QALYs in trial-based costeffectiveness analysis: the importance of controlling for baseline utility. Health Economics, 14, 487-496.

Marciniak, M., Lage, M. J., Landbloom, R. P., Dunayevich, E., \& Bowman, L. (2004). Medical and productivity costs of anxiety disorders: case control study. Depression and Anxiety, 19, 112120.

Marien, W. E., \& Bell, D. J. (2004). Anxiety- and depression-related thoughts in children: Development and evaluation of a cognition measure. Journal of Clinical Child and Adolescent Psychology, 33, 717-730.

Marra, C. A., Woolcott, J. C., Kopec, J. A., Shojania, K., Offer, R., Brazier, J. E., et al. (2005). A comparison of generic, indirect utility measures (the HUI2, HUI3, SF-6D and the EQ-5D) 
and disease-specific instruments (the RAQol and the HAQ) in rheumatoid arthritis. Social Science and Medicine, 60, 1571-1582.

McCrone, P., Knapp, M., Proudfoot, J., Ryden, C., Cavanagh, K., Shapiro, D. A., et al. (2004). Costeffectiveness of computerised cognitive behavioural therapy for anxiety and depression in primary care: randomised control trial. British Journal of Psychiatry, 185, 55-62.

Meltzer-Brody, S., \& Davidson, J. R. T. (2000). Completeness of response and quality of life in mood and anxiety disorders. Depression and Anxiety, 12, supplement 1, 95-101.

Mendlowicz, M. V., \& Stein, M. B. (2000). Quality of life in individuals with anxiety disorders. American Journal of Psychiatry, 157, 669-682.

Mendlowitz, S. L., Manassis, K., Bradley, S., Scapillato, D., Miezitis, S., \& Shaw, B. F. (1999). Cognitive-behavioral group treatments in childhood anxiety disorders: The role of parental involvement. Journal of the American Academy of Child and Adolescent Psychiatry, $38,1223-1229$.

Menzies, R. G., \& Clarke J. C. (1993). A comparison of in vivo and vicarious exposure in the treatment of childhood water phobia. Behaviour Research and Therapy, 31, 9-15.

Mifsud, C., \& Rapee, R. M. (2005). Early intervention for childhood anxiety in a school setting: Outcomes for an economically disadvantaged population. Journal of the American Academy Of Child And Adolescent Psychiatry, 44, 996-1004.

Muris, P., Dreessen, L., Bögels, S., Weckx, M., \& van Melick, M. (2003). A questionnaire for screening a broad range of DSM-defined anxiety disorder symptoms in clinically referred children and adolescents. Journal of Child Psychology and Psychiatry, 44, 1-8.

Muris, P., Mayer, B., Bartelds, E., Tierney, S., \& Bogie, N. (2001). The revised version of the Screen for Child Anxiety Related Emotional Disorders (SCARED-R): Treatment sensitivity in an early intervention trial for childhood anxiety disorders. British Journal of Clinical Psychology, 40, 323-336.

Muris, P., Meesters, C., \& van Melick, M. (2002). Treatment of childhood anxiety disorders; A preliminary comparison between cognitive-behavioral group therapy and a psychological placebo intervention. Journal of Behavior Therapy and Experimental Psychiatry, 33, 143-158.

Muris, P., Merkelbach, H., van Brakel, A., \& Mayer, B. (1999b). The Screen for Child Anxiety Related Emotional Disorders (SCARED): Further evidence for its reliability and validity. Anxiety Stress and Coping, 12, 411-425.

Muris, P., Merckelbach, H., Gadet, B., Mouleart, V., \& Tierney, S. (1999c). Sensitivity for treatment effects of the Screen for Child Anxiety Related Emotional Disorders. Journal of Psychopathology and Behavioral Assessment, 21, 323-335

Muris, P., Merckelbach, H., Holdrinet, I., \& Sijsenaar, M. (1998). Treating phobic children: Effects of EMDR versus exposure. Journal of Consulting and Clinical Psychology, 66, 193-198.

Muris, P., Merckelbach, H., Kindt, M., Bögels, S., Dreessen, L., van Dorp, C., et al. (2001). The utility of Screen for Child Anxiety Related Emotional Disorders (SCARED) as a tool for identifying children at high risk for prevalent anxiety disorders. Anxiety Stress and Coping, 14, 265-283.

Muris, P., Merckelbach, H., Mayer, B., van Brakel, A., Thissen, S., Moulaert, V., et al. (1998). The Screen for Child Anxiety Related Emotional Disorders and traditional childhood anxiety measures. Journal of Behavior Therapy and Experimental Psychiatry, 29, 327-339.

Muris, P., Merkelbach, H., Mayer, B., \& Prins, E. (2000). How serious are common childhood fears? Behaviour Research and Therapy, 38, 217-228.

Muris, P., Merkelbach, H., Schmidt, H., \& Mayer, B. (1999a). The revised version of the Screen for Child Anxiety Related Emotional Disorders (SCARED-R): Factor structure in nonclinical children. Personality and Individual Differences, 26, 99-112.

Muris, P., \& Steerneman, P. (2001). The revised version of the Screen for Child Anxiety Related Emotional Disorders (SCARED-R): First evidence for its reliability and validity in a clinical sample. British Journal of Clinical Psychology, 40, 35-44.

National Institute of Budget information (Nationaal Instituut voor Budgetvoorlichting, NIBUD) (2005). Retrieved 2005 from http://www.nibud.nl.

National Institute of Mental Health (1992), Diagnostic Interview Schedule for Children (DISC), Version 2.3. New York: New York State Psychiatric Institute. 
Nauta, M. H., Scholing, A., Emmelkamp, P. M. G., \& Minderaa, R. B. (2001). Cognitive-behavioural therapy for anxiety disordered children in a clinical setting: Does additional cognitive parent training enhance treatment effectiveness? Clinical Psychology and Psychotherapy, 8 , 330-340.

Nauta, M. H., Scholing, A., Emmelkamp, P. M. G., \& Minderaa, R. B. (2003). Cognitive-behavioural therapy for anxiety disordered children in a clinical setting: No additional effect of a cognitive parent training. Journal of the American Academy of Child and Adolescent Psychiatry, 42, 1270-1278.

Newman, D. L., Moffitt, T. E., Caspi, A., Magdol, L., Silva, P. A., \& Stanton, W. R. (1996). Psychiatric disorder in a birth cohort of young adults: Prevalence, comorbidity, clinical significance, and new case incidence from ages 11-21. Journal of Consulting and Clinical Psychology, 64, 552-562.

Ollendick, T. H., Yule, W., \& Ollier, K. (1991). Fears in British children and their relationship to manifest anxiety and depression. Journal of Child Psychology and Psychiatry, 32, 321-331.

Oostenbrink, J. B., Koopmanschap, M. A., \& Rutten, F. F. H. (2005). Handleiding voor kostenonderzoek: Methoden en richtlijnprijzen voor economische evaluaties in de gezondheidszorg. Amstelveen; College voor zorgverzekeringen.

Ost, L. G., Svensson, L., Hellström, K., \& Lindwall, R. (2001). One-session treatment of specific phobias in youths: A randomized clinical trial. Journal of Consulting and Clinical Psychology, 69, 814-824.

Piaget. J. (1987). Possibility and necessity, Vol. 1. The role of possibility in cognitive development. Minneapolis: University of Minnesota Press.

Pierce, K. A., \& Kirkpatrick, D. R. (1992). Do men lie on fear surveys? Behaviour Research and Therapy, $30,415-418$.

Polder, J. J., Takken, J., Meerding, W. J., Kommer, G. J., \& Stokx, L. J. (2002). Kosten van ziekten in Nederland. Retrieved 2002, from http://www.rivm.nl/kostenvanziekten.

Prins, P. J. M. (2002). De dynamiek van cognitie en emotie in de ontwikkeling van angststoornissen bij kinderen. Kind en Adolescent, 23, 250-265.

Prins, P. J. M. \& de Haan, E. (contact persons, personal communication). A multi center study to investigate mediators and moderators of effective Cognitive Behavioural Therapy in children with anxiety disorders. Participating centres; Clinical child and youth psychology, University of Amsterdam, de Bascule Amsterdam and Accare Child and youth psychiatry Groningen.

Ramsey, S., Willke, R., Briggs, A., Brown, R., Buxton, M., Chawla, A., et al. (2005). Good research practices for cost-effectiveness analysis alongside clinical trials: The ISPOR RCT-CEA task force report. Value in Health, 5, 521-533.

Rapee, R. M., Spence, S., Cobham, C., \& Wignall, A. (2000). Helping your anxious child: A step-bystep guide for parents. Oakland, CA: New Harbinger.

Rice, D. P., \& Miller, L. S. (1995). Health economies and cost implications of anxiety and other mental disorders in the US. British Journal of Psychiatry, 173, 4-9.

Ronan, K. R. \& Kendall, P. C. (1997). Self-talk in distressed youth: States-of-mind and content specificity. Journal of Clinical Child Psychology, 26, 330-337.

Ronan, K. R., Kendall, P. C., \& Rowe, M. (1994). Negative affectivity in children: Development and validation of a self-statement questionnaire. Cognitive Therapy and Research, 18, 509-528.

Ronan, K. R., Rowe, P., \& Kendall, P. C. (November, 1988). Children's anxious self-statement questionnaire (CASSQ); Development and validation. Paper presented at the Association for the Advancement of Behavior Therapy Convention, New York.

Rosenberg, F. R., \& Simmons, R. G. (1975). Sex differences in the self-concept in adolescence. Sex roles, 1, 147-159.

Schniering, C. A., \& Rapee, R. M. (2002). Development and validation of a measure of children's automatic thoughts: The Children's Automatic Thoughts Scale. Behaviour Research and Therapy, 40, 1091-1109.

Schniering, C. A., \& Rapee, R. M. (2004a). The relationship between automatic thoughts ands negative emotions in children and adolescents: A test of the cognitive content-specificity hypothesis. Journal of Abnormal Psychology, 113, 464-470. 
Schniering, C. A., \& Rapee, R. M. (2004 $\left.{ }^{\mathrm{b}}\right)$. The structure of negative self-statements in children and adolescents: A confirmatory Factor-analytic approach. Journal of Abnormal Child Psychology, 32, 95-109.

Sculpher, M. J., Claxton, K., Drummond, M., Mcabe, C. (2006). Whither trial-based economic evaluation for health care decision making? Health Economics, 15, 677-687.

Shortt, A. L., Barrett, P. M., \& Fox, T. L. (2001). Evaluating the FRIENDS program: A cognitivebehavioral group treatment for anxious children and their parents. Journal of Consulting and Clinical Psychology, 30, 525-535.

Silverman, W. K., \& Eisen, A. R. (1992). Age differences in the reliability of parent and child reports of child anxious symptomatology using a structures interview. Journal of the American Academy of Child and Adolescent Psychiatry, 31, 117-124.

Silverman, W. K., Kurtines, W. M., Ginsburg, G. S., Weems, C. F., Lumpkin, P. W., \& Carmichael, D. H. (1999). Treating anxiety disorders in children with group cognitive-behavioral therapy: A randomized clinical trial. Journal of Consulting and Clinical Psychology, 67, 995-1003.

Silverman, W. K., Kurtines, W. M., Weems, C. F., Ginsburg, G. S., Rabian, B., \& Serafini, L. T. (1999). Contingency management, self-control, and educational support in the treatment of childhood phobic disorders: a randomized clinical trial. Journal of Consulting and Clinical Psychology, 67, 675-687.

Silverman, W. K., \& Nelles, W. B. (1988). The Anxiety Disorders Interview Schedule for children. Journal of the American Academy of Child and Adolescent Psychiatry, 27, 772-778.

Simon, E., Bögels, S. M., \& Dirksen, C.D. (personal communication). Prevention of childhood anxiety disorders: A comparison of the effects of child cognitive-behaviour intervention, parental cognitive-behaviour intervention, and no intervention in a Dutch community sample of at-risk children.

Siqueland, L., \& Diamond, G. S. (1998). Engaging parents in cognitive behavioural treatment for children with anxiety disorders. Cognitive and Behavioral Practice, 5, 81-102.

Siqueland, L., Rynn, M., \& Diamond, G. S. (2005). Cognitive behavioural and attachment based family therapy for anxious adolescents: Phase I and II studies. Journal of Anxiety Disorder, 19, 361-381.

Sleed, M., Eccleston, C., Beecham, J., Knapp, M., \& Jordan, A. (2005). The economic impact of chronic pain in adolescence: Methodological considerations and preliminary costs-ofillness study. Pain, 119, 183-190.

Southam-Gerow, M. A., Kendall, P. C., \& Weersing, V. R. (2001). Examining outcome variability: Correlates of treatment response in a child and adolescent anxiety clinic. Journal of Clinical Child Psychology, 30, 422-436.

Spence, S. H. (1998). A measure of anxiety symptoms among children. Behaviour Research and Therapy, 36, 545-566.

Spence, S. H., Donovan, C., \& Brechman-Toussaint, M. (1999). Social skills, social outcomes, and cognitive features of childhood social phobia. Journal of Abnormal Psychology, 108, 211-221.

Spence, S. H., Donovan, C., \& Brechman-Toussaint, M. (2000). The treatment of childhood social phobia: The effectiveness of a social skills training-based, cognitive-behavioural intervention, with and without parental involvement. Journal of Child Psychology and Psychiatry, 41, 713-726.

Spielberger, C. (1973). Manual for State-Trait Anxiety Inventory for Children. Palo Alto, CA: Consulting Psychologists Press.

Steinglass, P. (1996). Family therapy's future. Family Process, 35, 403-405.

Stolk, E. A., Busschbach, J. J. V., \& Vogels, T. (2000). Performance of the EuroQol in children with imperforate anus. Quality of Life Research, 9, 29-38.

Strauss, C. C., Frame, C. L., \& Forehand, R. (1987). Psychosocial impairment associated with anxiety in children. Journal of Clinical Child Psychology, 16, 235-239.

Strauss, C. C., \& Last, C. G. (1993). Social and simple phobias in children. Journal of Anxiety Disorders, 7, 141-152.

Strauss, C. C., Last, C. G., Hersen, M., \& Kazdin, A. E. (1988). Association between anxiety and depression in children and adolescents with anxiety disorders. Journal of Abnormal Child Psychology, 16, 57-68. 
Sytema, S., \& Koopmans, P. (1998). Psychische stoornissen in de volwassen bevolking; een verkenning van omvang, gevolgen en kosten. Den Haag, SDU uitgevers.

Task Force on Promotion and Dissemination of Psychological Procedures, Division of Clinical Psychology (1995). Training in and dissemination of empirically-validated psychological treatments: report and recommendations. Clinical Psychologist, 48, 3-23.

Thienemann, M., Moore, P, \& Tompkins, K. (2006). A parent-only group intervention for children with anxiety disorders: Pilot study. Journal of the American Academy of Child and Adolescent Psychiatry, 45, 37-46.

Toren, P., Wolmer, L., Rosental, B., Eldar, S., Koren, S., Lask, M., et al. (2000). Case series: Brief parent-child group therapy for childhood anxiety disorders using a manual-based cognitive-behavioral technique. Journal of the American Academy of Child and Adolescent Psychiatry, 39, 1309-1312.

Treffers, P. D. A., .Ferdinand, R. F., Utens, E. M. W. J., van Widenfelt, B. M., Liber, J. M., \& van der Leeden, A. J. M. (personal communication). Cognitive behavioral treatment of childhood anxiety disorders: patient characteristics, treatment format and treatment outcome.

Tudor Edwards, R., \& Thalanany, M. (2001). Trade-offs in the conduct of economic evaluations of child mental health services. Mental Health Services Research, 3, 99-105.

Verhulst, F. C., \& van der Ende, J. (1997). Factors associated with child mental health service use in the community. Journal of the American Academy for Child and Adolescent Psychiatry, 36, 901909.

Verhulst, F. C., van der Ende, J., Ferdinand, R. F., \& Kasius, M. C. (1997). The prevalence of DSM-III$\mathrm{R}$ diagnoses in a national sample of Dutch adolescents. Archives of General Psychiatry, 54, 329-336.

Verhulst, F. C., van der Ende, J., \& Koot, H. M. (1996). Handleiding voor de CBCL (4 - 18). Rotterdam: Erasmus Universiteit Rotterdam/Academisch Ziekenhuis Rotterdam, afdeling Kinder- en Jeugdspychiatrie, Sophia Kinderziekenhuis.

Wallander, J. L., Schmitt, M., \& Koot, H. M. (2001). Quality of life measurement in children and adolescents: Issues, instruments, and applications. Journal of Clinical Psychology, 57, 571585.

Weems, C. F., Berman, S. L., Silverman, W. K., \& Saavedra, L. M. (2001). Cognitive errors in youth with anxiety disorders: The linkages between negative cognitive errors and anxious symptoms. Cognitive Therapy and Research, 25, 559-575.

Weiss, B., Catron, T., Harris, V., \& Phung, T. M. (1999). The effectiveness of traditional child psychotherapy. Journal of Consulting and Clinical Psychology, 67, 82-94.

Weisz, J. R., Weiss, B., Han, S. S., Granger, D. A., \& Morton, T. (1995). Effects of psychotherapy with children and adolescents revisited: A meta analysis of treatment outcome studies. Psychological Bulletin, 117, 450-468.

Westenberg, P. M., Drewes, M. J., Goedhart, A. W., Siebelink, B. M., \& Treffers, P. D. A. (2004). A developmental analysis of self-reported fears in late childhood through mid adolescence: social-evaluative fears on the rise? Journal of Child Psychology and Psychiatry, 45, 481-495.

Windheuser, H. J. (1977). Anxious mothers as models for coping with anxiety. Behavioural Analysis and Modification, 2, 39-58.

de Wit, C. A. M. (2000). Depressie bij kinderen en adolescenten; Theorie en onderzoek, diagnostiek en behandeling. Bohn Stafleu van Loghum, Houten/Diegem.

de Wit, G. A., Busschbach, J. J. V., \& De Charro, F. T. H. (2000). Sensitivity and perspective in the valuation of health status: whose values count? Health Economics, 9, 109-126.

Wood, J. J., Piacentini, J. C., Southam-Gerow, M., Chu, B. C., \& Sigman, M. (2006). Family cognitive behavioural therapy for child anxiety disorders. American Academy of Child and Adolescent Psychiatry, 45, 314-321.

World Health Organization (WHO). International Classification of Diseases, injuries and causes of death, $9^{\text {th }}$ revision. Geneve, 1977.

Zatz, S., \& Chassin, L. (1983). Cognitions of test-anxious children. Journal of Consulting and Clinical Psychology, 51, 526-534. 


\section{Samenvatting}


Tussen januari 2002 en december 2005 is er een grootschalig onderzoek uitgevoerd naar de kosten-effectiviteit van gezins cognitieve gedragstherapie en individuele of kind cognitieve gedragstherapie bij gezinnen met een klinisch angstig kind. Dit houdt in dat er gekeken werd welke van de 2 behandelingen (individuele of gezins cognitieve gedragstherapie) beter werkt (effectiever is) en welke therapie, relatief gezien, het goedkoopst is in het behandelen van klinisch angstige kinderen. Klinisch angstige kinderen zijn kinderen die zo angstig zijn dat hun leven erdoor beïnvloed wordt en een behandeling nodig hebben. Cognitieve gedragstherapie is een behandeling waarbij angstige gedachten veranderd worden in minder angstige gedachten. Ook leert het kind dat het angstige situaties of dingen die het vermijdt juist moet gaan opzoeken en zo gaat wennen aan de angst totdat de angst weg is. In totaal hebben 128 gezinnen meegedaan aan dit onderzoek die gelijkmatig werden verdeeld over de individuele en de gezins cognitieve gedragstherapie.

Naast kosten-effectiviteit is ook onderzocht hoeveel geld gezinnen met een klinisch angstig kind, de maatschappij kosten. Daarbij zijn kosten van de gezondheidszorg en kosten buiten de gezondheidszorg zoals opvang gemeten. Bovendien zijn indirecte kosten, zoals kosten die ouders maken als ze niet naar hun werk kunnen omdat ze bij het angstig kind moeten blijven, en eigen gemaakte kosten, zoals reisgeld, in kaart gebracht. Deze kosten zijn door de gezinnen opgeschreven in een kostendagboek. Opgeteld komen de totale kosten voor alle gezinnen in Nederland met een klinisch angstig kind uit op een bedrag van 20.3 miljoen euro. Deze kosten zijn bijna 21 keer zoveel als de kosten van gezinnen uit de algemene bevolking zonder een angstig kind. Naast gezondheidzorgkosten, zijn de kosten voor ouders die niet naar hun werk kunnen door de angsten van het kind en schoolverzuim van het angstig kind, het hoogst. Deze kosten zouden misschien bespaard kunnen worden als angstige kinderen een goede behandeling krijgen.

Om te kijken of de behandeling goed werkt, zijn voor en na behandeling interviews afgenomen bij de gezinnen om te bepalen of er een angststoornis aanwezig was. Ook zijn er een aantal nieuwe of vertaalde vragenlijsten afgenomen zoals de CATS die mogelijke veranderingen in negatieve gedachten bij kinderen meet voor en na therapie. De CATS is in het Nederlands vertaald. Een andere vragenlijst, de SCARED meet angstklachten bij kinderen. De oorspronkelijke SCARED is uitgebreid met 5 sociale angst vragen zodat sociale angst beter in beeld gebracht kon worden. Door de uitkomsten op deze vragenlijsten te vergelijken met kinderen uit de algemene bevolking zonder angsten, kunnen zogenaamde afkappunten bepaald worden. Deze afkappunten kunnen in de behandelstudie gebruikt worden om te kijken of klinisch angstige kinderen onder het afkappunt zitten en dus ongeveer dezelfde hoeveelheid angstklachten en negatieve gedachten hebben als andere kinderen.

In de behandelstudie is aangetoond dat individuele cognitieve gedragstherapie beter werkt dan gezins cognitieve gedragstherapie. Dit wil 
zeggen dat er meer kinderen vrij van hun angststoornis waren na de individuele behandeling dan na de gezins behandeling. Ook uit de literatuur kan geconcludeerd worden dat het betrekken van ouders bij de behandeling van het angstig kind niet beter werkt dan wanneer het kind alleen behandeld wordt. Bovendien is gevonden dat cognitieve gedragstherapie beter werkt bij 8 tot 12 jarige angstige kinderen dan bij 13 tot 17 jarigen. Verder laten de resultaten zien dat cognitieve gedragstherapie minder goed werkt bij angstige kinderen van wie de ouders zelf ook angstig zijn.

Uiteindelijk is de kosten-effectiviteit studie uitgevoerd. Dit is de eerste studie ter wereld die gekeken heeft naar de kosten van cognitieve gedragstherapie bij angstige kinderen. Resultaten tonen aan dat individuele cognitieve gedragstherapie beter werkt en goedkoper is dan gezins cognitieve gedragstherapie.

De conclusie is dat individuele cognitieve gedragstherapie de beste keus is om klinisch angstige kinderen te behandelen omdat het beter werkt en goedkoper is. Nieuwe onderzoeken moeten laten zien of de gevonden resultaten ook gelden op langere termijn bijvoorbeeld over een paar jaar. Ook zal er onderzocht moeten worden welk gedeelte van de cognitieve gedragstherapie het best werkt en waarom. Bovendien zou gekeken moeten worden of het niet beter en goedkoper is om kinderen, die een verhoogd risico hebben om angstig te worden, preventief te behandelen dus voordat ze ernstige angstklachten krijgen. Dan kan bepaald worden of het beter en goedkoper is om vroegtijdig kinderen met angstklachten te behandelen of dat ze pas behandeld moeten worden als ze een ernstige angststoornis hebben. 
Abstract 
Between January 2002 and December 2005, a randomized control trial was conducted to investigate the cost-effectiveness of family Cognitive Behavioural Therapy (CBT) versus individual CBT. In total 128 families were randomized to individual CBT $(n=64)$ or family CBT $(n=64)$. Before investigating the costeffectiveness, the costs of families with clinically anxious children referred for treatment were examined, which was never been done before. The Dutch societal costs of illness in clinically anxious children and their families, using the bottom up approach by means of a prospective cost diary amounted to approximately 20.3 million euros, which is almost 21 times as high compared to families from the general population. Besides, health care costs, productivity loss of the parents and school absence of the child contributed mostly to the total costs. These high costs can be saved by providing an effective treatment to families with clinically anxious children.

Effectiveness was measured by means of a semi-structures interview to obtain diagnostic status and with several newly developed or translated questionnaires including the Child Automatic Thoughts Scale (CATS; Schniering \& Rapee, 2002) and the Screen for Child Anxiety Related Emotional Disorders (Muris et al., 1999). The CATS was translated into a Dutch version to measure change in cognition, which is sporadically assed in previous effectiveness studies. The existing 66-item SCARED did not sufficiently measure social phobia, therefore this questionnaire measuring anxiety symptoms was expanded by adding 5 extra social phobia items. Cut-off scores for these two questionnaires were obtained by differentiating clinically anxious children from children from the general population. These cut-off scores are important in the effectiveness study to determine whether a child reached a normal level of anxiety symptoms and negative automatic thoughts after treatment.

In contrast to expectations, the effectiveness study showed that individual CBT was more effective than family CBT. Also when reviewing the literature on parental involvement, the evidence is growing that parental involvement has no additive value. Also contrary to our hypothesis, family CBT was not more effective in young children. A more general effect was found namely; CBT is more effective in 8- to 12-year-old children compared to 13- to 17-year-old adolescents. Again, in contrast to the hypothesis, family CBT was not more effective when parents suffered from anxiety disorders themselves. The more general finding that CBT is less effective if parents suffer from anxiety disorders themselves was found.

Finally, the cost-effectiveness study was conducted. Previous studies on CBT in anxious children only investigated effectiveness. This was the first study also taken into account the costs of CBT in anxious children. Results show that although costs and effects did not significantly differ between individual CBT and family CBT, cost-effectiveness ratios, expressing incremental cost per anxiety-free child and per QALY, indicated that individual CBT was more costeffective than family CBT. 
In conclusion, individual CBT seems the best choice for treating children with clinical anxiety disorders, in terms of effectiveness and costeffectiveness. Future studies should investigate moderators and mediators, mechanisms of change of CBT, the long-term term cost-effectiveness, costeffectiveness in children with anxiety disorders in general and costeffectiveness in prevention of anxiety disorders to enhance the effectiveness of CBT for children with anxiety disorders, to reduce the costs associated with anxiety disorders in clinically referred children and as a result to obtain optimum cost-effectiveness. 


\section{Dankwoord}

Het dankwoord van een proefschrift is het meest gelezen stukje van een proefschrift. Zeker bij een multi center studie zoals beschreven in dit proefschrift, kom je er niet omheen. Dit proefschrift is dan ook tot stand gekomen dankzij de hulp en medewerking van veel mensen. Zonder hen zou dit proefschrift niet in deze vorm voor u liggen.

Allereerst, mijn promotor, Susan Bögels. Bedankt voor jouw begeleiding tijdens mijn promotie traject. De afgelopen jaren heb ik veel bewondering gekregen voor je gedrevenheid, kennis, onderzoeksvaardigheden en therapeutische inzet bij kinderen met psychopathologie. Weinig mensen kunnen wetenschappelijke kennis en de klinische praktijk zo goed combineren als jij. Ook heb ik veel van je geleerd over artikelen schrijven, dankzij je zorgvuldige en grondige feedback op de artikelen-in-wording. Verder weet jij als geen ander mensen te motiveren om het beste uit henzelf te halen. Bedankt voor je vertrouwen in mij bij het uitvoeren en afronden van de mc-studie.

Mijn co-promotor, Carmen, je wordt bedankt! En nu serieus: heel erg bedankt voor de fijne samenwerking en de economische kennis die je op mij hebt overgebracht. Ik heb de kosten analyses heel lang voor me uitgeschoven maar jij hebt met je enthousiasme voor deze tak van wetenschap mij hiervoor weten te motiveren. Jij maakte van een economie-inzichtloos iemand, een MTA-er, daar heb je veel energie ingestoken en daar ben ik je heel dankbaar voor. De materie was niet altijd makkelijk maar jouw positieve kijk op dingen sleepte mij door de kostenanalyses heen. En zie hier het resultaat; een half boekje met economische evaluaties! Hopelijk kunnen we in de toekomst nog samenwerken (dat EuroQol artikel?)!

De Beoordelingscommissie, Arnoud Arntz, Caroline Braet, Anita Jansen, Hans Severens, bedankt voor het beoordelen van het manuscript.

De Paranimphen Manon en Lotte, ik ben ontzettend blij dat jullie mij op de "dag des oordeels" willen bijstaan. Manon, ik weet dat jij mijn therapeut zal zijn als ik dan helemaal gestresst ben en Lotte, jij zal alles voor mij relativeren met je humor. Door de jaren heen zijn jullie meer geworden dan alleen collega's. Als ik weer eens een onderzoeksdipje had dan kon ik bij jullie terecht, dank voor jullie steun en gezelligheid!

Alle medewerkers van de Mc-studie van Accare, De Bascule, Mondriaanzorggroep Maastricht; GGZ Groningen zuid, GGZ Eindhoven en GGZ's Hertogenbosch/Herlaarhof. 
Alle coördinatoren, Maaike Nauta, Else de Haan, Jaap Ringrose, Dries Brinkman, Carla Appelboom, en Karen Appelboom, bedankt voor jullie inzet, input en het regelen van de studie binnen de centra Accare, de Bascule, GGZ Eindhoven, GGZ Groningen, Mondriaanzorggroep Maastricht, en Herlaarhof. Een speciaal dank aan Maaike; je hebt me de eerste maanden wegwijs gemaakt in de onderzoekswereld en me geholpen om alles op de rails te zetten!

Alle therapeuten, Peter van de Boomen, Marieken Braber, Marjolien Bus, Pamela Clifford, Sebina Dijken, Ilse van Doorn, Cora van Dyk, Nicole van Gastel, Carolien Gevers, Leonie van Ginkel, Karin Hermeler, Hanneke van Kasteren, Adraina Kelling, Ronnke Mud, Janet Noorloos, Charlotte Nuhoff, Bertien Paanakker, Mirjam Pijpers, Ardy Quaedackers, Cornelie Samson, Johan van Schaik, Anke Schooten, Mirjam Smid, Maria van de Ven, Linda Verhaak, Leonieke Vet, Sjoukje Warners, Jiska Weijermans, Renske Wichers, Eugene Wijnands, en Carola de Wit, jullie ook bedankt voor jullie inzet, de uitgevoerde behandelingen en de gezellige terugkomdagen.

Alle onderzoeksassistenten, Lotte Bamelis, Nienke Blom, Corine van der Bruggen, Mirten Halverhout, Sandra van der Hoek, Loes Leushuijs, Marion van Melick, Suzanne Mol, Harma Moorlag, Suzanne Pielage, Sarah de Schutter, Saskia Straus, Ingeborg Visser, Miryam Weckx, Jenneke Wiersma, Lidewij Wolters, en Manon Zeijl, bedankt voor jullie inzet, het afnemen van de ellenlange ADISsen en de vragenlijsten en de leuke e-mail correspondentie heen en weer. Speciaal dank aan Harma en Sandra aan de ander kant van het land, naast het werk zijn we ook privé elkaar blijven zien en mailen, dank voor jullie luisterend oor, humor en gezelligheid.

En natuurlijk alle kinderen, ouders, broertjes en zusjes voor deelname aan het onderzoek en het doorstaan van de interviews en metingen.

Dank aan Peter Muris; ik weet niet of ik zonder jou de onderzoekswereld zou zijn ingestapt. Jij zette me op de juiste weg met mijn afstudeeronderzoek. Ik mag hopen dat ik ooit zoveel op papier krijg als jij!

Corien Bögels, de omslag van mijn proefschrift is nog beter dan ik ooit bedacht had, bedankt voor je creatieve illustraties!

Alle DMKEP-ers ; bedankt voor de gezellige 4,5 jaar binnen de vakgroep met leuke dagjes-uit, kerstdiners, congressen en andere bijeenkomsten en vooral de praatjes in de keuken! Alle AIO's door de jaren heen voor hun wijze raad wat betreft onderzoek doen, Marisol voor de helpende hand aan het einde van mijn proefschrift, Jeanine voor de gezellige onderonsjes op kamer 1330, Pomme voor de leuke tijd ook op kamer 1330, Corine, Yvonne, Suzanne Mol en Wonneke. 
Het secretariaat en dan vooral Carla en Emmy, voor het helpen te regelen van allerlei administratieve rompslomp die komt kijken bij het organiseren van een multi-center studie. Emmy bedankt voor het muggenziften! Erik bedankt voor al je statistische hulp! Alle angstige AIO's bedankt voor de leuke en leerzame bijeenkomsten!

Mijn familie, pap, mam, Kim, Eddy, Dahnee, en schoonfamilie, Gonny, Aad, Bart, Katja, Marijn en Mayke en andere familieleden, dank voor jullie steun tijdens het promotieproces. Mijn vrienden, vooral Lijuen, Neeltje, Wilbert, Hans, en Juliette. Alle medewerkers van de Kinderboerderij de Wieringenberg bedankt voor de ontspanning in de weekenden.

Bob, mijn grootste steunpilaar tijdens het hele onderzoeksgebeuren. Tijdens het begin van mijn promotie onderzoek hebben we elkaar (weer) gevonden en niet meer los gelaten. Je steunt mij in al mijn carrière beslissingen en bent er zelfs voor naar Maastricht gekomen. De dalen en de pieken van het onderzoek, je hebt het allemaal van heel dichtbij meegemaakt, bedankt voor je geduld, je luisterend oor, je relativerend vermogen, en bovenal je liefde! 


\section{Curriculum Vitae}

Denise Bodden was born on the 30th of June in 1978, in Maastricht, The Netherlands, as daughter of Jan Bodden en Els Musson and little sister of Kim. She followed secondary school (HAVO and VWO) at the Jeanne D'arc college in Maastricht. From 1997 to 2001 she studied Psychology at the University of Maastricht. Her $3^{\text {rd }}$ year field of study was Neuropsychology and $4^{\text {th }}$ year field of study was psychopathology. Her research master was supervised by Peter Muris. From 2002 to 2006, Denise worked as a researcher at the Department of Medical, Clinical and Experimental Psychopathology at the University of Maastricht and at the department of Medical Psychology at the Academic Hospital in Maastricht. Her research topic was to investigate the costeffectiveness of family versus individual CBT in children with anxiety disorders of which the results are (partially) presented in this thesis. Denise lives together with Bob van der Ruijt and their three cats, Smokey, Scooby and Silvijn. In her leisure time, she works at a children's farm called De Wieringenberg. Currently, she works as a post-doctoral fellow at the faculty of Social Sciences at the University of Utrecht. 